Publ. RIMS, Kryoto Univ.

23 (1987), 345-407

\title{
The Poincare Lemma for Variations of Polarized Hodge Structure
}

By

\author{
Masaki KASHIWARA and Takahiro KAWAI*
}

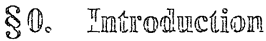

\$0.1. The purpose of this article is to prove the following Conjecture 0.1.1 when $X$ is non-singular $\mathbb{K} a ̈ h l e r$ manifold and $Y^{*}$ is the compiement of a nor. mally crossing hypersurface.

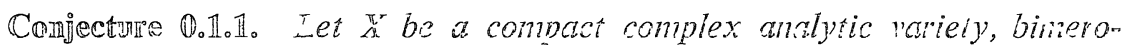
norphic to a compact Kähle" manifold, and let $X^{*}$ be as non-singular Zariski open subset of $X$. Then, for any polarizable varicition of Hodge struciure $\mathbb{H}_{1}$

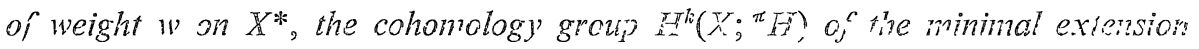
${ }^{\pi} H$ of th admits a canonical pure Erodge structure.

Here the minimal extension ${ }^{\pi} H$ of $H$ is, by definition, a unique perverse sheaf on $X$ such that it is an extension of $H$ and that it does not have either non-zero sub-perverse sheaf or a perverse quotient supported in $X \backslash X^{* *}$.

$\S 0.2_{0}$ Wheru $X^{*}$ is a non-singular Kähler manifolo and $E_{1}$ is a trivial variation, the above conjecture is nothing but the classical result of Hodge. When $x_{-}^{* *}$ is a non-singular Kähler manifold, this is proved by D Deligne (cf. [ $]$ ]) ty the same method of using the theory of harmonic integrals. When $X$ is a curve, this is proved by $S$. Zucker [Z].

The method of our proof follows the idea of $Z$ ucker is that we express the cohomology group of the minimal extension by the $L^{2}-$ cohomology groups and use the theory of harmonic integrals.

§0.3. The shall give an outline of the proof of our main result, i.e., the following

Theorem $\mathbb{Q}_{0.3} \mathbb{1}_{0}$ Let $x^{*}$ be the complement of a nomally crossing hyper-

R.eceived October 29, 1986.

* Research Institute for Miahhematical Sciences, W... oto University, Yyoto 606, Japan 
surface of a compact Kähler manifold. Then, for any polarizable variation of Hodge structure $H, H^{k}\left(X ;{ }^{\pi} H\right)$ has a canonical Hodge structure.

$\$ 0.4$. The first step is to take a Kähler metric $\omega$ on $X^{*}$ whose behavior near the boundary $Y=X \backslash X^{*}$ is as follows:

$$
\omega \sim \sum_{j \leq l}-\sqrt{-1} \partial \bar{\partial} \log \log \left|z_{j}\right|^{2}+\sum_{j>l} \sqrt{-1} d z_{j} d \bar{z}_{j}
$$

for a local coordinate system $\left(z_{1}, \cdots, z_{n}\right)$ of $X$ such that $Y$ is defined by $z_{1} \cdots z_{l}$ $=0$.

$\S \oplus .5$. Let $H=\left(H_{C} ; F(H), \bar{F}(H) ; S\right)$ be a polarized variation of Hodge structure on $X^{*}$. Then, by the definition, $H_{C}$ is a local system on $X^{*}$ and $F(H)$ (resp., $\bar{F}(H)$ ) is a filtration of $\mathcal{O}_{X} \otimes H_{C}$ (resp., $\mathcal{O}_{\bar{X}} \otimes H_{C}$ ). (See $\S 1.3$.) Since the polarization $S$ gives rise to a Hermitian metric on the $C^{\infty}$-bundle associated with $H_{C}$, we can define $L_{(2)}^{k}\left(X^{*} ; H\right)$, the space of $k$-forms with coefficients in $H_{C}$ which are square-integrable. Then this is a Hilbert space.

The $L^{2}$-cohomology group $H_{(2)}^{k}\left(X^{*} ; H\right)$ is, by definition, the quotient space

$$
\left\{u \in L_{(2)}^{k}\left(X^{*} ; H\right) ; d u=0\right\} /\left\{d u ; u \in L_{(2)}^{k-1}\left(X^{*} ; H\right) \quad \text { and } \quad d u \in L_{(2)}^{k}\left(X^{*} ; H\right)\right\} .
$$

We shall prove that $H_{(2)}^{k}\left(X^{*} ; H\right)$ coincides with $H^{k}\left(X ;{ }^{\pi} H\right)$. Once this is proved, then the finite-dimensionality of $H^{k}\left(X ;{ }^{\pi} H\right)$ enables us to employ the theory of harmonic integrals. Hence we find that $H_{(2)}^{k}\left(X^{*} ; H\right)$ is represented by the space of harmonic forms. Then, by decomposing harmonic forms to their $(p, q)$-components, we obtain a Hodge structure on $H_{(2)}^{k}\left(X^{*} ; H\right)$. The last part of the reasoning is almost identical with the classical one (e.g. that in [W]).

Here we call reader's attention to an advantage in using the metric of the form (0.4.1); the metric is complete, and hence we do not need to worry about the contribution from the boundary in performing the integration by parts. This fact diminishes the trouble considerably in handling harmonic integrals.

\$0.6. Thus the problem is to show that $H^{k}\left(X ;{ }^{\pi} H\right)$ is isomorphic to $H_{(2)}^{k}\left(X^{*} ; H\right)$. This problem can be localized as follows:

Let us define the sheaf $\mathcal{L}^{k}(H)$ on $X$ as the sheaf associated with the presheaf: 


$$
X \supset U \mapsto\left\{u \in L_{(2)}^{k}\left(X^{*} \cap U ; H\right) ; d u \in \mathbb{L}_{(2)}^{k+1}\left(X^{*} \cap U ; H\right)\right\} .
$$

Then $\mathcal{L}^{\circ}(H)$ is a complex of sheaves, and we have

$$
H_{(2)}^{k}\left(X^{*} ; H\right)=H^{k}\left(\Gamma\left(X ; \mathcal{L}^{\circ}(H)\right)\right) \text {. }
$$

Here we note another advantage in using the particular $\mathbb{K}$ ähler metric $\omega ; \mathcal{L}^{\circ}(H)$ turns out to be a complex of soft sheaves, and hence

$$
H^{k}\left(\Gamma\left(X ; \mathcal{L}^{\circ}(H)\right)\right)=H^{k}\left(X ; \mathcal{L}^{\circ}(H)\right) \text {. }
$$

Thus we reduce the problem to proving that $\mathcal{L}^{\circ}(H)$ is quasi-isomorphic to ${ }^{\pi} H$; this is a local problem.

\$0.7. In order to prove that $\mathcal{L}^{\circ}(H)$ is quasi-isomorphic to ${ }^{\pi} H$, we need to know, of course, the behavior of the Hermitian metric on the bundle $\mathbb{H}_{C}$ given by the polarization. It is described in terms of the monodromy of $H_{C}$ around $X \backslash X^{*}$.

$\$ 0.8$. Now that the problem is reduced to a local problem, we may assume $X=\Delta^{n}$ and $X^{*}=\Delta^{* n}$, where $\Delta \subset \mathbb{C}$ is the open unit disc and $\Delta^{*}=\Delta \backslash\{0\}$, the punciured disc. Moreover, by the hypothesis of the induction, we may assume that $\mathcal{L}^{\circ}(H) \cong{ }^{\pi} H$ holds on $X \backslash\{0\}$.

Let us now write $X \backslash\{0\} \cong\{t ; 0<t\} \times L$, where $L$ is a "link" and $t$ denotes the radial coordinate. Then $H^{\circ}\left(X ; \mathcal{L}^{\circ}(H)\right)$ is isomorphic to the $L^{2}$-cohomology group $H_{(2)}^{\circ}(X \backslash\{0\} ; H)$.

Now, $H^{k}\left(X \backslash\{0\} ; \mathcal{L}^{\circ}(H)\right)$ is isomorphic to $H_{(2)}^{k}(L ; H)$, which is represented by the space $h^{k}$ of harmonic $k$-forms on $L$. On the other hand, we can show that $H_{(2)}^{\circ}(X \backslash\{0\} ; H)$ is isomorphic to the cohomology group of square-integrable $h=\bigoplus_{k} h^{k}$-valued forms on the $i$-space with respect to the norm

$$
\int\left\|t^{K} h(i)\right\|^{2} d t / t
$$

Here $K$ is an endomorphism of $h$. (See $\S 5.8$.) Then, by the characteristic property of the intersection cohomology groups, it suffices to verify

$$
H_{(2)}^{k}(X \backslash\{0\} ; H) \cong \begin{cases}h^{k}, & \text { for } k<n \\ 0, & \text { for } k \geqq n\end{cases}
$$

The proof of this fact can be reduced to the estimation of the eigenvalues of $K$. The required estimation follows from the purity theorem (Theorem 4.0.1), as we are going to explain below. 
\$0.9. By [S], any variation of Hodge structure is locally described by a nilpoteni orbit. A nilpoten $t$ orbit of weight $w$ is, by definition, $\left(H ; F ; N_{1}, \cdots, N_{l} ; S\right)$, where $\left\{N_{j}\right\}$ is a set of commuling nilpotent endomorphisms of a vector space

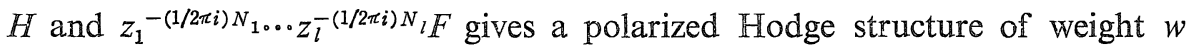
for $0<\left|z_{1}\right|, \cdots,\left|z_{l}\right| \ll 1$ (See Definition 1.2.3.) Then this gives a variation $\mathbb{H}_{\mathbb{C}}$ of polarized Hodge structure on $\Delta^{* n} \cap U$, where $U$ is a neighborhood of the origin.

Now let ${ }^{\pi} H_{C}$ be the minimal extension of $H_{C}$. Then its cohomology group $H^{k}\left({ }^{\pi} H_{C}\right)_{0}$ can be calculated as the cohomology group of the partial Koszul complex

(See §3.)

$$
\Pi\left(N_{1}, \cdots, N_{l}\right): H \rightarrow \bigoplus_{j} \operatorname{Im} N_{j} \rightarrow \bigoplus_{j<k} \operatorname{Im} N_{j} N_{k} \rightarrow \cdots
$$

Since $\Pi\left(N_{1}, \cdots, N_{l}\right)$ can be regarded as a complex of mixed Hodge structures, the purity theorem (Theorem 4.0.1) asserts that the weight of $\mathbb{H}^{k}\left(\mathbb{I}\left(N_{1}\right.\right.$, $\left.\cdots, N_{l}\right)$ ) is $\leqq k+w$. Then the required bound on the eigenvalues of $K$ follows from this fact. (See $\$ 5.13$.)

\$1.10. Theorem 0.3.1 was announced in $[\mathbb{K}-\mathbb{K}, 1]$. E. Cattani, A. Kaplan and W. Schmid obtained similar results independently. ([C- $\mathbb{K}-\mathbb{S}, 2])$

An algebro-geometric construction of the Hodge filtration on $H^{k}\left(X ;{ }^{\pi} H\right)$ is announced in $[K-\mathbb{K}, 2]$. Its details will be published in a forthcoming paper.

§011. As a by-product of Theorem 0.3.1, we obtain the following hard Lefschetz theorem $(\$ 6.3)$ :

Theorem $\mathbb{0}_{0} \mathbb{1 1}_{1} \mathbb{1}_{\text {. }}$ Let $\omega_{0}$ be a Kähler form on $X$ and denote by $\left[\omega_{0}\right]$ its second cohomology class. Then

$$
\left(\left[\omega_{0}\right] \wedge\right)^{k}: H^{n-k}\left(X ;{ }^{\pi} H\right) \rightarrow H^{n+k}\left(X ;{ }^{\pi} H\right)
$$

is an isomorphism (of Hodge structures). Here $n=\operatorname{dim} X$.

\section{$\S 1$. Prelinminaries}

In order to fix the notations, let us review basic notions and results concerning a variation of polarized Hodge structure. Except for some minor modifications, we mainly follow the notations used by Deligne [D], Schmid [S] and Cattani-Kaplan [C-K, 1], [C-K, 2]. 


\section{§1.1. Polarized HIodge Structuries}

Let us first recall the following

Deimintion $1_{0}$ 1.1. Let $\mathfrak{a}$ be an Abelian category. A finite decreasing filtration $F$ of an object $M$ of $\mathfrak{a}$ is, byde finition, a decreasing sequence $\left\{\mathbb{F}^{p}\right\}$ of subobjects of $M$ such that $F^{b}=M$ for $p \ll 0$ and that $F^{p}=0$ ior $p \gg 0$. Its graduation $G r_{F}^{p}$ is, by definition, $F^{p} / \mathbb{F}^{p+1}$.

Remark 1.1.2. (i) By setting $\mathbb{F}_{p}=F^{-p}$, we find an increasing filtration. We use the convention that, if the index $p$ is a subscript (resp., superscript), then $F_{p}$ (resp., $F^{p}$ ) is an increasing (resp., a decreasing) filtration. This convention also applies to $\operatorname{Gr}_{F}^{p}$ and $\mathrm{Gr}_{p}^{F}$.

(ii) If there is no fear of confusion, we usually omit the adjective "finite".

For a filtration $F$, let $F(l)$ be the filtration given by $F_{p}(l)=F_{p+l}$.

Defimition $\mathbb{1}_{0} \mathbb{1}_{0} \mathcal{B}_{0}$ A pair $\left\{F_{1}, F_{2}\right\}$ of niltrations of $\mathbb{N}$ is called $w$-opposed if either one of the following two conditions is satisfied:

$$
\begin{aligned}
& F_{1}^{b} \oplus F_{2}^{q} \rightarrow M \quad \text { for } p+q=w+1 . \\
& \bigoplus_{p+q=w}\left(F_{1}^{b} \cap F_{2}^{q}\right) \stackrel{\rightarrow}{\rightarrow} .
\end{aligned}
$$

For a $\mathbb{C}$-vector space $V$, we denote by $\bar{V}$ the complex conjugate of $V$. Let ${ }^{-}: V \rightarrow \bar{V}$ be the $\mathbb{R}$-isomorphism such that $\overline{a x}=\bar{a} \bar{x}$ for $a \in \mathbb{C}, x \in V$. For $f \in \operatorname{Hom}_{\boldsymbol{C}}\left(V_{1}, V_{2}\right)$, we denote by $\bar{f} \in \operatorname{Hom}_{\boldsymbol{C}}\left(\bar{V}_{1}, \bar{V}_{2}\right)$ its complex conjugate.

Definnition $\mathbb{1}_{0} \mathbb{R}_{\text {.A. }}$. For $w$ in $\mathbb{Z}$, a Hodge siructure $I$ of weight $w$ consists, by definition, of data $\left(H_{C}, F(H), \bar{F}(H)\right)$, where $\mathbb{H}_{C}$ is a finite-dimensional $\mathbb{C}^{\prime}$ vector space, $F(H)$ and $\bar{F}(H)$ are w-opposed filtrations of $H_{\mathbb{C}}$.

For a Hodge structure $H$, its complex conjugate $\bar{H}$ is naturaily defined by $\bar{H}_{C}=\bar{H}_{C}, F(\bar{H})=\overline{\bar{F}(H)}$ and $\bar{F}(\bar{H})=\overline{F(H)}$.

Remark 1.1.5. Set $H^{p, q}(H)=\bar{F}^{p}(H) \cap \bar{F}^{q}(H)$ for $p+q=w$. Then we find

$$
\bigoplus_{p+q=w} H^{p, q}(H) \cong H_{C}
$$

Furthermore $\overline{\mathbb{H}^{p, q}(H)}=H^{q, p}(\bar{H})$ hold's.

Definition $\pi_{0} \mathbb{1}$.6. The Weil operator $\mathrm{C}=\mathrm{C}(H)$ is, by definition, the automorphism of $H_{C}$ given by

$$
\left.\mathrm{C}\right|_{H^{p, q}\left(H_{C}\right)}=i^{p-q}
$$


Remark 1.1.7. It is clear that $\overline{\mathrm{C}(H)}=\mathrm{C}(\bar{H})$ holds.

Defimition 1 .1.8. A polarization of a Hodge structure $H$ is a bilinear homomorphism $S: H_{C} \otimes \bar{H}_{C} \rightarrow \mathbb{C}$ satisfying

(i) $S\left(F^{p}(H), F^{q}(\bar{H})\right)=S\left(\bar{F}^{p}(H), \bar{F}^{q}(\bar{H})\right)=0$ for $p+q>w$,

(ii) $S(\mathbb{C}(H) x, \bar{y})$ is a positive definite Hermitian form on $H_{C}$.

We then immediately find the following

Proposition 1.1.9. (i) $\overline{S(x, \bar{y})}=(-1)^{w} S(y, x)$ for $x, y \in H_{C^{\circ}}$

(ii) $S\left(\mathrm{C}(H) x, \overline{\mathrm{C}(H) y)}=S(x, y)\right.$ for $x, y \in H_{C}$.

(iii) $H_{C}=\bigoplus_{p+q=w} H^{p, q}(H)$ is an orthogonal decomposiiion.

(iv) If $S$ is a polarization of $H$, then $\bar{S}: \bar{H}_{C} \otimes H_{C} \rightarrow \mathbb{C}$, given by $\bar{S}(x, y)=\overline{S(\bar{x}, \bar{y})}$ $=(-1)^{w} S(y, x)$, is a polarization for $\bar{H}$.

Remark 1.1.10. (i) If $H=\bar{H}$, then these definitions coincides with the usual one (e.g. [D] with $A=\mathbb{R}$ ).

(ii) If $H$ is a Hodge structure of weight $w$, then $H^{\prime}=H \oplus \bar{H}$ is a Hodge structure of weight $w$ with $\bar{H}^{\prime}=H^{\prime}$. Therefore we can apply the results of $[\mathrm{S}]$, $[\mathrm{C}-\mathbb{K}, 2]$, [K], etc. in this setting.

Definition 1.1.11. Let $W, F, F$ be three filtrations of $H_{C}$. We say that $(W, F, \bar{F})$ is a mixed Hodge structure of weight $w$ if $\left(F\left(\mathrm{Gr}_{k}^{W}\right), \bar{F}\left(\mathrm{Gr}_{k}^{W}\right)\right)$ is $(w+k)$ opposed for any $k$. If $w=0$, we simply call it a mixed Hodge structure.

As proved in [D], mixed Hodge structures form an Abelian category and $W_{k}, \mathrm{Gr}_{k}^{W}, \mathrm{Gr}_{F}^{p}, \mathrm{Gr}_{F}^{p}$, etc. are exact functors from the category of mixed Hodge structures.

Definition 1.1.12。 A mixed Hodge structure is said to be split if

$$
H=\bigoplus_{p, q} F^{p}(H) \cap \bar{F}^{q}(H) \cap W_{p+q}(H)
$$

holds.

Remark 1.1.13. The condition (1.1.4) is equivalent to saying that $\{F(H)$, $\bar{F}(H), W(H)\}$ is distributive. (See $[\mathrm{K}]$.)

Defimition 1.1.14. For a mixed Hodge structure $H$, we define $H\left(k_{1}, k_{2}\right)$ by $H\left(k_{1}, k_{2}\right)=H_{C}, W_{k}\left(H\left(k_{1}, k_{2}\right)\right)=W_{k+k_{1}+k_{2}}(H), F^{p}\left(H\left(k_{1}\right)\right)=F^{p+k_{1}}(H)$ and $\bar{F}^{p}\left(H\left(k_{1}, k_{2}\right)\right)=\bar{F}^{p+k_{2}}(H)$. We denote $H(n, n)$ by $H(n)$. 


\section{§1.2. The Minodromy Weight Fillirrations æind Nilpocent Oribits}

In later sections we encounter nilpotent endomorphisms defined through monodromy transformations, and they are the starting of our reasoning. So let us prepare some formalities related to nilpotent endomorphisms.

Let $M$ be an object of an Abelian category and $N$ a nilpotent endomorphism of $M$. Then there exists a unique filtration $\mathbb{W}$ of $N$ which satisfies the following conditions (See e.g. [D].)

$$
\begin{gathered}
N W_{k} \subset \mathbb{W}_{k-2} \\
N^{k}: \mathrm{Gr}_{k}^{W} \rightarrow \mathrm{Gr}_{-k}^{W} \quad \text { is an isomorphism for } k \geqq \mathbb{1} .
\end{gathered}
$$

This filtration is called the $N$-filtration or the weight filtration of $N$ and denoted by $\mathbb{W}(N)$. This filtration is given inductively by the foliowing formula:

$$
\begin{aligned}
& W_{k-1}(N)=\left(N^{k}\right)^{-1} W_{-k-1}(N) \text { and } \\
& W_{-k}(N)=N^{k} W_{k}(N) \quad \text { for } \quad k \geqq 1 .
\end{aligned}
$$

For an integer $k \geqq 0$, we denote by $P_{k}(N)$ the kernel of $N^{k+1}: \mathbb{G r}_{k}^{W(N)} \rightarrow$ $\mathrm{Gr}_{-k-2}^{W(N)}$. Then we have

$$
\begin{aligned}
\operatorname{Gr}_{k}^{W(N)} & =\mathbb{P}_{k}(N) \oplus N \mathrm{Gr}_{k+2}^{W(N)} \\
& \cong \bigoplus_{j \geqq 0} \mathbb{P}_{k+2 j}(N)
\end{aligned}
$$

We have also

$$
P_{k}(N) \leftleftarrows \frac{\mathbb{K e r} N^{k+1}}{\operatorname{Ker} N^{k}+\operatorname{Im} N \cap \mathbb{K e r} J^{k+1}} .
$$

In order to introduce the notion of a nilpotent orbit (Definition 1.2.3 below), let us prepare some notations.

Let $w$ be an integer, $H$ a $\mathbb{C}$-vector space, and $h^{p}$ a non-negative integer such that $\operatorname{dim} H=\sum h^{p}$. Let $S$ be a non-degenerate bilinear form $\mathbb{H} \otimes \bar{H} \rightarrow \mathbb{C}$ such that $\overline{S(x, \bar{y})}=(-1)^{w} S(y, \bar{x})$. We then denote by $\mathbb{D}$ the flag manifold $\left\{F ; F\right.$ is a filtration of $H$ such that $\left.\operatorname{dim} \mathbb{G}_{i}^{p}=h^{p}\right\}$. For $F \in \mathbb{D}$, we set $\bar{F}^{p}=$ $\left(F^{w+1-p}\right)^{\perp}$. Here, for a subspace $V$ of $\mathbb{H}, V^{\perp}=\left\{x \in \mathbb{H}^{i} ; S(x, \bar{V})=0\right\}=$ $\{x \in H ; S(V, \bar{x})=0\}$. Let $D$ denote $\{F \in \mathbb{D} ;(F, \bar{F})$ is a Hodge structure of weight $w$ and $S$ gives its polarization\}. Set $G=G L^{\pi}\left(\mathbb{H}^{r}\right)$ and $G_{\mathbb{R}}=\mathbb{U}(\mathbb{S})=$ $\{g \in G ; S(g x, \overline{g y})=S(x, \bar{y})\}$, and let $g$ and $\mathrm{g}_{\mathbb{R}}$ denote their $\mathbb{L}$ ie algebras. Then $D$ and $\check{D}$ are homogeneous spaces of $G_{\mathbb{R}}$ and $G$, respectively (if they are not empty). For $\mathbb{F} \in \mathscr{D}, T_{F} \check{D} \cong \mathfrak{g} / \mathbb{F}^{0}(\mathrm{~g})$, where $\mathbb{F}^{p}(\mathrm{~g})=\left\{a \in \mathrm{g} ; a \mathbb{F}^{k} \subset \mathbb{F}^{k+p}\right.$ for any

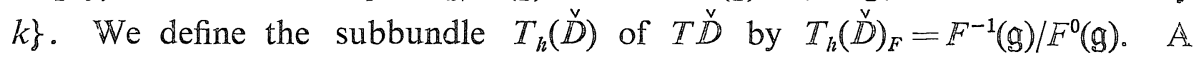


holomorphic map $f: X \rightarrow \mathscr{D}$ from a complex manifold $X$ to $\mathscr{D}$ is called horizontal if $d f(T X) \subset T_{h}(\check{D})$.

We know that (e.g. $[\mathrm{G}-\mathrm{S}],[\mathrm{K}]$ ) there exists a real analytic function $\delta$ on $D$ which satisfies the following conditions:

(i) For any $\alpha \in \mathbb{R},\{x \in D ; \delta(x) \leqq \alpha\}$ is compact:

(ii) For any horizontal holomorphic map $f: X \rightarrow D, \delta \circ f$ is pluri-subharmonic.

The following lemma is due to Cattani-Kaplan [C-K, 2].

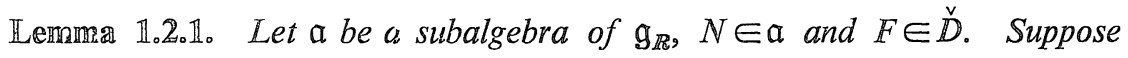
that $A W_{k}(N) \subset W_{k-2}(N)$ for any $A \in \mathfrak{a}$, and that $(W(N), F, \bar{F})$ is a mixed Hodge structure of weight $w$. Then there exists $A \in \mathfrak{g}_{\mathbb{R}}$ such that

(1.2.7) $[A, a]=0$,

(1.2.8) $A W_{k}(N) \subset W_{k-2}(N)$.

The main part of the following proposition is also due to Cattani-Kaplan $[\mathrm{C}-\mathrm{K}, 2]$.

Proposition $\mathbb{1}_{0} 2_{2} 2_{0}$ Let $\mathfrak{a}$ be an Abelian subalgebra of $\mathfrak{g}_{\mathbb{R}}$ consisting of nilpotent elements, $C$ a connected open cone of a and $F \in \check{D}$.

Assume that

(1.2.9) $N F^{p} \subset F^{p-1}$ for $\quad N \in a$.

Then the following conditions are equivalent:

(i) There exists $N_{0} \in a$ such that $e^{i N} F \in D$ for $N \in C+N_{0}$.

(ii) There exists $N_{0} \in$ a such that $e^{i N} F \in D$ for $N \in \mathrm{Ch}(C)+N_{0}$, where $\mathrm{Ch}(\mathrm{C})$ denotes the convex hull of $C$.

(iii) The $N$-filtration $W(N)$ does not depend on $N$ in $C$, and the following two conditions are satisfied for any $N$ in $C$.

(iii a) $(F, \bar{F}, W(N))$ is a mixed Hodge structure of weight $w$.

(iii b) The bilinear form $S\left(\mathrm{Gr}_{k}^{W(N)}\right)$ : $\operatorname{Gr}_{k}^{W(N)} \otimes \overline{\mathrm{Gr}_{k}^{W(N)}} \rightarrow \mathbb{C}$ given by $S\left(x, \overline{N^{k} y}\right)$ endows the Hodge structure on $\mathbb{P}_{k}(N)$ with a polarization for $k \geqq 0$.

(iv) We have (iii) except that we require (iii b) for some $N$ in $C$.

Proof. (i) $\Rightarrow$ (iii) is due to Schmid [S] and Cattani-Kaplan [C-K, 2]. 
(iii) $\Leftrightarrow$ (iv) follows from the following two facts:

(1.2.9) If $W(I N)$ is independent of $N$ and the condition (iii a) is satisfied, then $S\left(G \mathrm{rr}_{k}^{W(N)}\right)$ gives a non-Gegenerate Hermitian form on $\mathbb{P}_{k}(N)$.

(1.2.10) Any member of a continuous and connected family of nom-degenerate Hermitian forms is positive-definite if some member is positivedefinite.

Thus the proof of the proposition is completed if we show (iii) $\Rightarrow$ (ii). Iet us now begin its proof. Set $W=\mathbb{W}(N)$. Ry the preceding lemma, there exists $A \in \mathfrak{S}_{\mathbb{R}}$ which satisfies the following conditions:

$$
A W_{k} \subset W_{k-1} \text {, }
$$

(1.2.12) If we set $\mathbb{F}_{0}=e^{i A} \mathbb{F}$ and $\bar{F}_{0}=e^{-i A} \bar{F}$, then $\left(W, \mathbb{F}_{0}, \bar{F}_{0}\right)$ is split.

Then by [C- $\mathbb{K}, 2]$

$$
e^{i N} \mathbb{F}_{0} \in D \text { for } N \in C \text {. }
$$

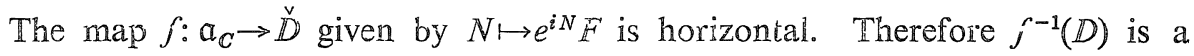
holomorphically convex. Moreover $f^{-1}(D)+i a=f^{-1}(D)$ holds, because $D$ is invariant under the action of $G_{\mathbb{R}}$. Therefore we can apply the celebrated theorem of Bochner: a connected pseudo-convex tube domain is convex. Thus any connected component of $f^{-1}(D)$ is convex.

IJow, let $\mathbb{Y}$ be an element of $\mathbb{g}_{\mathbb{R}}$ which satisies

$$
\left.Y\right|_{\mathbb{F}_{0}^{p} \cap \bar{F}_{0}^{q} \cap W_{p+q-w}}=p+q-w .
$$

Then we find

$$
\begin{gathered}
Y W_{k} \subset W_{k} \\
\left.Y\right|_{G r_{k}^{W}}=k \circ \mathrm{id}, \\
{[Y, N]=-2 N \text { for } N \in \mathbb{Q},} \\
Y \mathbb{F}_{0}^{p} \subset \mathbb{F}_{0}^{p} .
\end{gathered}
$$

We also note

$$
e^{i N} \mathbb{F} \in \mathbb{D} \Leftrightarrow i^{Y} e^{i N} \mathbb{F} \in \mathbb{D} .
$$

On the other hand, a simple calculation shows

$$
\hat{\imath}^{Y} e^{i N} F=e^{i t^{-2} N-i \operatorname{Ad}\left(t^{Y}\right) A} F_{0} .
$$

Writing $A=\sum A_{j}$ with $\left[Y, A_{j}\right]=j A_{j}^{\prime}$, we deduce 


$$
A_{j}=0 \quad \text { for } \quad j \geqq 0
$$

from $A W_{k} \subset W_{k-1}$. Hence we obtain

$$
\operatorname{Ad}\left(t^{Y}\right) A=\sum_{j<0} t^{j} A_{j}
$$

Therefore $\operatorname{Ad}\left(t^{Y}\right) A$ tends to 0 as $t$ tends to $\infty$. Since $e^{i N} F_{0} \in D$, for any compact subset $K$ of $C$ there exists a constant $\alpha_{K}>0$ such that

$$
e^{i N} F \in D \text { if } t^{-2} N \in K \text { and } t \geqq \alpha_{K} .
$$

This shows that $f^{-1}(D) \supset\left\{t ; t \geqq \alpha_{K}^{2}\right\} \circ K$. In particular, $f^{-1}(D) \supset\left\{t N_{0} ; t \geqq 1\right\}$ for some $N_{0} \in C$. Let $C^{\prime}$ be the connected component of $f^{-1}(D) \cap \mathfrak{a}_{\mathbb{R}}$ which contains $\left\{t N_{0} ; t \geqq 1\right\}$. Let us now prove

$$
C^{\prime} \supset C+N_{0}
$$

For $N \in C$, take a connected compact subset $K$ of $C$ which contains both $N$ and $N_{0}$. Then

$$
C^{\prime} \supset t K \text { for } t \geqq \alpha_{K}^{2}
$$

Hence $C^{\prime}$ contains

$$
\frac{1}{t+1}\left(t N+t N_{0}\right)=N+N_{0}-\frac{1}{t+1}\left(N+N_{0}\right)
$$

for $t \geqq \alpha_{K}^{2}$. This means that the closure $\bar{C}^{\prime}$ of $C^{\prime}$ contains $N+N_{0}$. Since $C^{\prime}=$ Int $\bar{C}^{\prime}$, we obtain (1.2.23). This completes the proof of Proposition 1.2.2.

Now we introduce the following

Deimnition 1.2.3. If one of the equivalent conditions in Proposition 1.2.2 is satisfies, we say that $(F, \bar{F} ; C)$ forms a nilpotent orbit of weight $w$. For a finite set $\mathbb{I}$ of mutually commuting nilpotent elements of $\mathfrak{g}_{\mathbb{R}}$, we say that $(F, \bar{F} ; I)$ forms a nilpotent orbit if $(F, \vec{F} ; C(I))$ forms a nilpotent orbit, where

$$
C(I)=\left\{\sum_{N \in I} t_{N} N ; t_{N}>0\right\}
$$

\section{§1.3. Variation of polarized Hodge Structure}

Let $X$ be a complex manifold and $\bar{X}$ the complex conjugate of $X$. A variation of Hodge structure of weight $w$ on $X$ is, by definition, a triplet $\left(H_{C}, F, \bar{F}\right)$ of a locally constant $\mathbb{C}_{X}$-module $H_{C}$ of finite rank and a finite filtration $F(H)$ (resp., $\bar{F}(H)$ ) of $\mathcal{O}_{X}(H)=\mathcal{O}_{X} \bigotimes_{C} H_{C}$ (resp., $\mathcal{O}_{\bar{X}}(H)=\mathcal{O}_{\bar{X}} \bigotimes_{C} H_{C}$ ) 
by vector subbundles which satisfy the following conditions:

(1.3.1) At each point $x$ in $X, H(x)=\left(H_{C, x}, F(H)(x), \bar{F}(H)(x)\right)$ is a Hodge structure of weight $w$.

(1.3.2) For any holomorphic vector field $v, v F^{p}(H) \subset \mathbb{F}^{p-1}(\mathbb{H})$ and $\bar{v} \bar{F}^{p}(H) \subset$ $\bar{F}^{p-1}(H)$ hold.

A variation of Hodge structure is said to be polarized, if a bilinear homomorphism $S: H_{C} \otimes \bar{H}_{C} \rightarrow C_{X}$ is given so that $S_{x}$ polarizes $H(x)$ at each point $x$ in $X$.

\section{§2. Vamishing Cycle Theorem}

§2.0. Let $X^{*}$ be a punctured disc and let $H$ be a variation of Hodge structure of weight $w$ with a unipotent monodromy. Let $\psi$ be the near-by cycle. Then Schmid's theorem says that $\psi$ carries a mixed Hodge structure, called the limit mixed Hodge structure in literature. On the other hand, the vanishing cycle $\varphi$ in this setting is $\operatorname{Im} N$ and can: $\psi \rightarrow \varphi$ is given by $N$ and var: $\varphi \rightarrow \psi$ is given by the inclusion map. (cf. [D]) The purpose of this section is to prove that the vanishing cycle also carries a mixed Hodge structure. (Theorem 2.1.5 below.)

§2.1. Lê $N$ be a nilpotent endomorphism of an object $M$ of an Abelian category. Set $M_{0}=\operatorname{Im} N, N_{0}=\left.N\right|_{M_{0}} \in \mathbb{E}$ nd $\left(\mathbb{M}_{0}\right)$. Then we have the following proposition.

Proposition 2.1.1. (i) $W_{k}\left(N_{0}\right)=I V W_{k+1}(N)=M_{0} \cap W_{k-1}(N)$.

(ii) $M \rightarrow M_{0} \rightarrow M$ induces a surjective morphism $\mathrm{Gr}_{k+1}^{W(N)} \rightarrow \mathrm{Gr}_{k}^{W\left(N_{0}\right)}$ and an injective morphism $\mathrm{Gr}_{k}^{W\left(N_{0}\right)} \rightarrow \mathrm{Gr}_{k-1}^{W(N)}$ so that

$$
\operatorname{Gr}_{k}^{W(N 0)} \cong \operatorname{Im}\left(N: \operatorname{Gr}_{k+1}^{W(N)} \rightarrow \operatorname{Gr}_{k-1}^{W(N)}\right) \cong\left\{\begin{array}{lll}
\operatorname{Gr}_{k+1}^{W(N)} & \text { for } & k \geqq 0 \\
\operatorname{Gr}_{k-1}^{W(N)} & \text { for } & k \leqq 0
\end{array}\right.
$$

(iii) $P_{k}\left(N_{0}\right) \approx P_{k+1}(N)$ for $k \geqq 0$.

Since the proof of this proposition is elementary, we leave it to the reader.

Let $w$ be an integer. Let $H^{r}$ be a finite dimensional $\mathbb{C}$-vector space and $S$ a non degenerate bilinear form $H \otimes \bar{H} \rightarrow \mathbb{C}$ such that $\overline{S(x, \bar{y})}=(-1)^{w} S(y, \bar{x})$. Let $N$ be a nilpotent element of $\mathfrak{\imath}(S ; H)$, the Lie algebra of $U(S)=\{g \in G L(H)$; $S(g x, \overline{g y})=S(x, \bar{y})\}$. Let $H_{0}$ (resp., $\left.N_{0}\right)$ dencte $\operatorname{Im} N$ (resp., $\left.\left.N\right|_{\operatorname{lm} N}\right)$ and define a bilinear form $S_{H_{0}}: H_{0} \otimes \bar{H}_{0} \rightarrow \mathbb{C}$ as follows:

$$
S_{H_{0}}(N x, \overline{N y}) \underset{\text { def }}{=} S(x, \overline{N y})=-S(N x, \bar{y}) \text { for } x, y \in H
$$


It is clear that $S_{H_{0}}$ is a well-defined bilinear form on $H_{0} \otimes \bar{H}_{0}$. Let us list up some elementary properties of $H_{0}$ and $S_{H_{0}}$.

Lemmma 2.1.2。 (i) $\overline{S_{H_{0}}(x, \bar{y})}=(-1)^{w+1} S_{H_{0}}(y, \bar{x})$ holds for $x, y \in H_{0}$.

(ii) $S_{H_{0}}$ is non-degenerate.

(iii) If $[A, N]=2 k N$ and $A$ is in $\mathfrak{u}(\mathbb{S} ; H)$, then $\left.(A-k)\right|_{H_{0}}$ belongs to $\mathfrak{u}\left(S_{0} ; H_{0}\right)$.

(iv) $N: \mathrm{Gr}_{k+1}^{W(N)} \rightarrow \mathrm{Gr}_{k}^{W\left(N_{0}\right)}$ is isometric for $k \geqq 0$, that is, $S\left(x, \bar{N}^{k+1} \bar{y}\right)=$ $S_{H_{0}}\left(N x, \bar{N}^{k}(\overline{N y})\right)$ holds for $x, y \in W_{k+1}(N)$.

The proof of this lemma is again a straightforward one, and it is omitted here.

The following proposition is an immediate consequence of Lemma 2.1.2, (iv).

Proposition 2.1.3. If $(\mathbb{S} ; F, \bar{F} ; N)$ forms a nilpotent orbit of weight $w$, $\left(S_{H_{0}} ; N F, N \bar{F} ; H_{0} ; N_{0}\right)$ forms a nilpotent orbit of weight $w+1$.

Remark 2.1.4. The coincidence of $N F^{p}$ with $F^{p-1} \cap H_{0}$ follows from the fact that any morphism of mixed Hodge structure is strict with respect to the Hodge filtration.

We are now ready to state the vanishing cycle theorem:

Theorem 2.1.5. (Væmishng Cycle Theorem) Suppose that $\{S ; F, \bar{F}$; $\left.N_{1}, \cdots, N_{l}\right\}$ forms a nilpotent orbit of weight $w$. Set $H_{1}=\operatorname{Im} N_{1}$ and let $S_{H_{1}}$ : $H_{1} \otimes \bar{H}_{1} \rightarrow \mathbb{C}$ be given by $S_{H_{1}}\left(N_{1} x, \overline{N_{1} y}\right)=S\left(x, \overline{N_{1} y}\right)$. Then we have

(i) $\left\{S_{H_{1}} ; N_{1} F, N_{1} \bar{F} ;\left.N_{1}\right|_{H_{1}}, \cdots,\left.N_{l}\right|_{H_{1}}\right\}$ forms a nilpotent orbit of weight $(w+1)$.

(ii) $W_{k}\left(\left.N_{1}\right|_{H_{1}}, \cdots,\left.N_{b}\right|_{H_{1}}\right)=N_{1} W_{k+1}\left(N_{1}, \cdots, N_{l}\right)=H_{1} \cap W_{k-1}\left(N_{1}, \cdots, N_{l}\right)$.

Before starting the proof of this theorem, we state and prove the following corollary.

Corollary 2.1. 6 . Suppose that $\left\{\mathbb{F}, \bar{F} ; N_{1}, \cdots, N_{l}\right\}$ forms a nilpotent orbit of weight $w$, and set $W=W\left(N_{1}, \cdots, N_{l}\right)$. Then, for any $N_{0}^{\prime}, N_{1}^{\prime}, \cdots, N_{k}^{\prime} \in$ $\mathbb{C}\left(N_{1}, \cdots, N_{l}\right)$, we have the following:

(i) $N_{1}^{\prime} \cdots N_{k}^{\prime}: \mathrm{Gr}_{k}^{W} \rightarrow \mathrm{Gr}_{-k}^{W}$ is an isomorphism for $k \geqq 1$.

(ii) $S\left(\mathbb{C} x, \overline{\left.N_{1}^{\prime} \cdots N_{k}^{\prime} x\right)}\right.$ is a positive definite Hermitian form on $\operatorname{Ker}\left(N_{0}^{\prime} \cdots N_{k}^{\prime}\right.$ : $\mathrm{Gr}_{k}^{W} \rightarrow \mathrm{Gr}_{-k-2}^{W}$ ).

Proof. We prove (i) by the induction on $k$. If $k=1$, this is evident. 
Assume $k \geqq 2$. Set $H_{0}=\mathbb{I m} N_{1}^{\prime}$ and $\mathbb{V}^{\prime} T^{\prime}=\mathbb{W}\left(\left.N_{1}\right|_{H_{0}}, \cdots,\left.N_{l}\right|_{H_{0}}\right)=\mathbb{P}\left(\left.N_{1}^{\prime}\right|_{H_{0}}\right)$. Then we have the following commutative diagram:

$$
\begin{aligned}
& \mathrm{Gr}_{k}^{W} \stackrel{N_{1}^{\prime} \cdots N_{k}^{\prime}}{\longrightarrow} \mathrm{Gr}_{-k}^{W} \stackrel{N_{0}^{\prime}}{\longrightarrow} \mathrm{Gr}_{-k-2}^{W} \\
& l \downarrow \quad l \uparrow \quad l \uparrow \\
& \mathrm{Gr}_{k-1}^{W W^{\prime}} \underset{N_{2}^{\prime} \cdots N_{k}^{\prime}}{\longrightarrow} \operatorname{Gr}_{1-k}^{W^{\prime}} \underset{N_{0}^{\prime}}{\longrightarrow} \operatorname{Gr}_{-1-k}^{\left[W^{\prime}\right.} .
\end{aligned}
$$

The left bottom arrow being an isomorphim by these induction hypothesis, the left upper arrow is also an isomorphism. The claim (ii) is also an immediate consequence of the diagram (2.1.2).

In connection with this corollary we propose the following

Conjectrne 2.1.7. Let $\left\{F, \bar{F} ; N_{1}, \cdots, N_{l}\right\}$ be a nilpotent orbit of weight $w$ and let $k$ be a positive integer. Lei $f\left(x_{1}, \cdots, x_{l}\right)$ be a homogeneous polynomial of degree k. Suppose that $f^{-1}(0) \cap \mathbb{R}_{+}^{l}=\{0\}$. Then $f\left(N_{1}, \cdots, N_{l}\right): \mathbb{G r}_{k}^{W\left(N_{1}, \cdots, N_{l}\right) \rightarrow}$ $\mathrm{Gr}_{-k}^{\mathrm{W}\left(N_{1}, \cdots, N_{l}\right)}$ is an isomorphism.

The corollary says that this conjecture is true if $f$ is a product of real linear functions.

Now we start the proof of the vanishing cycle theorem by proving it in the following special case.

Lemma 2.1.3. Suppose that $\left\{F, \bar{F} ; N, N^{\prime}\right\}$ forms a nilpotent orbit of weight w. Suppose fuither that $\left\{F, \bar{F} ; W\left(N, N^{\prime}\right)\right\}$ is split. Denoie $\operatorname{Im} N$ by $H_{0}$. Then we find:

(i) There exists a strictly positive number $c$ such that $\left\{N F ;\left.N\right|_{H_{0}}\right.$, $\left.\left.\left(N+c N^{\prime}\right)\right|_{H_{0}}\right\}$ forms a nilpotent orbit of weight $w+1$.

(ii) $W_{k}\left(\left.\left(N+c N^{\prime}\right)\right|_{H_{0}}\right)=N W_{k+1}\left(N, N^{\prime}\right)=H_{0} \cap W_{k-1}\left(N, N^{\prime}\right)$ holds for any sufficiently small number $c>0$.

Proof. (i) Since $\left(F, W\left(N, N^{\prime}\right)\right)$ is split, the assumption implies that $\left(e^{i N^{\prime}} F ; N\right)$ forms a nilpotent orbit of weight $w$. Hence it follows from Proposition 2.1.3 that $\left(e^{i N^{\prime}} N F ; N_{0}\right)$ forms a nilpotent orbit of weight $w+1$. Therefore Proposition 1.2.2 implies that

$$
e^{i N^{\prime}+i t N} N \mathbb{F} \in D
$$

holds for $t>c^{-1}$, where $c$ is a positive constant. Now, let us choose $Y$ in $\operatorname{It}\left(S: H^{\prime}\right)$ such that $[Y, N]=-2 N,\left[Y, I^{\prime}\right]=-2 N^{\prime}$ and $Y F^{p} \subset F^{p}$ hold. Then $Y+\left.1\right|_{H_{0}} \in \mathfrak{l}\left(S_{H_{0}} ; H_{0}\right)$. Then, for any strictly positive number $a,(2.1 .3) \mathrm{im}-$ 
plies

$$
a^{Y+1} e^{i N^{\prime}+i t N} N F=e^{i a^{-2} N^{\prime}+i t a^{-2} N} N F \in D .
$$

This means that $\left(N F ;\left.N\right|_{H_{0}},\left.\left(N+c N^{\prime}\right)\right|_{H_{0}}\right)$ forms a nilpotent orbit.

(ii) This follows from (i) and the following result of Cattani-Kaplan [C-K, 2].

Proposition 2.1.9. Let $N$ be a nilpotent endomorphism of $H$ and let $F, \bar{F}_{\text {, }}$ $W$ be three filtrations of $H$. Assume the following conditions:

(2.1.5) $(W, F, \bar{F})$ is a split mixed Hodge structure of weight $w$.

(2.1.6) $(W(N), F, \bar{F})$ is a mixed Hodge structure of weight $w$.

(2.1.7) $N F^{p} \subset F^{p-1}, \quad N \bar{F}^{p} \subset \bar{F}^{p-1}, \quad N W_{k} \subset W_{k-2}$.

Then $W=W(N)$.

Remark 2.1.10. Cattani-Kaplan [C-K, 2] added another condition which is superfluous; their proof works well without it.

Let us now embark on the proof of Theorem 2.1.5.

Set $W=W\left(N_{1}, \cdots, N_{l}\right)$. By Lemma 1.2 .1 , we can choose $A$ in $\mathfrak{t}(S ; H)$ so that it satisfies the following;

$$
\begin{gathered}
A W_{k} \subset W_{k-1}, \\
{\left[A, N_{k}\right]=0 \quad(j=1, \cdots, l),} \\
\left(W ; e^{i A} F, e^{-i A} \bar{F}\right) \quad \text { is split. }
\end{gathered}
$$

If we can prove Theorem 2.1 .5 for $\left(W ; e^{i A} F, e^{-i A} \bar{F}\right)$, then the theorem holds for $(W ; F, \bar{F})$. Hence we may assume from the first that $(W ; F, \bar{F})$ is split. We may further assume $l=2$. In fact, if the theorem holds for $l=2$, then, for $N$ in $C\left(N_{2}, \cdots, N_{l}\right),\left\{N_{1} F ;\left.N_{1}\right|_{H_{1}},\left.N\right|_{H_{1}}\right\}$ forms a nilpotent orbit.

Now, we prove the theorem by the induction on $\operatorname{dim} H$.

Define the set $A$ by

$\left\{t>0 ;\left\{N_{1} F ;\left.N_{1}\right|_{H_{1}},\left.\left(N_{1}+t N_{2}\right)\right|_{H_{1}}\right\}\right.$ forms a nilpotent orbit $\}$.

The preceding lemma guarantees that $A$ is not void. The theorem is equivalent to $A=\{t ; t>0\}$. Suppose $A \neq\{t ; t>0\}$. Then $A=(0, c]$ for some $c>0$. Let $N_{0}$ denote $N_{1}+c N_{2}$. If we can prove

$$
W_{k}\left(\left.N_{0}\right|_{H_{1}}\right)=N_{1} W_{k+1}
$$

holds for every $k$, then Proposition 1.2 .2 tells us that $c+\varepsilon(0<\varepsilon \ll 1)$ belongs 
to $A$. This is a contradiction. Thus the proof of the theorem is reduced to the proof of (2.1.11). In order to prove (2.1.11), it suffices to prove that

$$
N_{0}^{k}: \mathrm{Gr}_{k+1}^{N_{1} W} \rightarrow \mathrm{Gr}_{-k+1}^{N_{1} W}
$$

is an isomorphism for each $k \geqq 1$.

Let us now define a filtration $\{W(k)\}$ of $H_{k}=\operatorname{Im} N_{1}^{k}$ by the following:

$$
W_{l}(k)=N_{1}^{k} W_{l+k}\left(N_{1}, N_{2}\right)=H_{k} \cap W_{l-k}\left(N_{1}, N_{2}\right) .
$$

The last identity follows from the fact that $N_{1}^{k}$ is a morphism of mixed Hodge structure, which is strict with respect to the weight filtration. Note that

$$
W(k)=W\left(\left.N\right|_{H_{k}}\right) \quad \text { for any } \quad N \in \mathbb{C}\left(N_{1}, N_{0}\right)
$$

holds by the hypothesis of the induction.

In order to prove (2.1.12), let us consider the following statement $A(j, a, k)$ :

$$
\begin{aligned}
& A(j, a, k): N_{0}^{j} N^{\prime a}: \mathbb{G r}_{j+a}^{W(k)} \rightarrow \operatorname{Gr}_{-j-a}^{\mathbb{W}(k)} \text { is bijective, } \\
& \text { where } N^{\prime}=N_{0}+\varepsilon N_{1}(0<\varepsilon \ll 1) .
\end{aligned}
$$

Let us fix $\varepsilon(>0)$ sufficiently small so that $A(j, a, 0)$ may hold. Since $W(k)=W\left(\left.N^{\prime}\right|_{H_{k}}\right)$ for any $k, A(0, a, k)$ is also true.

Next let us prove

$$
\begin{gathered}
A(j+1, a, k+1)+A(j+1, a, k-1)+A(j-1, a+1, k) \Rightarrow A(j, a, k) \\
\text { for } j, k \geqq 1 \text { and } a \geqq 0 .
\end{gathered}
$$

Once this implication is proved, the induction proceeds and we obtain $A(j, a, k)$ for every $j, a, k \geqq 0$, in particular, $A(j, 0,1)$. This is what we wanted to prove.

Now, let us return to the proof of (2.1.16). In what follows, $\mathbb{C}$ denotes the Weil operator, as usual. Take $N$ arbitrarily from $C\left(N_{1}, N_{0}\right)$. $\mathbb{B} y$ the induction on $\operatorname{dim} H$, we can employ the result in Corollary 2.1.6. Therefore we have

$$
\operatorname{Gr}_{a+j}^{W(k)} \stackrel{N^{\prime a} N^{j}}{\sim} \operatorname{Gr}_{-a-j}^{W(k)}
$$

and $S_{H_{k}}\left(\mathrm{C} x, \overline{N^{\prime a} N^{j} x}\right)$ is positive definite in $x \in \mathbb{K} \operatorname{er}\left(G_{a+j}^{W(k)} \stackrel{N^{\prime a} N^{j+1}}{\longrightarrow} \mathrm{Gr}_{-a-j-2}^{W(k)}\right)$. If we assume $A(j+2, a, k)$, then $\operatorname{Gr}_{a+j}^{[V(k)} \stackrel{N^{\prime a} N_{0}^{j+1}}{\longrightarrow} \operatorname{Gr}_{-a-J-2}^{W(k)}$ is surjective. Hence 
$\operatorname{Ker}\left(\mathrm{Gr}_{a+j}^{W(k)} \stackrel{N^{\prime a} N^{j+1}}{\longrightarrow} \mathrm{Gr}_{-a-j-2}^{W(k)}\right)$ varies continuously on a neighborhood of $N=N_{0}$. Hence by letting $N$ tend to the boundary point $N_{0}$, we find the following:

(2.1.19) If $A(j+2, a, k)$ is true, then $S_{H_{k}}\left(\mathrm{C} x, \overline{N^{\prime a} N_{0}^{j} x}\right)$ is positive semi-definite on $K_{a, j}^{k}=\operatorname{Ker}\left(\mathrm{Gr}_{a+j}^{W(k)} \stackrel{N^{\prime a} N_{0}^{j+1}}{\longrightarrow} \operatorname{Gr}_{-a-j-2}^{W(k)}\right)$.

Furthermore it follows from $A(j+2, a, k)$ that

(2.1.20) If $x \in K_{a, j}^{k}$ satisfies $S_{H_{k}}\left(\mathrm{C} x, \overline{N^{\prime a} N_{0}^{j} x}\right)=0$, then $N^{\prime a} N_{0}^{j} x=0$ 。

To see this, let us first note by $(2.1 .19)$

$$
\mathrm{S}_{H_{k}}\left(\mathrm{C} K_{a, j}^{k}, \overline{N^{\prime a} N_{0}^{j} x}\right)=0 .
$$

Since $\operatorname{Gr}_{j+a}^{W(k)}=N_{0} \mathrm{Gr}_{j+a+2}^{W(k)}+K_{a, j}^{k}$ and $S_{H_{k}}\left(N_{0} \mathrm{Gr}_{j+a+2}^{W(k)}, \overline{N^{\prime a} N_{0}^{j} K_{a, j}^{k}}\right)=0$, we have $S_{H_{k}}\left(\mathrm{Gr}_{j+a}^{W(k)}, \overline{N^{\prime a} N_{0}^{j} x}\right)=0$. Since $S_{H_{k}}$ gives a perfect pairing between $\mathrm{Gr}_{j+a}^{W(k)}$ and $\overline{\mathrm{Gr}_{-j-a}^{W(k)}}$. We find $N^{\prime a} N_{0}^{j} x=0$.

Now let us show that, for $k, j \geqq 1$,

(2.1.22) $N_{0}^{j} N^{\prime a}: \mathrm{Gr}_{j+a}^{W(k)} \rightarrow \mathrm{Gr}_{-j-a}^{W(k)}$ is injective under $A(j+1, a, k+1)$, $A(j+1, a, k-1)$ and $A(j-1, a+1, k)$.

Suppose $N_{0}^{j} N^{\prime a} x=0$ holds for $x$ in $\operatorname{Gr}_{j+a}^{W(k)}$. Let us consider the following diagrams:

and
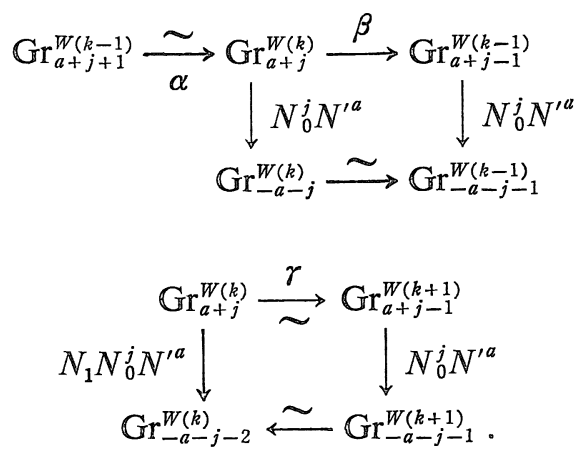

Then $A(j+1, a, k-1)$ implies

$$
S_{H_{k-1}}\left(\mathbb{C} \beta(x), \overline{N_{0}^{j-1} N^{\prime a} \beta(x)}\right) \geqq 0,
$$

and $A(j+1, a, k+1)$ implies

$$
0 \leqq S_{H_{k+1}}\left(\mathrm{Cr}(x), \overline{\left.N_{0}^{j-1} N^{\prime a} \gamma(x)\right)}=S_{H_{k}}\left(\mathrm{C} x, \overline{N_{0}^{j-1} N^{\prime a} N_{1} x}\right)\right.
$$


Now take $y \in \operatorname{Gr}_{a+j+1}^{W(k-1)}$ such that $x=\alpha(y)$. Then $\beta(x)=N_{1} y$ implies

$$
\begin{aligned}
& S_{H_{k-1}}\left(\mathbb{C} \beta(x), \overline{\left.N_{0}^{j-1} N^{\prime a} \beta(x)\right)}=\mathbb{S}_{H_{k-1}}\left(\mathbb{C} N_{1} y, \overline{\left.N_{0}^{j-1} N^{\prime a} N_{1} y\right)}\right.\right. \\
& \quad=-S_{H_{k-1}}\left(\mathbb{C} y, \overline{\left.N_{0}^{j-1} N^{\prime a} N_{1}^{2} y\right)}\right.
\end{aligned}
$$

and

$$
\mathfrak{S}_{H_{k}}\left(\mathbb{C} x, \overline{N_{0}^{j-1} N^{\prime a} N_{1} x}\right)=S_{H_{k-1}}\left(\mathbb{C} y, \overline{\left.N_{0}^{j-1} N^{\prime a} N_{1}^{2} y\right)},\right.
$$

Therefore we find

$$
S_{H_{k-1}}\left(\mathbb{C} \beta(x), \overline{\left.N_{0}^{j-1} N^{\prime a} \beta(x)\right)}=\mathbb{C} .\right.
$$

Hence (2.1.20) implies $N_{0}^{j-1} N^{\prime a} \beta(x)=0$. Then we obtain $N_{0}^{j-1} N^{\prime a} x=0$. Therefore $A(j-1, a+1, k)$ entails $x=0$. This finishes the proof of (2.1.22). Since $\operatorname{dim} \mathrm{Gr}_{j+a}^{W(k)}=\operatorname{dim} \mathrm{Gr}_{-j-a}^{W(k)}$, this means that $N_{0}^{j} N^{\prime a}$ is an isomorphism, that is, $A(j, a, k)$ is verified. Thus we have completed the prooi of Theorem 2.1.5.

\section{§3. The Miminmal Extension amd the Partial [Eoszon Complex}

\$3.0. To understand the meaning of the purity theorem in the subsequent section, we prepare an algebraic result which gives a concrete description of the minimal extension ${ }^{\pi} H$ of a local system $H$.

§3.1. Let $X$ be a complex manifold. We denote by $\mathscr{D}_{X}$ the sheaf of linear differential operators on $X$. Iet $Y$ be a closed analytic subset of $X$ and let $X^{*}$ be $X \backslash Y$ and $j: X^{*} \hookrightarrow X$ the open embedding. Let $\mathscr{M}$ be a regular holono-

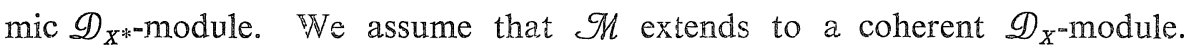
This assumption is equivalent to saying that $j_{!} \mathcal{H}^{k}\left(\mathbb{D} \mathbb{R}_{X^{-1}}(\mathscr{M})\right)$ is constructible for any $k$. Here $\mathbb{D R}=\mathbb{R}$ Hom $(\mathcal{O}$,$) . Then there exists a regular holonomic$ $\mathscr{D}_{X}$-module $\pi \mathscr{H}$ such that

(3.1.1) $\pi \mathscr{M}_{X} \simeq \mathscr{M}$

(3.1.2) $\pi \mathscr{H}$ does not have either non-zero coherent quotient or non-zero coherent submodule with support in $Y$.

Such a $\mathscr{D}_{X}$-module ${ }^{\pi} \mathscr{M}$ is unique up to an isomorphism. We call it the minimal extension of $\mathcal{H}$.

§3.2. Let $f: X^{\prime} \rightarrow X$ be a finite morphism of complex manifolds. Set $Y^{\prime}=f^{-1}(Y)$ and $X^{* \prime}=X^{\prime} \backslash Y^{\prime}=f^{-1}\left(Y^{*}\right)$. For a regular holonomic $\mathscr{D}_{X^{\prime \prime m}}$ module $\mathscr{H}^{\prime}$ extendable to $X^{\prime}$, let $\pi \mathscr{H}^{\prime}$ be the minimal extension of $\mathscr{H}^{\prime}$. Set $\mathscr{M}=\int_{f^{\prime \prime}} \mathscr{M}^{\prime}$, where $f^{* \prime}$ is $Y^{* \prime} \rightarrow X^{*}$. Then we have $\pi \mathscr{M}=\int_{f} \mathscr{M}^{\prime}$. This follows from the following facts: 
(3.2.1) $\int_{f}$ is a faithful exact functor from the category of coherent $\mathscr{D}_{X^{\prime}}$ modules to that of coherent $\mathscr{D}_{X}$-modules. $\int_{f} \mathscr{M}^{*}=\left(\int_{f} \mathscr{M}\right)^{*}$ for any holonomic $\mathscr{D}_{X^{\prime}}$-module $\mathscr{M}$, where * means the adjoint system.

(3.2.3) $\mathscr{H}_{Y}^{k}\left(\int_{f} \mathscr{M}^{\prime}\right)=\int_{f} \mathscr{H}_{Y}^{k}\left(\mathscr{H}^{\prime}\right)$ for any $k$ and any coherent $\mathscr{D}_{X^{\prime}}$-module $\mathscr{M}^{\prime}$.

§3.3. Let $X$ be a non-singular algebraic variety defined over $\mathbb{C}$. Let $\bar{X}$ be the compactification of $X$, i.e., a non-singular proper algebraic variety which contains $X$ as a Zariski open subset and let $\iota: X \hookrightarrow \bar{X}$ be the open embedding. Let $X_{a n}$ be the complex analytic variety associated with $X$. A coherent $\mathscr{D}_{X^{-}}$ module $\mathscr{M}$ is called regular holonomic if $\mathscr{D}_{\bar{X}_{a n}} \otimes_{\bar{X}} c_{*} \mathscr{M}$ is a regular holonomic $\mathscr{D}_{\bar{X}_{\text {AF }}}$-module. Then we have

$$
H^{k}\left(X_{a n}: \mathrm{DR}_{X_{a n}}\left(\mathscr{M}_{a n}\right)\right) \simeq H^{k}\left(X ; \mathbb{D R}_{X}(\mathscr{M})\right) .
$$

Here $\mathrm{DR}_{X}(\mathscr{M})$ denotes the algebraic de $\mathrm{Rham}$ complex and $\mathscr{M}_{a n}$ is $\mathcal{O}_{X_{a n}} \otimes \mathcal{M}$. In fact, assuming ‘ affine, $H^{k}\left(\bar{X}_{a n}, \mathrm{DR}_{\bar{X}_{a n}}\left(\left(\iota_{*} \mathscr{M}\right)_{a n}\right)\right) \underset{\rightarrow}{\rightarrow} H^{k}\left(X_{a n} ; \mathrm{DR}_{X_{a n}}\left(\mathscr{M}_{a n}\right)\right)$ follows from the regularity of $\mathscr{M}$ and $H^{k}\left(\bar{X}_{a n},\left(\left(\iota_{*} \mathscr{M}\right)_{a n}\right)\right) \mathbb{D R}_{\bar{X}_{a n}} \approx$ $H^{k}\left(\bar{X}, \mathrm{DR}_{\bar{X}}\left(\iota_{*} \mathscr{M}\right)\right)$ follows from a result of Serre [Se]. Finally we have $H^{k}\left(\bar{X}, \mathrm{DR}_{\bar{X}}\left(\iota_{*} \mathscr{M}\right)\right) \simeq H^{k}\left(X, \mathrm{DR}_{X}(\mathscr{M})\right)$.

\$3.4. Let us investigate the structure of $\pi \mathscr{M}$ in the following special case:

Let $X$ be an algebraic manifold $\mathbb{C}^{n}$, and let $X^{*}=\mathbb{C}^{* n}=(\mathbb{C}-\{0\})^{n}$ and $Y=X \backslash X^{*}=\bigcup_{j=1}^{n} Y_{j}$, where $Y_{j}=\left\{x \in \mathbb{C}^{n} ; x_{j}=0\right\}$. Let $H$ be a local system on $X^{*}$ with the unipotent monodromy around $Y_{j}$. Then $\mathscr{M}_{a n}=\mathscr{D}_{X_{a n}^{*}} \otimes H$

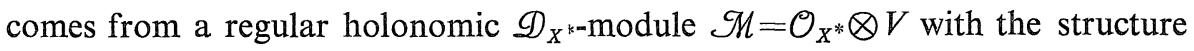
of $\mathscr{D}_{X^{*}-\text { module by }}$

$$
\partial_{j} e=\frac{1}{x_{j}} N_{j} e \quad(j=1, \cdots, n) .
$$

Here $V$ is a finite dimensional $\mathbb{C}$-vector space and $\left\{N_{j}\right\}$ is a set of mutually commuting nilpotent endomorphisms of $V$. Then we have the following

Lemma 3.4.1. $\pi \mathscr{M}$ is an $\mathcal{O}_{X}$-submodule of $j_{*} \mathscr{M}$ given by

$$
\sum_{J \subset I} \mathcal{O}\left[\frac{1}{x_{J}}\right] \operatorname{Im} N_{J}
$$


Here $I=\{1, \cdots, n\}$ and $x_{\boldsymbol{s}}=\prod_{j \in J} x_{j}, N_{J}=\prod_{j \in J} N_{j}$. Of course, we understand $x_{\phi}=1, N_{\phi}=1$.

Proof. It is easy to see that $\mathscr{I}=\sum \mathcal{O}\left[\frac{1}{x_{J}}\right] \operatorname{Im} N_{J}$ is the $\mathscr{D}_{X}$-submodule of $j_{*} \mathscr{M}$ generated by $\mathcal{O V}$. Suppose that there is a surjective morphism $\varphi: \mathscr{I} \rightarrow \mathcal{L}$ to a coherent $\mathscr{D}_{X}$-module supported in $Y$. For any $v \in V$, we have, for a sufficiently large $m$,

$$
\left(x_{j} \partial_{j}\right)^{m}(1 \otimes v)=0 \quad \text { for } j=1, \cdots, n .
$$

On the other hand, $u \underset{\text { def }}{=} \varphi(1 \otimes v)$ satisfies $\left(x_{1} \cdots x_{n}\right)^{m} u=0$, and hence

$$
0=\left(\partial_{1} \cdots \partial_{n}\right)^{m}\left(x_{1} \cdots x_{n}\right)^{m} u=\prod_{\substack{1 \leqq j \leqq n \\ 1 \leqq \nu \leqq m}}\left(x_{j} \partial_{j}+\nu\right) u
$$

Together with $\left(x_{j} \partial_{j}\right)^{m} u=0(j=1, \cdots, n)$, we then obtain $u=0$. Hence $\varphi=0$, that is, $\mathcal{L}=0$. Since $\mathscr{\Omega}$ has no non-zero coherent $\mathscr{D}_{X}$ - submodule supported in $Y$, this completes the proof of the lemma.

Let ${ }^{\pi} H$ denote the minimal extension of $H$ in the category of perverse complexes; that is, ${ }^{\pi} H$ is a perverse complex on $X$ such that its restriction to $X^{*}$ coincides with $H$ and that there exists (in the category of perverse complexes) neither non-zero quotient nor non-zero subobject of ${ }^{\pi} H$ having its support in $Y$. Then we have

$$
{ }^{\pi} H \cong \mathrm{DR}_{X_{a n}}\left({ }^{\pi} \mathscr{M}_{a n}\right) .
$$

Further we have the following quasi-isomorphisms:

$$
\begin{aligned}
& \left({ }^{\pi} H\right)_{0} \leftleftarrows \mathbb{R} \Gamma\left(X_{a n} ;{ }^{\pi} H\right) \\
& \text { १| } \mid 1 \\
& \mathrm{DR}_{X_{a n}}\left({ }^{\pi} \mathscr{M}_{a n}\right)_{0} \leftleftarrows \mathbb{R} \Gamma\left(X_{a n} ; \mathbb{D R}_{X_{a n}}\left(\pi \mathscr{M}_{a n}\right)\right) \\
& ? \uparrow \\
& \mathbb{R} \Gamma\left(X ; \mathbb{D R}_{X}\left({ }^{\pi} \mathscr{M}\right)\right)=\Gamma\left(X ; \mathbb{D R}_{X}\left({ }^{\pi} \mathscr{M}\right)\right) \text {. }
\end{aligned}
$$

Here $\left({ }^{\pi} H\right)_{0}$ etc. denote the germ at 0 of ${ }^{\pi} H$ etc.

Let $\Pi\left(N_{1}, \cdots, N_{n}\right)$ be the subcomplex of $\Gamma\left(X ; D \mathbb{R}_{X}(\pi \mathscr{H})\right)$ given by

$$
\Pi^{k}\left(N_{1}, \cdots, N_{n}\right)=\bigoplus_{\substack{J \in I \\|J|=k}}\left(\operatorname{Im} N_{J}\right) \underset{j \in J}{\mathbb{I}} \frac{d x_{j}}{x_{j}} \subset \Gamma\left(X ; \Omega_{X}^{k} \otimes^{\pi} \mathscr{M}\right)
$$

Remark 3.4.2. Let $W$ be the $n$-dimensional vector space with the basis $\left\{e_{1}, \cdots, e_{n}\right\}$. Then the complex $K\left(N_{1}, \cdots, N_{n}\right)$ given by 


$$
K\left(N_{1}, \cdots, N_{n}\right)^{k}=V \otimes \stackrel{k}{\wedge} W
$$

with the exterior product with $\sum N_{i} e_{i}$ as the differential is called the Koszul complex. The complex $\Pi\left(N_{1}, \cdots, N_{n}\right)$ can be identified with the subcomplex of $K\left(N_{1}, \cdots, N_{n}\right)$ given by $\sum_{J \subset I}\left(\operatorname{Im} N_{J}\right) \otimes \bigwedge_{j \in J} e_{j}$, where $e_{j}=d x_{j} / x_{j}$. Hence we call $\Pi\left(N_{1}, \cdots, N_{n}\right)$ the partial $\mathbb{K}$ oszul complex.

Proposition 3.43.3. $\Pi\left(N_{1}, \cdots, N_{n}\right)$ is quasi-isomorphic to $\Gamma\left(X ; \mathbb{D R}_{X}(\pi \mathscr{H})\right)$.

Corollary 3.4.48 $\Pi\left(N_{1}, \cdots, N_{n}\right)$ is quasi-isomorphic to $\left({ }^{\pi} H\right)_{0}$.

Proof. The vector field $x_{j} \partial_{j}$ operates on $\mathbb{T}\left(X ; \mathbb{D R}_{X}\left({ }^{\pi} \mathscr{M}\right)\right)$ by the $\mathbb{L i e}$ derivative $L_{x_{j} \partial_{j}}$ of $x_{j} \partial_{j}$. For $m=\left(m_{1}, \cdots, m_{n}\right) \in \mathbb{Z}^{n}$, set

$$
\begin{gathered}
\left(\Gamma\left(X ; \mathrm{DR}_{X}(\pi \mathscr{M})\right)\right)_{m}=\left\{u \in \Gamma\left(X ; \mathbb{D R}_{X}(\pi \mathscr{M})\right) ;\left(L_{x_{j} \partial_{j}}-m_{j}\right)^{c} u=0\right. \\
\text { for } c \gg 0, j=1, \cdots, n\} .
\end{gathered}
$$

Then the complex $\Gamma\left(X ; \mathrm{DR}_{X}\left({ }^{\pi} \mathscr{M}\right)\right)$ decomposes as follows:

$$
\Gamma\left(X ; \mathbb{D R}_{X}(\pi \mathscr{M})\right)=\bigoplus_{m \in Z^{n}} \Gamma\left(X ; \mathbb{D R}_{X}(\pi \mathscr{M})\right)_{m} \text {. }
$$

Since we have $\Pi\left(N_{1}, \cdots, N_{n}\right)=\Gamma\left(X ; \mathbb{D R}_{X}(\pi \mathscr{M})\right)_{0}$, it is enough to show that $\Gamma\left(X ; \mathrm{DR}_{X}(\pi \mathscr{M})\right)_{m}$ is homotopically equivalent to 0 if $m \neq 0$. Since

$$
L_{x_{j} \partial_{j}}=d i_{x_{j} \partial_{j}}+i_{x_{j} \partial_{j}} d
$$

holds for the interior product $i_{x_{j} \partial_{j}}$ of the vector field $x_{j} \partial_{j}, L_{x j} \partial_{j}$ is homotopically equivalent to 0 . On the other hand, $\left(L_{x_{j} \partial_{j}}-m_{j}\right)^{c}=0$ holds for $c \gg 0$ on $\Gamma\left(X ; \mathbb{D}_{X}(\pi \mathscr{M})\right)_{m}$ by the definition. Hence

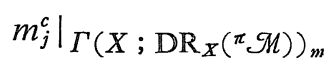

is homotopically equivalent to 0 . This proves the required result: If $m_{j} \neq 0$ for some $j$, then $\Gamma\left(X ; \mathbb{D}_{X}(\mathscr{M})\right)_{m}$ is homotopically equivalent to 0 .

The corollary is an immediate consequence of the proposition and (3.4.1).

Q.E.D.

\section{§4. Purnity Theorem}

$\S \mathbb{H}_{0}$. Let $\left\{S ; H, F, \bar{F} ; N_{1}, \cdots, N_{l}\right\}$ be a nilpotent orbit of weight $w$. Using the mixed Hodge structure of $H$ given by the weight filtration $W\left(N_{1}, \cdots, N_{l}\right)$, we endow the partial Koszul complex $\Pi\left(N_{1}, \cdots, N_{l}\right)$ (cf. §3.4) with mixed Hodge structure as follows: 


$$
\begin{aligned}
& W_{k}\left(\operatorname{Im} N_{j_{1}} \cdots N_{j_{q}}\right)=N_{j_{1}} \cdots N_{j_{q}}\left(W_{k-w}\left(N_{1} \cdots, N_{l}\right)\right), \\
& F^{p}\left(\operatorname{Im} N_{j_{1}} \cdots N_{j_{q}}\right)=N_{j_{1}} \cdots N_{j_{q}} F^{p}(H), \\
& \bar{F}^{p}\left(\operatorname{Im} N_{j_{1}} \cdots N_{j_{q}}\right)=N_{j_{1}} \cdots N_{j_{q}} \bar{F}^{p}(H) .
\end{aligned}
$$

The purpose of this section is to prove the following theorem concerning the weight of $H^{k}\left(\Pi\left(N_{1}, \cdots, N_{l}\right)\right)$.

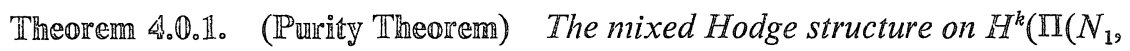
$\left.\cdots, N_{l}\right)$ ) given above is of weight equal io or less than $w^{\prime}+k$, that is, $\operatorname{Gr}_{j}^{W}\left(H^{k}\left(\Pi\left(N_{1}, \cdots, N_{l}\right)\right)=0\right.$ for $j>w+k$.

In order to state the dual statement of the purity theorem, we introduce the following complex $\Pi *\left(N_{1}, \cdots, N_{l}\right)$ :

$$
\Pi^{*}\left(N_{1}, \cdots, N_{l}\right)_{k}=\bigoplus_{|J|=k}\left(\operatorname{Im} N_{J}\right) e_{J} \subset H \underset{Z}{\otimes} \wedge \mathbb{Z}^{l}
$$

where $e_{1}, \cdots, e_{l}$ is the base of $\mathbb{Z}^{l}$ and $e_{J}=\bigwedge_{j \in J} e_{j}$. The differential

$$
\Pi *\left(N_{1}, \cdots, N_{l}\right)_{k} \rightarrow \Pi *\left(N_{1}, \cdots, N_{l}\right)_{k-1}
$$

is given by the inner product with $\sum_{i=1}^{l} e_{i}$. We endow with $\Pi^{*}\left(N_{1}, \cdots, N_{l}\right)$ the mixed Hodge structure by

$$
\left.W_{k} \Pi *\left(N_{1}, \cdots, N_{l}\right)\right)=\oplus\left(\left(\operatorname{Im} N_{J}\right) \cap W_{k-w}\left(N_{1}, \cdots, N_{l}\right)\right) e_{J}
$$

and

$$
\begin{aligned}
& F^{p}\left(\Pi^{*}\left(N_{1}, \cdots, N_{l}\right)\right)=\oplus\left(\left(\operatorname{Im} N_{J}\right) \cap F^{b}(H)\right) e_{J} \\
& \bar{F}^{p}\left(\Pi^{*}\left(N_{1}, \cdots, N_{l}\right)\right)=\oplus\left(\left(\operatorname{Im} N_{J}\right) \cap \bar{F}^{p}(H)\right) e_{J}
\end{aligned}
$$

Then we have, as the dual statement of the purity theorem,

Theorem 4.0.2. The weight of $H_{k}\left(\Pi *\left(N_{1}, \cdots, N_{l}\right)\right)$ is equal to or greater than $w-k$.

$\S 4_{0} \mathbb{1}_{\text {. }}$ Before starting the proof of Theorem 4.0.1, we note the following:

(4.1.1) $\quad H^{q}\left(\Pi\left(N_{1}, \cdots, N_{l}\right)\right)=0, \quad H_{q}\left(\Pi *\left(N_{1}, \cdots, N_{l}\right)\right)=0 \quad$ for $q \geqq l$,

(4.1.2) $\quad H^{0}\left(\Pi\left(N_{1}, \cdots, N_{l}\right)\right)=\operatorname{Ker} N_{1} \cap \cdots \cap \operatorname{Ker} N_{l}$,

(4.1.3) $H^{l-1}\left(\Pi\left(N_{1}, \cdots, N_{l}\right)\right)=\frac{\operatorname{Ker} N_{1} \cdots N_{l}}{\sum_{j=1}^{1} \operatorname{Ker} N_{1} \cdots N_{j-1} N_{j+1} \cdots N_{l}}$,

$$
H_{l-1}\left(\Pi^{*}\left(N_{1}, \cdots, N_{l}\right)\right)=\frac{\bigcap_{j=1}^{l} \operatorname{Im} N_{1} \cdots N_{j-1} N_{j+1} \cdots N_{l}}{\operatorname{Im} N_{1} \cdots N_{l}} .
$$


Therefore the theorem is trivial for $q=0$ or $l=1$.

On the other hand, the purity theorem in $q=l-1$ implies

Corollary 4.1.1. $\quad \operatorname{Ker} N_{1} \cdots N_{l} \subset \operatorname{Ker} N_{1} \cdots N_{l} \cap W_{l-1}\left(N_{1}, \cdots, N_{l}\right)+$ $\sum_{j=1}^{l} \operatorname{Ker} N_{1} \cdots N_{j-1} N_{j+1} \cdots N_{l}$ and $\left(\bigcap_{j=1}^{l} \operatorname{Im} N_{1} \cdots N_{j-1} N_{j+1} \cdots N_{l}\right) \cap W_{-l}\left(N_{1}, \cdots\right.$, $\left.N_{l}\right) \subset \operatorname{Im} N_{1} \cdots N_{l}$.

For $l=2$, this can be restated as

$$
\operatorname{Im} N_{1} \cap \operatorname{Ker} N_{2} \subset W_{-1}\left(N_{1}, N_{2}\right)+N_{1}\left(\operatorname{Ker} N_{2}\right) .
$$

$\S \$ 2$ Let us first consider the case where the following condition is satisfied:

$$
W\left(N_{l}\right)=W\left(N_{1}, N_{2}, \cdots, N_{l}\right)
$$

This is equivalent to the existence of an open convex cone $C$ in $\sum \mathbb{R} N_{j}$ such that $C \supset C\left(N_{1}, \cdots, N_{l}\right), C \ni N_{l}$ and that $\{F, \bar{F} ; C\}$ forms a nilpotent orbit (in the sense of Definition 1.2.3).

We start our reasoning by noting that the following exact sequence (4.2.2) is obtained by regarding the diagram (4.2.3) below as a double complex that defines $\Pi\left(N_{1}, \cdots, N_{l}\right)$. Here and in what follows, $H_{1}$ denotes $\operatorname{Im} N_{1}$, as usual.

$$
\begin{gathered}
0 \rightarrow \Pi\left(\left.N_{2}\right|_{H_{1}}, \cdots,\left.N_{l}\right|_{H_{1}}\right)[-1] \rightarrow \Pi\left(N_{1}, \cdots, N_{l}\right) \rightarrow \Pi\left(N_{2}, \cdots, N_{l}\right) \rightarrow 0 . \\
\qquad \longrightarrow \bigoplus_{j \geqq 2} \operatorname{Im} N_{j} \longrightarrow \bigoplus_{j, k \geqq 2}^{\prime} \operatorname{Im} N_{j} N_{k} \rightarrow \cdots \\
\quad \operatorname{Im} \stackrel{N}{N}_{1} \rightarrow \bigoplus_{j \geqq 2} \operatorname{Im} N_{1} N_{j} \rightarrow \bigoplus_{j, k \geqq 2}^{\prime} \operatorname{Im} N_{1} N_{j} N_{k} \rightarrow \cdots .
\end{gathered}
$$

Furthermore the assumption (4.2.1) guarantees that the exact sequence (4.2.2) lies in the category of mixed Hodge structures. Note also that the vanishing cycle theorem implies

$$
\begin{aligned}
& \left\{\left.N_{1}\right|_{H_{1}},\left.N_{2}\right|_{H_{1}}, \cdots,\left.N_{l}\right|_{H_{1}}\right\} \text { forms a nilpotent orbit of weight } w+1 \text { with } \\
& W\left(\left.N_{2}\right|_{H_{1}}, \cdots,\left.N_{l}\right|_{H_{1}}\right)=W\left(\left.N_{1}\right|_{H_{1}}, \cdots,\left.N_{l}\right|_{H_{1}}\right)=W\left(\left.N_{l}\right|_{H_{1}}\right) .
\end{aligned}
$$

Let us now prove the theorem by the induction on $l$. We obtain the following exact sequence (4.2.5) from (4.2.2):

$$
H^{k-1}\left(\Pi\left(\left.N_{2}\right|_{H_{1}}, \cdots,\left.N_{l}\right|_{H_{1}}\right)\right) \rightarrow H^{k}\left(\Pi\left(N_{1}, \cdots, N_{l}\right)\right) \rightarrow H^{k}\left(\Pi\left(N_{2}, \cdots, N_{l}\right)\right) .
$$

By the induction hypothesis, the left cohomology group in (4.2.5) has the weight $\leqq(w+1)+(k-1)=w+k$ and the right one has the weight $\leqq w+k$. Therefore the middle one has also the weight $\leqq w+k$. Thus the induction proceeds, and the proof is completed if the condition (4.2.1) is satisfied. 
Next let us prove

$$
\begin{aligned}
& \mathbb{H}^{k}\left(\mathbb{I}\left(N_{1}, \cdots, N_{l}\right)\right) \text { is a quotient of } \\
& \mathbb{H}^{k}\left(\mathbb{I}\left(N_{1}+N_{2}, N_{1}+N_{2}+N_{3}, \cdots, N_{1}+\cdots+N_{l}, N_{\text {I }} \cdots, N_{l}\right)\right) \text { for every } k
\end{aligned}
$$

As it is clear that $\mathbb{H}^{k}\left(\mathbb{I}\left(N_{1}+N_{2}, N_{1}+N_{2}+N_{3}, \cdots, N_{1}+\cdots+N_{l}, N_{1}, \cdots, N_{l}\right)\right)$ satisfies the condition (4.2.1), the verification of (4.2.6) finishes the proof.

To prove (4.2.6), let us first prepare some sublemmas.

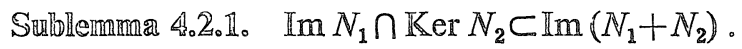

Proof. We prove this by the induction on the dimension of $\mathbb{H}$. Let $N_{0}$ denote $N_{1}+N_{2}$, and denote $\operatorname{Im} N_{0}$ by $H_{0}$. Then the hypothesis of the induction implies

$$
N_{1}\left(H_{0}\right) \cap \mathbb{K e r} N_{2} \subset N_{0}\left(H_{0}\right) \text { 。 }
$$

Hence $N_{0}\left(\operatorname{Im} N_{1} \cap \mathbb{K e r} N_{2}\right) \subset \operatorname{Im} N_{0}^{2}$, or $\operatorname{Im} N_{1} \cap \operatorname{Ker} N_{2} \subset \operatorname{Im} N_{0}+\operatorname{Ker} N_{0}$. Set $W=W\left(N_{0}\right)$. Then

$$
\operatorname{Gr}_{k}^{W}\left(\operatorname{Im} N_{0}+\mathbb{K} \operatorname{er} N_{0}\right)=\operatorname{Grr}_{k}^{W}\left(\operatorname{Im} N_{0}\right)
$$

holds for $k>0$. Hence it is sufficient to show that

$$
\operatorname{Im} N_{1} \cap \operatorname{Ker} N_{2} \cap W_{0} \subset \operatorname{Im} N_{0} \text {. }
$$

Let $x \in W_{2}$ and $N_{1} x \in \mathbb{K e r} N_{2}$. We shall show $N_{1} x \in \operatorname{Im} N_{0}$. We have

$$
N_{1} x=N_{0} y+z \text { with } y \in W_{2} \text { and } z \in \mathbb{K} \operatorname{Rer} N_{0} \text {. }
$$

Setting $y^{\prime}=x-y, z^{\prime}=-z$, we have

$$
N_{2} x=N_{0} y^{\prime}+z^{\prime} \text { 。 }
$$

Since $N_{1} N_{2} x=0$, we have

$$
N_{2} N_{0} y+N_{2} z=0
$$

which implies $N_{0}^{2} N_{2} y=0$. Hence, by setting $W^{\prime}=W\left(\left.N_{0}\right|_{\operatorname{Im} N_{2}}\right)$, we find

$$
N_{2} y \in W_{1}^{\prime} \text {. }
$$

Moreover $N_{2} y \bmod W_{0}^{\prime}$ belongs to $\mathbb{P}_{1}\left(\left.N_{0}\right|_{\operatorname{Im} N_{2}}\right)$, the primitive part with respect to $\left.N_{0}\right|_{\operatorname{Im~} N_{2}}$. Hence we obtain

$$
S_{\text {Im } N_{2}}\left(\mathbb{C N}_{2} y_{9} N_{0} N_{2} y\right) \geqq 0,
$$

that is,

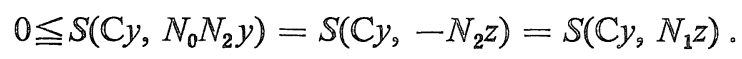


Using (4.2.7) we can also verify

$$
0 \leqq S\left(\mathbb{C} y^{\prime},-N_{1} z^{\prime}\right)=S\left(\mathbb{C} y^{\prime}, N_{1} z\right)
$$

Summing up (4.2.8) and (4.2.9), we find

$$
\begin{aligned}
& 0 \leqq S\left(\mathbb{C} x, N_{1} z\right) \\
& =-S\left(C N_{1} x, z\right) \\
& =-S\left(\mathbb{C}\left(N_{0} y+z\right), z\right)
\end{aligned}
$$

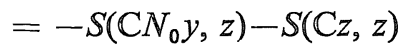

$$
\begin{aligned}
& =\mathbb{S}\left(\mathbb{C} y, N_{0} z\right)-S(\mathbb{C} z, z) \\
& =-S(C z, z) \text {. }
\end{aligned}
$$

Since $z$ is in $\mathbb{P}_{0}\left(N_{0}\right)$. This means $z \in W_{-1} \subset \operatorname{Im} N_{0}$. Therefore $N_{1} x=N_{0} y+z$ also belongs to $\operatorname{Im} N_{0}$.

Q.E.D.

Sublemma 4.2.2. Let $N_{1}$ and $N_{2}$ be mutually commuting endomorphisms of a module $M$. Set $N_{0}=N_{1}+N_{2}$ and suppose

$$
\operatorname{Ker} N_{1} \cap \operatorname{Im} N_{2} \subset \operatorname{Im} N_{0} \text {. }
$$

\section{Then}

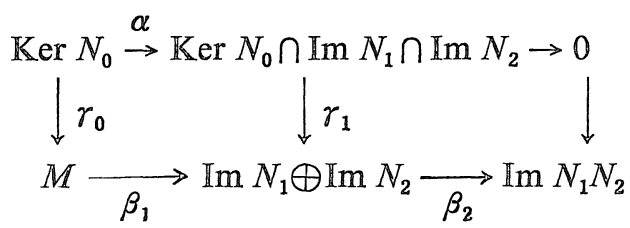

is a morphism of complexes and a quasi-isomorphism. Here

$$
\begin{aligned}
& \alpha(x)=N_{1} x=-N_{2} x, \quad \beta_{1}(x)=\left(N_{1} x, N_{2} x\right), \quad \beta_{2}(x, y)=N_{2} x-N_{1} y, \\
& \gamma_{0}(x)=x, \quad \gamma_{1}(x)=(x,-x) .
\end{aligned}
$$

Proof. Since $\beta_{1} \circ \gamma_{0}=\gamma_{1} \circ \alpha$ and $\beta_{2} \circ \gamma_{1}=0$ are easily verified, this is a morphism of complexes. Since $\operatorname{Ker} \alpha=\operatorname{Ker} N_{1} \cap \operatorname{Ker} N_{2}=\operatorname{Ker} \beta_{1}$, and $\beta_{2}$ is surjective, it is enough to show that $\operatorname{Coker} \alpha \rightarrow \operatorname{Ker} \beta_{2} / \operatorname{Im} \beta_{1}$ is bijective.

(4.2.13) Injectivity: Assume that $x \in \mathbb{K} \operatorname{er} N_{0} \cap \operatorname{Im} N_{1} \cap \operatorname{Im} N_{0}$ satisfies $\gamma_{1}(x)=$ $\beta_{1}(y)$ for $y \in M$. Then $x=N_{1} y=-N_{2} y$. Hence $y \in \mathbb{K e r} N_{0}$ and $x=\alpha(y)$.

(4.2.14) Surjectivity: Assume $\left(x_{1}, x_{2}\right) \in \operatorname{Im} N_{1} \oplus \operatorname{Im} N_{2}$ satisfies $\beta_{2}\left(x_{1}, x_{2}\right)=$ $N_{2} x_{1}-N_{1} x_{2}=0$. Set $x_{i}=N_{i} y_{i}$. Then $N_{1} N_{2}\left(y_{1}-y_{2}\right)=0$, and hence the 
condition ( 4.2 .11$)$ entails

$$
N_{2}\left(y_{2}-y_{1}\right) \in \mathbb{K} \operatorname{er} N_{1} \cap \operatorname{Im} N_{2} \subset \operatorname{Im} N_{0} \text { 。 }
$$

Therefore there is $z \in \mathbb{N}$ such that

$$
N_{2}\left(y_{2}-y_{1}\right)=N_{0} z
$$

Setting $u=N_{2}\left(y_{2}-y_{1}-z\right)$, we find $u=N_{1} z$, and hence $u \in \operatorname{Im} N_{1} \cap \operatorname{Im} N_{2}$. Furo thermore $N_{0} u=N_{1} N_{0} z=N_{1} N_{2}\left(y_{2}-y_{1}\right)=0$. Hence we obtain

$$
\left(x_{1}, x_{2}\right)=\beta_{1}\left(z+y_{1}\right)-\gamma_{2}(u)
$$

Now let us prove (4.2.6). First we consider the case where $l=2$ 。 Then we have the following commutative diagram, where $N_{0}=N_{1}+N_{2}$ :

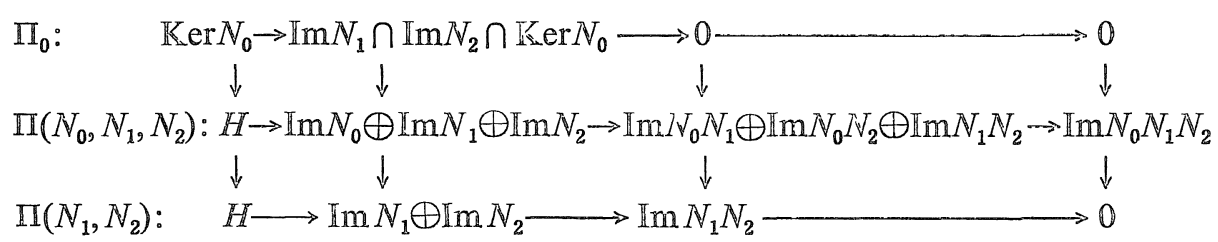

It follows from Sublemma 4.2.2 that the complex $\mathbb{R}_{0}$ given in the top row of (4.2.15) is quasi-isomorphic to the partial $\mathbb{K}$ oszul complex $\mathbb{I}\left(N_{1}, N_{2}\right)$. Hence $H^{k}\left(\Pi\left(N_{1}, N_{2}\right)\right)$ is a direct summand of $H^{k}\left(\mathbb{I}\left(N_{0}, N_{1}, N_{2}\right)\right)$ for every $k$.

In the general situation, let $\mathbb{I}^{\prime}$ denote $\mathbb{I}\left(N_{3}, \cdots, N_{l}\right)$. Then, in parallel with (4.2.15), we find the following commutative diagram:

$$
\begin{gathered}
\left\{\mathbb{\Pi}^{\prime} \cap \mathbb{K e r} N_{0} \rightarrow \mathbb{N}_{1}\left(\mathbb{M}^{\prime}\right) \cap N_{2}\left(\mathbb{M}^{\prime}\right) \cap \operatorname{Ker} N_{0}\right\} \stackrel{v}{\rightarrow} \mathbb{\Pi}\left(N_{1}, N_{2}, N_{3}, \cdots, N_{l}\right) \\
\downarrow \\
\mathbb{\Pi}\left(N_{0}, N_{1}, N_{2}, N_{3}, \cdots, N_{l}\right)
\end{gathered}
$$

where $N_{0}=N_{1}+N_{2}$ and $\sigma$ is a quasi-isomorphism. Hence $\mathbb{H}^{k}\left(\Pi\left(N_{I}, N_{2}, \ldots, N_{l}\right)\right)$ is a direct summand of $\mathbb{H}^{k}\left(\mathbb{I}\left(N_{1}+N_{2}, N_{1}, N_{2}, N_{3}, \cdots, N_{b}\right)\right)$. Repeating this procedure we finally find (4.2.6). At long lastis, this completes the proof of the purity theorem.

\section{§5. $\mathbb{I}^{2}$-Cohomology Groups amd Imatersection Colnomology Grounps}

§5.1. Let $X$ be a compact complex manifold, $Y$ a normally crossing hyper: 
surface. We set $X^{*}=X \backslash Y$. Let $H$ be a variation of polarized Hodge structure of weight $w$ on $X^{*}$. We assume

(5.1.1) $H_{\boldsymbol{C}}$ is quasi-unipotent, i.e., the local monodromy of $H_{\boldsymbol{C}}$ around any irreducible component of $Y$ is quasi-unipotent.

Let $\mathrm{C}(x)$ denote the Weil operator of the Hodge structure $\left(H_{C, x}, F(x), \bar{F}(x)\right)$ and define the inner product $\langle f \mid g\rangle_{x}\left(f, g \in H_{C, x}\right)$ by $S_{x}(\mathrm{C}(x) f, \bar{g})$. Then the $C^{\infty}$-vector bundle on $X^{*}$ associated with the local system $H_{C}$ is equipped with the Hermitian metric defined by this inner product.

\$5.2. To consider $L^{2}$-cohomology groups, let us equip $X^{*}$ with a Riemannian metric $g$ which behaves on a neighborhood of $Y$ as follows:

Let $y_{0}$ be a point of $Y$ and choose a local coordinate system $\left(z_{1}, \cdots, z_{n}\right)$ such that $Y$ is defined by $z_{1} \bullet z_{l}=0$ in a neighborhood of $y_{0}$. Then

$$
g \sim \sum_{j \leqq l} \frac{\sqrt{-1} d z_{j} d \bar{z}_{j}}{\left(\left|z_{j}\right| \log \left|z_{j}\right|\right)^{2}}+\sum_{j>l} \sqrt{-1} d z_{j} d \bar{z}_{j} .
$$

Here, and in what follows the symbol $\sim$ means that each of the two metrics is bounded by a constant mulitiple of the other on a neighborhood of the point in question. One can easily show (ef. [Z], §3) that such a metric exists. Furthermore, as shown in $[\mathbb{Z}]$, Proposition 3.4, it enjoys the following properties.

(5.2.2) It is complete and the volume of $X^{*}$ is finite.

(5.2.3) The supremum of any $C^{\infty}$-form on $X$ is finite with respect to the metric.

§5.3. As we have thus introduced a Riemannian metric on $X^{*}$ and a Hermitian metric on $H_{C}$, we are now ready to define the $L^{2}$-cohomology groups.

In order to give their precise definitions, let us prepare some notations.

Let $\mathscr{D} k_{x}^{p}$ denote the sheaf of distribution-valued $p$-forms, and let $\mathscr{D} k_{X *}^{p}(H)$

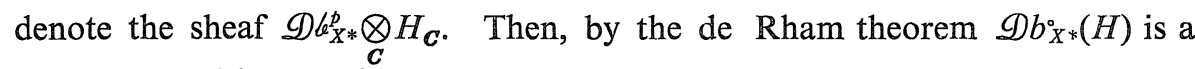
complex quasi-isomorphic to $H_{C}$.

Now we introduce the sheaf $\mathcal{L}^{p}(H)$ by the following

Defimition 5.3.1. For any open subset $U$ of $X, \Gamma\left(U ; \mathcal{L}^{p}(H)\right)$ is, by definition, the totality of $u$ in $\Gamma\left(U \backslash Y, \mathscr{D} k^{p}(H)\right)$ such that, for any compact set $K$ of $U$, both $u$ and its exterior derivative $d u$ are square-integrable on $K \cap X^{*}$ with respect to the Riemannian metric $g$ on $X^{*}$ and the Hermitian metric on the vector bundle $H$. 
The sheaf $\mathcal{L}^{p}(H)$ thus defined does not depend on the choice of the Riemannian metric $g$ on $X^{*}$, provided that $g$ satisfies the condition (5.2.1). It is also clear that $\mathcal{L}^{\circ}(H)$ forms a subcomplex of $j_{*} \mathscr{D} b_{X^{*}}(H)$, where $j$ denotes the inclusion map $X^{*} \hookrightarrow X$.

Furthermore we have the following

Lemma 5.3.2. $\mathcal{L}^{p}(H)$ is a soft sheaf.

In fact, for $u \in \Gamma\left(U ; \mathcal{L}^{p}(H)\right)$ and $\varphi \in C_{0}^{\infty}(U)$, both $\varphi u$ and $d(\varphi u)=\varphi d u+d \varphi u$ are square-integrable because $\varphi$ and $d \varphi$ have finite norms. Therefore $\mathcal{L}^{p}(H)$ is a fine sheaf and hence soft. (See [G].)

If we denote by $L_{(2)}^{p}\left(X^{*} ; H_{C}\right)$ the set of $H_{C}$-valued $L^{2}$-p-forms on $X^{*}$, then we find

$$
\Gamma\left(X ; \mathcal{L}^{p}(H)\right)=\left\{u \in L_{(2)}^{p}\left(X^{*} ; H_{C}\right) ; d u \in L_{(2)}^{p+1}\left(X^{*} ; H_{C}\right)\right\}
$$

Hence Lemma 5.3.2 entails

$$
\begin{aligned}
H^{p}\left(X ; \mathcal{L}^{\circ}(H)\right) & =\frac{\mathbb{K e r}\left(d: \Gamma\left(X ; \mathcal{L}^{p}(H)\right) \rightarrow \Gamma\left(X ; \mathcal{L}^{p+1}(H)\right)\right)}{\operatorname{Im}\left(d: \Gamma\left(X: \mathcal{L}^{p-1}(H)\right) \rightarrow \Gamma\left(X: \mathcal{L}^{p}(H)\right)\right)} \\
& =\frac{\left\{u \in L_{(2)}^{p}\left(X^{*} ; H_{C}\right) ; d u=0\right\}}{\left\{d u ; u \in L_{(2)}^{p-1}\left(X^{*} ; H_{C}\right) \text { such that } d u \in L_{(2)}^{p}\left(X^{*} ; H_{C}\right)\right\}}
\end{aligned}
$$

We call this the $p$-th $L^{2}$-cohomology group. We sometimes use the abbreviated notation $H_{(2)}^{p}\left(X^{*} ; H\right)$ to denote $H^{p}\left(X ; \mathcal{L}^{\circ}(H)\right)$.

$\$ 5.4$. We can now state the main result of this article:

Theorem 5.4.1. The complex $\mathcal{L}^{\circ}(H)$ is quasi-isomorphic to ${ }^{\pi} H_{C}$, the minimal extension of $H_{C}$ introduced in $\$ 3.4$. In particular, the $L^{2}$-cohomology group $H^{k}\left(X ; \mathcal{L}^{\circ}(H)\right)$ coincides with the intersection cohomology group $H^{k}\left(X ;{ }^{\pi} H_{C}\right)$.

\$5.5. For any point $x \in X$, there exists an open neighborhood $U$ of $x$ and a family of holomorphic functions $\left\{f_{j}\right\}_{j \in I}$ defined on $U$ such that $\left\{d f_{j}(x)\right\}_{j \in I}$ is linearly independent and $Y \cap U=\bigcup_{j \in I}\left\{x \in U ; f_{j}(x)=0\right\}$. For $l \geqq 0$, we define $X_{l}$ so that

$$
X_{l} \cap U=\underset{\substack{J \subset I \\|J|=l}}{\bigcup} \bigcap_{j \in J} f_{j}^{-1}(0)
$$

and we set

$$
X_{l}^{*}=X_{b} \backslash X_{l+1}
$$


Then $X_{l}^{*}$ is an $l$-codimensional submanifold of $X$ and $X=\bigsqcup_{l} X_{l}^{*}$ is a Whitney stratification of $X$.

Now, by a classical theorem, we know

$$
\left.H_{C} \rightarrow \mathcal{L}^{\circ}(H)\right|_{X^{*}} \text { is a quasi-isomorphism. }
$$

Therefore we can use the following characterization of ${ }^{\pi} \mathbb{H}_{\mathbb{C}}$ to prove Theorem 5.4.1.

Lemma $5.5 . H_{0}{ }^{\pi} H_{C}$ is a complex of sheaves on $X$ which satisfies the following conditions:

$$
\left.{ }^{\pi} H_{C}\right|_{X^{*}} \text { is quasi-isomorphic to } H_{C} \text {. }
$$

(5.5.3) If we denote by $j_{l}$ the embedding $X \backslash X_{l} \rightarrow X \backslash X_{l+1}$, then

$$
\left.\mathscr{H}^{\nu}\left({ }^{\pi} H_{\overparen{C}}\right)\right|_{x_{l}^{*}}=0 \quad \text { for } \quad \nu \geqq l \geqq 1
$$

and

$$
\left.\left.\mathscr{H}^{\nu}\left(\mathbb{R}^{\mathbb{R}} j_{l *}\left(\left.{ }^{\pi} \mathbb{H}_{\mathbb{C}}\right|_{X \backslash X_{l}}\right)\right)\right|_{x_{l}^{*}} \approx \mathscr{H}^{\nu}\left({ }^{\pi} H_{\mathbb{C}}\right)\right|_{x_{l}^{*}} \quad \text { for } \quad \nu \leqq l-1 .
$$

See Goresky-MacPherson [GM] for the proof of Lemma 5.5.1.

In view of Lemma 5.5.1, the proof of Theorem 5.4.1 is reduced to verifying the following for $l \geqq 1$.

$$
\begin{aligned}
& \text { For } x \in X_{l}^{*}, \mathscr{H}^{\nu}\left(\mathcal{L}^{\circ}(H)\right)_{x}=0 \text { for } \nu \geqq l \text {. } \\
& \text { For } x \in X_{l}^{*} \text {, we have } \\
& \mathcal{H}^{\nu}\left(\mathcal{L}^{\circ}(H)\right)_{x} \underset{\square}{\underset{\sigma \ni x}{\lim }} H^{\nu}\left(U \backslash X_{l} ; \mathcal{L}^{\circ}(H)\right) \quad \text { for } \quad \nu \leqq l-1,
\end{aligned}
$$

where $U$ ranges over a neighborhood system of $x$.

We shall proceed by the induction on $l$, and we may assume from the beginning

$$
\left.\left.\mathcal{L}^{\circ}(H)\right|_{X \backslash X_{l}} \simeq{ }^{\pi} H_{C}\right|_{X \backslash X_{l}} \text { 。 }
$$

In the rest of this section, we prove (5.5.4) and (5.5.5) under the assumption (5.5.6).

Since the question is local, we consider the problem locally on $X$ so that we may assume $X_{l+1}=\phi$. Thus on a neighborhood of $x$, we may assume

$$
\begin{aligned}
& X=\Delta^{l} \times \Delta^{n-b}, \\
& X^{*}=\Delta^{* l} \times \Delta^{n-l},
\end{aligned}
$$


where $\Delta=\{z ;|z|<1\}$ is the unit disc and $\Delta^{*}$ is the punctured disc $\Delta \backslash\{0\}$ 。

\$5.6. Let us begin our discussion by showing the following

Lemmma 5.6.1. Let $M$ be a Riemannian manifola equipped with a metric $g_{0}$. Let I be an open interval $\{t \in \mathbb{R} ;-1<t<1\}$. Suppose that $\mathbb{I} \times M$ is equipped with a Riemannian metric $g$. Let $H$ be a local system on $M$ and let $p$ be the projection $I \times M \rightarrow M$. Suppose that the $C^{\infty}$-vector bundle associated with $p^{-1} H$ is equipped with a Hermitian metric $\|*\|_{(t, x)}$. Suppose

$$
g \sim d \imath^{2}+g_{0} .
$$

Suppose also that

$$
\|*\|_{(t, x)} \sim\|*\|_{(0, x)}
$$

Then we have

$$
H_{(2)}^{k}(M ; H) \cong H_{(2)}^{k}\left(I \times M ; p^{-1} H\right) .
$$

Proof. We may assume without loss of generality that $g=d t^{2}+g_{0}$ and $\|*\|_{(t, x)}=\|*\|_{(0, x)}$. Since an $L^{2}$-form (resp., closed $L^{2}$-form) on $M$ can be trivially extended as an $L^{2}$-form (resp., closed $L^{2}$-form) on $I \times M$, we can define a map

$$
i: H_{(2)}^{k}(M ; H) \rightarrow H_{(2)}^{k}\left(I \times M ; p^{-1} H\right) .
$$

It is easy to verify that $i$ is injective. Iet us show that $i$ is surjective. Let $\tilde{u}(t, x) \underset{\text { def }}{=} u(t, x)+d t v(t, x)$ be a closed form on $I \times M$, where $u$ and $v$ are free from $d t$. Since $u$ is supposed to be closed, we find

$$
\frac{\partial u}{\partial t}=d_{x} v .
$$

Let us take a $C^{\infty}$-function $\varphi(i)$ with compact support in $I$ such that $\int \varphi(t) d i=1$. Set $\psi(t)=\int_{-\infty}^{t} \varphi(s) d s$. Then $\psi(t)=0$ for $t<-1+\varepsilon$ and 1 for $t>1-\varepsilon$. Now we set

$$
w(t)=\int_{-\infty}^{i} \psi(s) v(s) d s-\int_{t}^{\infty}(1-\psi(s)) v(s) d s .
$$

Then (5.6.4) implies

$$
\begin{aligned}
d_{x} w(t) & =\int_{-\infty}^{t} \psi(s) \frac{\partial u(s)}{\partial s} d s-\int_{t}^{\infty}\left(1-\psi(s) \rho \frac{\partial u(s)}{\partial s} d s\right. \\
& =\psi(t) u(t)-\int_{-\infty}^{t} \varphi(s) u(s) d s+(1-\psi(t)) u(t)
\end{aligned}
$$




$$
\begin{array}{r}
+\int_{t}^{\infty}(-\varphi(s)) u(s) d s \\
=u(t)-\int \varphi(s) u(s) d s
\end{array}
$$

and

$$
\frac{\partial}{\partial t} w(t)=\psi(t) v(t)+(1-\psi(t)) v(t)=v(t)
$$

This implies

$$
\tilde{u}=d w+\int \varphi(s) u(s) d s
$$

Since $\int \varphi(s) u(s) d s$ is square-integrable, $w$ and $d w$ are also square-integrable. Hence $\tilde{u}$ is cohomologous to $\int \varphi(s) u(s) d s$. Thus, the map $i$ is surjective. Q.E.D.

\$.\%. Before going further, we show that it suffices to prove Theorem 5.4.1 in the case where the local monodromies of $H_{C}$ are unipotent.

Let $X^{*}$ be $\Delta^{* l} \times \Delta^{n-l}$, where $\Delta^{*}$ is the punctured disc and $\Delta$ is the open disc. For a variation of polarized Hodge structure $H$ on $X^{*}$ with quasi-unipotent monodromies, let $f: X^{\prime} \rightarrow X$ be the covering $\left(z_{1}, \cdots, z_{n}\right) \mapsto\left(z_{\mathrm{I}}^{m}, \cdots, z_{l}^{m}, z_{l+1}, \cdots, z_{n}\right)$ so that $H^{\prime}=f^{-1}(H)$ on $X^{\prime *}=f^{-1}\left(X^{*}\right)$ has unipotent monodromies. Let $G=(\mathbb{Z} / m \mathbb{Z})^{l}$ be the Galois group of the covering $X^{* *} \rightarrow X^{*}$. Then $\mathbb{R} f_{*}\left({ }^{\pi} H_{\mathbb{C}}^{\prime}\right)$ is also a perverse complex and it coincides with ${ }^{\pi}\left(f_{*} H_{C}^{\prime}\right)$. Hence, ${ }^{\pi} H_{C}$ coincides with $G$-invariants of $\mathbb{R} f_{*}\left({ }^{\pi} H_{C}^{\prime}\right)$. Similarly $G$ acts on $f_{*} \mathcal{L}^{\circ}\left(H^{\prime}\right)$ and $\mathcal{L}^{\circ}(H)$ coincides with $G$-invariants of $f_{*} \mathcal{L}^{\circ}\left(H^{\prime}\right)$. Therefore ${ }^{\pi} H^{\prime} \simeq \mathcal{L}^{\circ}\left(H^{\prime}\right)$ implies ${ }^{\pi} H \simeq \mathcal{L}^{\circ}(H)$. Hence we may assume from the beginning that $H$ is with unipotent monodromies.

\section{\$5.8. Comerete Description of the Metrics}

In order to find the concrete description of the $L^{2}$-cohomology groups in terms of harmonic forms (Lemma 5.10.1), we do some preliminary considerations on the metrics and related operators.

First let us try to find a coordinate system on $X$ so that the metric $g$ and the norm on $H_{C}$ may take suitable forms for our purpose.

Thanks to Lemma 5.6.1, we may suppose $l=n$, i.e.,

$$
X=\Delta^{n}, \quad X^{*}=\Delta^{* n} \quad \text { and } \quad g=\frac{\sqrt{-1}}{2} \sum_{j=1}^{n} \frac{d z_{j} d \bar{z}_{j}}{\left(\left|z_{j}\right| \log \left|z_{j}\right|\right)^{2}} .
$$

Let us take a coordinate system $(t, x, y)=\left(t, x_{1}, \cdots, x_{n}, y_{1}, \cdots, y_{n}\right)$ of $X^{*}$, 
with $\sum y_{j}=1$, by

$$
z_{j}=\exp \left(2 \pi \sqrt{-1} x_{j}-\left(t y_{j}\right)^{-1}\right)
$$

Then $X^{*}$ is isomorphic to

$$
\left\{(i, x, y) \in \mathbb{R} \times\left(\mathbb{R}^{n} \mid \mathbb{Z}^{n}\right) \times \mathbb{R}^{n} ; t, y_{1}, \cdots, y_{n}>0, \sum y_{j}=1\right\} .
$$

Now, using the coordinate system $(t, x, y)$, we find the following

\section{Lemma 5.8.1. The Riemannian metric $g$ is equivalent to}

$$
\frac{d t^{2}}{t^{2}}+\sum_{j=1}^{n} t^{2} y_{j}^{2} d x_{j}^{2}+\sum_{j=1}^{n} \frac{d y_{j}^{2}}{y_{j}^{2}}
$$

on $X^{*}$.

Proof. The metric $g$ takes the following form on $X^{*}$ :

$$
\sum_{j} \frac{d x_{j}^{2}+\left(d\left(t y_{j}\right)^{-1}\right)^{2}}{\left(t y_{j}\right)^{-2}}=t^{2} \sum_{j} y_{j}^{2} d x_{j}^{2}+\sum_{j}\left(\frac{d t}{t}+\frac{d y_{j}}{y_{j}}\right)^{2} \text {. }
$$

On the other hand, it follows from the constraint $\sum_{j} y_{j}=1$ that

$$
\sum_{j} d y_{j}=0
$$

Hence we have

$$
\begin{aligned}
\left|\sum_{j} \frac{d y_{j}}{y_{j}}\right|^{2} & =\left|\sum_{j}\left(1-y_{j}\right) \frac{d y_{j}}{y_{j}}\right|^{2} \\
& \leqq\left(\sum_{j}\left(1-y_{j}\right)^{2}\right)\left(\sum_{j} \frac{d y_{j}^{2}}{y_{j}^{2}}\right) \\
& \leqq(n-1)\left(\sum_{j} \frac{d y_{i}^{2}}{y_{j}^{2}}\right) .
\end{aligned}
$$

This shows

$$
\sum_{j}\left(\frac{d t}{t}+\frac{d y_{j}}{y_{j}}\right)^{2} \sim \frac{d t^{2}}{t^{2}}+\sum \frac{d y_{j}^{2}}{y_{j}^{2}}
$$

Q.E.D.

In view of Lemma 5.8.1 we introduce the following family $X^{*}(t)$ of submanifolds of $X^{*}$.

Definition 5.8.2. Let $X^{*}\left(t_{0}\right)$ denote

$$
\left\{(t, x, y) \in X^{*} ; t=i_{0}\right\}
$$

for $t_{0}>0$.

We equip $X^{*}\left(t_{0}\right)$ with the following Riemannian metric $g\left(t_{0}\right)$ : 


$$
g\left(t_{0}\right)=t_{0}^{2}\left(\sum_{j=1}^{n} y_{j}^{2} d x_{j}^{2}\right)+\sum_{j=1}^{n} y_{j}^{-2} d y_{j}^{2} .
$$

Next let us study the Hermitian metric on the fiber bundle $H$. Let $N_{j}$ denote the logarithm of the monodromy of $H_{\mathbb{C}}$ around $\left\{z \in X ; z_{j}=0\right\}$. Since we may assume without loss of generality that $e^{N_{j}}$ is unipotent $(\S 5.7), N_{j}$ is nilpotent. Then $\left\{\exp \left(\sum_{j} x_{j} N_{j}\right) e ; e \in H_{C}\right\}$ is a constant sheaf on $X^{*}$. Let $V$ denote the sections of this sheaf. Then

$$
\varepsilon \otimes H_{C} \simeq \varepsilon \otimes V
$$

and

$$
d(1 \otimes e)=\sum_{j} d x_{j} \otimes N_{j} e \quad \text { for } \quad e \in V .
$$

Here and in what follows, $\mathcal{E}$ denotes the sheaf of $C^{\infty}$-functions.

Now we know the following

Lemma 5.8.3. ([K], [C- $\mathbb{K}-\mathrm{S}, 1])$ Let $K$ be an endomorphism of $V$ which satisfies the following two conditions:

$$
\begin{gathered}
{\left[K, N_{j}\right]=-2 N_{j}, \quad j=1, \cdots, n .} \\
\left.K\right|_{\mathrm{Gr}_{k}^{W}}=k, \text { where } W=W\left(N_{1}, \cdots, N_{n}\right) .
\end{gathered}
$$

Then we have

$$
|e|_{X}(t, x, y) \sim\left|t^{-K / 2} e\right|_{X^{*}}(1, x, y) \quad \text { for } e \in V .
$$

For $e \in V$, if we write $e=\sum e_{k}$ such that $K e_{k}=k e_{k}$, then

$$
|e|_{X^{*}} \sim \sum_{k}\left|e_{k}\right|_{X^{*}}
$$

Here $|*|_{X^{*}}(t, x, y)$ denotes the Hermitian metric of $H_{C}$ at $(t, x, y)$.

Therefore, replacing the fiber metric of $H$ with an equivalent one we may assume the following:

(5.8.9) $|e|_{X^{*}}(t, x, y)$ does not depend on $x$.

(5.8.10) $K$ is a symmetric operator with respect to the metric $|e|_{X^{*}}(t, x, x)$.

Thus we obtain a concrete description of the $L^{2}$-norm of an $H_{C^{-}}$-valued form. To write it down we introduce the following

Definition 5.8 .4$. For $u$ in $\mathscr{D} k^{k}(H), A u$ is, by definition, $(K+2 p) u$ where $p$ denotes the degree of $u$ with respect to $d x_{1} \cdots d x_{n}$. 
In view of Lemma 5.8.3, the $\mathbb{L}^{2}$-norm $\|u\|_{X^{*}}$ is given by

$$
\left(\int\left|\hat{\imath}^{-A / 2} u\right|_{X,(1)}^{2} d \mu_{X}\right)^{1 / 2}
$$

where $d \mu_{X}$ denotes the volume element of $X^{*}$ determined by the metric (5.8.2). Therefore Lemma 5.8.3 entails

$$
\|u\|_{X^{*}}^{2}=\int i^{n}\left\|t^{-A / 2} u\right\|_{X^{*}(1)}^{2} \frac{d \hat{\varepsilon}}{\hat{t}} 。
$$

Since $|d i / t|=1$, for an element $u(t)+v(t) d t / t$ of $L_{(2)}^{k}\left(X^{*} ; H\right), u(t)$ can be regarded as an $L_{(2)}^{k}\left(X^{*}(1) ; \not H\right)$-valued function in $t$ and $v(t)$ is an $L_{(2)}^{k-1}\left(X^{*}(1)\right.$; $H$ )-valued function in $t$ which satisfy

$$
\begin{aligned}
& \int t^{n}\left\|t^{-A / 2} u\right\|_{X^{*}(1)}^{2} d i / \imath<\infty, \\
& \int t^{n}\left\|t^{-A / 2} v\right\|_{X^{r}(1)}^{2} d t / \imath<\infty .
\end{aligned}
$$

The converse being true, we may regard $L_{(2)}^{k}\left(X^{*} ; \mathbb{H}\right)$ as the set of pairs of such $u$ and $v$ that satisfying (5.8.13) and (5.8.14).

We end this subsection by showing the following Lemma 5.8.5.

Lemman 5.8.5. (i) $[A, d]=2 d_{x}$, where $d_{x}$ denotes the differential of the coefficienis of $V$-valued forms, i.e.,

$$
d_{x}(a \otimes e)=\left(d_{x} a\right) \otimes e .
$$

$$
\left[A, d_{X}^{*}(t)\right]=2 \sum_{j}\left(t^{2} y_{j}^{2}\right)^{-1} i_{\partial / \partial x_{j}} L_{\partial / \partial x_{j}}
$$

Here $d_{X^{*}(t)}^{*}$ denotes the adjoint operator of $d$ in ihe Hilberit space $\mathbb{L}_{(2)}^{k}\left(X^{*}(t)\right.$; $\left.\left.H_{C}\right|_{X^{*}(t)}\right)$, and $L_{\partial / \partial x_{j}}\left(\right.$ resp., $\left.i_{\partial \partial / x_{j}}\right)$ denotes the Lie derivaiive (resp., interior prod$u c t$ ), operating on $V$ trivially.

Proof. Let $\varphi$ denote $a \otimes e$ with $e$ in $\mathbb{I}_{k}$. Suppose that $a$ is a $p$-form in $d x_{1}, \cdots, d x_{n}$. Then it follows from the trivialization of $H_{C}$ that

$$
d \varphi=d_{x} a \otimes e+d_{y} a \otimes e+\sum_{j} d x_{j} a \otimes N_{j} e .
$$

Hence it follows from the definition of $A$ and the properties (5.8.4) of $K$ that

$$
\begin{aligned}
A d \varphi= & (k+2(p+1)) d_{x} a \otimes e+(k+2 p) d_{y} a \otimes e \\
& +\sum_{j}(k-2+2(p+1)) d x_{j} a \otimes N_{j} e \\
= & d A \varphi+2 d_{x} a \otimes e .
\end{aligned}
$$


This proves (i).

To prove (ii) let us first note that the symmetric property of $A$ entails

$$
\left[A, d_{X}^{*(t)}\right]=-\left[A, d_{X^{*}(t)}\right]^{*} .
$$

Hence it follows from (i) that

$$
\left[A, d_{x^{*}(t)}^{*}\right]=-2 d_{X}^{*} .
$$

Let us now calculate $d_{X}^{*} \varphi$ for $\varphi=d x_{J} \rho$. Let $\psi=d x_{I} \sigma$. Then

$$
\begin{aligned}
\left(d_{x}^{*} \varphi, \psi\right)_{X^{*}(t)} & =\left(\varphi, d_{x} \psi\right)_{X^{*}(t)} \\
& =\int\left(\varphi, \sum_{j} d x_{j} d x_{I} \frac{\partial \sigma}{\partial x_{j}}\right)_{X^{*}(t)} \\
& =\Sigma \varepsilon(J, j \wedge I) \int\left(\varphi, \frac{\partial \sigma}{\partial x_{j}}\right) t^{-2|J|} \prod_{j \in J} y_{j}^{-2} t^{n} d \mu_{X^{*}(1)}
\end{aligned}
$$

where

$$
\varepsilon(J, j \wedge I)= \begin{cases}\operatorname{sgn}\left(\begin{array}{c}
J \\
j, I
\end{array}\right) & \text { if } J=\{j\} \cup I \\
0 & \text { otherwise. }\end{cases}
$$

Hence we obtain

$$
\left(d_{x}^{*} \varphi, \psi\right)_{X^{*}(t)}=-\sum_{j} t^{-2} y_{i}^{-2}\left(i_{\partial / \partial x_{i}} d x_{I} \frac{\partial \rho}{\partial x_{i}}, \psi\right)_{X^{*}(t)}
$$

This proves (ii).

§5.9. The function $t: X^{*} \rightarrow \mathbb{R}$ extends to $X \backslash\{0\} \rightarrow \mathbb{R}$. Let us denote by $X(t)$ its fiber. Then $X \backslash\{0\}$ is isomorphic to the product of $X(1)$ and $\{t ; t>0\}$. Moreover, $\left.{ }^{\pi} H\right|_{X \backslash(0)}$ is locally constant along the fiber of $X \backslash\{0\} \cong X(1) \times$ $\{t ; t>0\} \rightarrow X(1)$.

It follows from the induction hypothesis (5.5) that

$$
H^{k}\left(L_{\varepsilon} ; \mathcal{L}^{\circ}(H)\right)=H^{k}\left(L_{\varepsilon} ;{ }^{\pi} H_{C}\right)
$$

holds. Here $L_{\varepsilon}$ is the inverse image of $\{t ;|t-1|<\varepsilon\}$ by $t: X \backslash\{0\} \rightarrow \mathbb{R}$. On the other hand, Lemma 5.6.1 entails

$$
H_{(2)}^{k}\left(X^{*}(1) ; H\right) \cong \underset{\varepsilon}{\lim } H^{k}\left(L_{\varepsilon} ; \mathcal{L}^{\circ}(H)\right) \text {. }
$$

Since $H^{k}\left(L_{\varepsilon} ; \mathcal{L}^{\bullet}(H)\right)$ is stable with respect to $\varepsilon,(5.9 .1)$ and (5.9.2) entail

$$
\begin{aligned}
H_{(2)}^{k}\left(X^{*}(1) ; H\right) & \simeq H^{k}\left(X^{*}(1) ;\left.{ }^{\pi} H_{\boldsymbol{C}}\right|_{X^{*}(1)}\right) \\
& \simeq H^{k}\left(X \backslash\{0\} ;{ }^{\pi} H_{\boldsymbol{C}}\right) .
\end{aligned}
$$

In particular, we observe 


$$
\operatorname{dim}_{C} H_{(2)}^{k}\left(X^{*}(1) ; H\right)<\infty
$$

Note that

(5.9.5) $\quad H_{(2)}^{k}\left(X^{*}(1) ; H\right)$

$\simeq\left\{u \in \Gamma\left(X^{*}(1) ; \mathscr{D} \epsilon^{k}\left(\left.H\right|_{X^{*}(1)}\right)\right) ; u\right.$ is square-integrable and $d u=0$ holds in the distribution sense\}

modulo

$\left\{d v ; v \in \Gamma\left(X^{*}(1) ; \mathscr{D} \epsilon^{k-1}\left(\left.H\right|_{X^{*}(1)}\right)\right)\right.$ and both $v$ and $d v$ are square-integrable $\}$

In order to be precise, let us denote by $T_{k}$ the weak closure of the exterior derio vative $d$ acting on $k$-forms. Then (5.9.4) implies that the range $R\left(\mathbb{T}_{k-1}\right)$ of $T_{k-1}$, i.e., the denominator in the right hand side of (5.9.5), is closed. See Appendix for the terminologies and some basic results in functional analysis which we are using here.

Now, the closedness of $R\left(T_{k-1}\right)$ 's guarantees the validity of the HodgeKodaira decomposition ([Ko], Ch. IV, §1. See also Appendix Theorem A.2.1), that is, the following lemma holds.

Lemma 5.9.1. For $t>0$, the space $L_{(2)}^{k}\left(X^{*}(t) ; H\right)$ of $\left(H^{\prime} x^{*}(1)\right)$-valued $L^{2}-k$-forms on $X^{*}(t)$ admits the orthogonal decomposition

$$
R\left(T_{k-1}\right) \oplus h^{k} \oplus R\left(T_{k}^{*}\right),
$$

where $T_{k-1}$ denotes the weak closure of the exterior derivative, $T_{k}^{*}$ denotes the adjoint operator of $T_{k}$, and $h^{k}$ denotes the space of harmonic $L^{2}-k$-forms.

\section{§5.10. HHarmonic Representatives of $\mathbb{L}^{2}-$ Colnomology Groups}

To complete the proof of Theorem 5.4.1 in a later subsection ( $\$ 5.12)$, we prepare a proposition which guarantees that we can choose a harmonic form to represent a cohomology class in $H^{k}\left(X ; \mathcal{L}^{\circ}(H)\right)$. To be more precise, we will prove the following

Proposition 5.10.1. Let $\omega$ be a closed $L^{2}$-form on $X^{*} \cap\{t<a\}$. Then we can find $h(t)$ and $h^{1}(t)$ which are $L_{(2)}\left(X^{*}(1), H\right)$-valued $L^{2}$-form on $\{i ; i<a\}$ satisfying the following conditions:

(5.10.1) $h(t)$ and $h^{1}(t)$ are harmonic. (Cf. Corollary 5.10.5).

(5.10.2) $\omega-\left(h(t)+h^{1}(t) d t / t\right)$ is the coboundary of a square-integrable form on $X^{*} \cap\{t<a\}$.

(5.10.3) $\partial h(t) / \partial t=0$ 
(5.10.4) $h^{1}(t) d t / t$ is a square-integrable form on $X^{*} \cap\{t<a\}$.

In order to prove this proposition, we prepare several notations and lemmas. Let us first denote by $\|f\|_{X^{*}(t)}$ the $\mathbb{L}^{2}$-norm of a distribution $f$ defined on $X^{*}(t)$ which is determined by the Riemannian metric $g(t)$ and the fiber metric on $\left.H^{\prime}\right|_{X^{*}(t)}$. Then it follows from (5.8.3) and (5.8.6) that $\|f\|_{X^{*}(t)}$ and $\|f\|_{X^{*}\left(t^{\prime}\right)}$ are equivalent for each $t$ and each $t^{\prime}\left(0<t \leqq t^{\prime}\right)$. More precisely, there exists a constant $c$ which satisfies

$$
\|f\|_{X^{*}(t)} \leqq\left(\frac{t^{\prime}}{t}\right)^{c}\|f\|_{X^{*}\left(t^{\prime}\right)}
$$

Furthermore we can verify the following

Lemma 5.10.2. There exists a constant $C$ which does not depend on $t<2$ and which enjoys the following property:

For any $L^{2}$-coboundary form $u$ on $X^{*}(t)$ we can find an $L^{2}$-form $v$ on $X^{*}(t)$ which satisfies

$$
\begin{gathered}
u=d_{X^{*}(t)} v \\
\|v\|_{X^{*}(t)} \leqq C\|u\|_{X^{*}(t)}
\end{gathered}
$$

Here $d_{X^{*}(t)}$ means the exterior derivative on $X^{*}(t)$.

Proof. For an integer $q \geqq 1$, let us consider the following commutative diagram

$$
\begin{gathered}
V \stackrel{q^{K / 2}}{\longrightarrow} V \\
\downarrow \\
X^{*}(t) \stackrel{f_{q}}{\longleftarrow} X^{*}(q t) \\
\Psi \\
\Psi \\
\left(t, x^{\prime}, y\right) \leftarrow(q t, x, y),
\end{gathered}
$$

where $x^{\prime}=q x$. Note that $f_{q}$ is an isometry and preserves the fiber metrics between the bundle $V$ on $X^{*}(t)$ and $V$ on $X^{*}(q t)$. Moreover this commutes with the exterior derivative $d$. In fact, for $e \in V$ we find

$$
\begin{aligned}
d_{X^{*}(q t)}\left(q^{K / 2} e\right) & =\sum_{j} d x_{j} \otimes N_{j} q^{K / 2} e \\
& =q^{K / 2} \sum_{j} q d x_{j} \otimes N_{j} e \\
& =q^{K / 2} \sum_{j} d x_{j}^{\prime} \otimes N_{j} e \\
& =q^{K / 2} d_{X^{*}(t)} e
\end{aligned}
$$

because the commutation relation (5.8.4) implies 


$$
N_{j} q^{K / 2}=q^{K / 2+1} N_{j} \quad(j=1, \cdots, n)
$$

Now, for each $t(0<t<2)$, we can find a positive integer $q$ such that $1<q t<3$. Then, by the Hodge-Kodaira decomposition (Eemma 5.9.1), we can find a unique $v$ which satisfies the following;

$$
f_{q}^{*} u_{i}=d \tilde{v}
$$

(5.10.10) $v$ is orthogonal to the space of Larmonic forms and $R\left(d^{*}\right)$, the range of the adjoint operator of (the weak closure of) $d$.

Then $\tilde{v}$ satisfies for some constant $\mathbb{C}$ (independent of $t$ )

$$
\|\tilde{\nabla}\|_{X^{*}(q t)} \leqq C\left\|f^{*} u\right\|_{X^{*}(q t)} .
$$

Since $\tilde{v}$ is unique, $v$ is stable under the covering transformation of $f_{q^{\circ}}$ There. fore we can find $v$ such that $\widetilde{v}=f_{q}^{*} v$. Then

$$
u=d v .
$$

Furthermore we have

$$
\left\|f^{*} u\right\|_{X^{*}(q t)}=q^{n}\|u\|_{X^{*}(t)}
$$

and

$$
\left\|f^{*} v\right\|_{X^{\prime}(q t)}=q^{n}\|v\|_{X^{\prime}(t)}
$$

Hence (5.10.11) implies

$$
\|v\|_{X^{4}(t)} \leqq \mathbb{C}\|u\|_{X^{\prime}(t)}
$$

where $\mathbb{C}$ is independent of $t$.

Q.E.D.

Lemmm 5.10.3。 Let $A$ be the operator given in Definition 5.8.4, and let $h$ be a square-integrable harmonic form on $X^{*}(t)$. Then we find

$$
L_{\partial / \partial x_{j}} h=0 \quad \text { for any } j
$$

and

Ah is harmonic.

Here $L_{\partial / \partial x_{j}}$ is the Lie derivative of $\partial / \partial x_{j}$ acting on $V$ irivially.

Proof. Let us first recall the following well-known fact:

Sublemarima 5.10.4. Let $G$ be a connected iopological group acting on a topological space $X, F^{\circ}$ a complex of sheaves on $X$ (or objects in the derived category). Lei $f: G \times X \rightarrow X$ be the composition map and let $p: G \times X \rightarrow X$ be the 
projection. Let $\alpha$ be an isomorphism $f^{-1} F^{\circ} \underset{\rightarrow}{\rightarrow} p^{-1} F^{\circ}$. For any $g$ in $G$ let $i_{g} \circ \alpha: g^{-1} F^{\circ} \underset{\rightarrow}{\rightarrow} F^{\circ}$ be the morphism given by $i_{g}: X \rightarrow G \times X$. Then we find

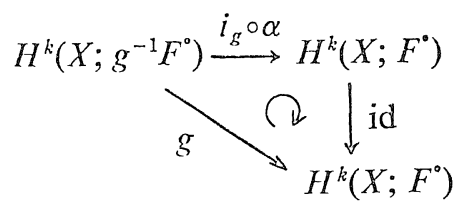

commutes.

Now we use this result in the following manner: Take $G=\mathbb{R}^{n}$ and let $G$ act on $X^{*}(t)$ by $a:(x, y) \mapsto(x+a, y)$, which can be extended to the action on ${ }^{\pi} H$ so that it acts on $V$ by $e^{\Sigma a_{j} N_{j}}$. Therefore this action is trivial on the cohomology groups $H^{k}\left(X^{*}(t) ;\left.{ }^{\pi} H\right|_{X^{*}(t)}\right)$. In particular, for $a \in \mathbb{Z}^{n}$, this action is nothing but $e^{\Sigma a_{j} N_{j}}$ on ${ }^{\pi} H$. This means that the actions of $N_{j}$ on $H^{k}\left(X^{*}(t)\right.$; $\left.\left.{ }^{\pi} H\right|_{X^{r}(t)}\right)$ is trivial. Therefore for $a \in \mathbb{R}^{n}$, the action of $a$ on $X^{*}(t)$ and the trivial action on $V$ induce the trivial action on the cohomology groups. Now, by the induction hypothesis, the cohomology group is isomorphic to the space of square-integrable harmonic forms. This implies $h(x+a)=h(x)$ for any square-integrable harmonic form $h$. Hence $L_{\partial / \partial_{x_{j}}} h=0$ holds.

The assertion (5.10.17) is an immediate consequence of the commutation relations

$$
\left[A, d_{X^{*}(t)}\right]=2 d_{x},\left[A, d^{*}{ }_{X^{+}(t)}\right]=2 \sum_{j}\left(t^{2} y_{j}^{2}\right)^{-1} i_{\partial / \partial x_{j}} L_{\partial / \partial x_{j}} .
$$

proved in Lemma 5.8.4, and the fact that a harmonic form $h$ satisfies $d_{X^{*}(t)} h=$ $d_{X}^{*}(t) h=0$.

Q.E.D.

Corollary 5.10.5. Let us identify $V$-valued forms on $X^{*}(t)$ and those on $X^{*}\left(t^{\prime}\right)$ by $(t, x, y) \leftrightarrow\left(t^{\prime}, x, y\right)$. Then a square-integrable harmonic form $h$ on $X^{*}(t)$ is also harmonic on $X^{*}\left(t^{\prime}\right)$.

Proof. Let $\widetilde{X^{*}\left(t^{\prime}\right)}$ be the universal covering of $X^{*}(t)$. Then we have the following commutative diagram:

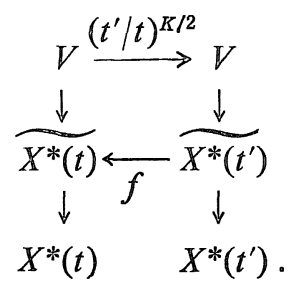


Here $f$ is given by $\left(t^{\prime}, x^{\prime}, y\right) \mapsto(i, x, y)$ with $x^{\prime}=\frac{\dot{i}}{!^{\prime}} x$. IILEn $f^{\prime}$ and $\left(i^{\prime} / i^{\prime K / 2}\right.$ give isomorphisms of Riemannian nuanifolds and vector bundles witl Hermitian metric. Hence the Laplacians $\Delta_{t}$ and $\Delta_{t^{\prime}}$ coincide. Therefore the resmit follows from the fact that if $h$ is harmonic then $\left(f^{\prime} / 2\right)^{1 / 2} / h$ is also haresoric.

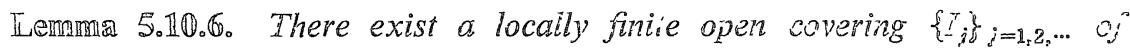
$\mathbb{R}^{+}(=\{t \in \mathbb{R} ; i>0\})$ and an associaied paritition of unity $\left\{a_{j}\right\}_{j}=1,2, \ldots$ which scitisfy the following conditions for some constanis a and $c$ :

(5.10.18) If $\hat{t}_{1}$ and $i_{2}$ belong to $I_{j}$, then $\left|i_{1} / t_{2}\right| \leqq a$.

(5.10.19) $a_{j}$ is a non-negative $\mathbb{C}^{\infty}{ }^{\infty}$ function with its suppors $\mathrm{inh}_{j_{j}}$.

(5.10.20) $\sum_{j} a_{j}=1$.

(5.10.21) $\sum\left|t \frac{\partial a_{j}}{\partial t}\right| \leqq c$.

Proof. Let $J_{l}(l \in \mathbb{Z})$ denote an open incerval $\{s \in \mathbb{Z} ; l-2<s<i+2\}$. Let $\chi(s)$ be a non-negative $\mathbb{C}^{\infty}$-function whose suppont is containec in $\{s \in \mathbb{R}$; ; $|s|<2\}$ and which is stricly positive on $\{s \in \mathbb{R} ;|s|<1\}$. Let $\chi_{m}(s)(m \in \mathbb{s})$ denote $\chi(s-m)$. Denote by $b_{l}(s)$ the function

$$
\frac{\chi_{l}(s)}{\sum_{m} \chi_{m}(s)} 。
$$

Then we immediately see:

$$
\begin{aligned}
& \text { supp } b_{l} \subset J_{l} \\
& \sum_{l} b_{l}=1
\end{aligned}
$$

Since $\sum_{l}\left|\frac{\partial}{\partial s} b_{l}(s)\right|$ is a periodic function, it musi be bounded on $\mathbb{R}$. Nexi define $\mathbb{I}_{l}$ and $a_{l}(t)$ by $\left\{t \in \mathbb{R}^{+} ; \log (l-2)<l<\log (l+2)\right\}$ and $b_{l}$ (log $\left.i\right)$, respectively. By renumbering $\mathbb{I}_{l}$ and $a_{l}$ suitably we can find the requireo $\bar{r}_{j}$ ano $a_{j}$.

C.E.I.

Let us now embark on the proof of Proposition 5.10.i. Let us fix a soint $\imath_{j}$ in each interval $\mathbb{I}_{j}$ in Lemma 5.10.6. Our strategy is to decompose closec $L^{2}$-form $\omega$ on $X^{*}$ in $\mathbb{L}_{(2)}^{k}\left(X^{*}\left(t_{j}\right) ;\left.H\right|_{X^{*}\left(i_{j}\right)}\right)$ first and then 0 paich their iogethe using the preceding lemmas. Let $u$ and $v$ be a form independent of di which satisfies 


$$
\omega=\frac{d t}{t} u+v
$$

Then the assumption that $\omega$ is a closed $\mathbb{L}^{2}$-form implies the following:

$$
\begin{gathered}
d_{X^{*}(t)} u=t \frac{\partial v}{\partial t} \\
d_{X^{*}(t)} v=0 \\
\int\|u(t)\|_{X^{*}(t)}^{2} \frac{d t}{t}<\infty \\
\int\|v(t)\|_{X^{*}(t)}^{2} \frac{d t}{t}<\infty .
\end{gathered}
$$

Here and in what follows we sometimes use the symbol $d_{X^{*}(t)}$ to emphasize that the exterior derivative is considered on $X^{*}(t)$. If there is no fear of confusion, we simply denote it by $d^{\prime}$.

In view of (5.10.26), we can decompose $v(t)$ as

$$
h_{j}(t)+d_{X^{*}(t)} w_{j}(t)
$$

with respect to the metric on $X^{*}\left(t_{j}\right)$, where $h_{j}(t)$ is a harmonic form. It is clear that $h_{j}(t)$ is independent of $j$, and hence we denote it simply by $h(t)$. By Lemma 5.10.2 we may assume without loss of generality

$$
\|h(t)\|_{X^{*}(t)} \leqq\|v(t)\|_{X^{*}(t)}
$$

and

$$
\left\|w_{j(t)}\right\|_{X^{*}(t)} \leqq C\|v(t)\|_{X^{*}(t)} .
$$

By the orthogonal decomposition in $L_{(2)}^{k}\left(X^{*}\left(t_{j}\right) ; H\right)$, we may further assume

(5.10.32) $w_{j}(t, x)$ is orthogonal to the space of closed forms in $L_{(2)}^{k}\left(X^{*}\left(t_{j}\right) ; H\right)$.

Let $\varphi(t)$ be a compactly supported $C^{\infty}$-function of $t \in \mathbb{R}$. Then it follows from $(5.10 .24),(5.10 .29)$ and $(5.10 .25)$ that

$$
\begin{aligned}
d^{\prime} \int \varphi(t) u(t) \frac{d t}{t} & =-\int\left(t \frac{\partial}{\partial t} \varphi(t)\right) v(t) \frac{d t}{t} \\
& =-\int\left(t \frac{\partial}{\partial t} \varphi(t)\right) h(t) \frac{d t}{t}-d^{\prime} \int\left(t \frac{\partial}{\partial t} \varphi(t)\right) w_{j}(t) \frac{d t}{t}
\end{aligned}
$$

Since $\int \frac{\partial}{\partial t}(\varphi(t)) h d t$ is harmonic it must be orthogonal to the space of coboundary forms, and hence this integral vanishes for any $\varphi$. Thus we obtain 


$$
\iota \frac{\partial h}{\partial t}=0
$$

and

$$
d^{\prime}\left(\int \varphi(t) u(t) \frac{d t}{t}+\int\left(\hat{t} \frac{\partial}{\partial t} \varphi(\hat{t})\right) w_{j} \frac{d t}{t}\right)=0
$$

Next let us decompose $u(t)$ in $L_{(2)}^{k}\left(\mathbb{X}^{*}\left(\hat{t}_{j}\right) ; H\right)$ as

$$
u(t)=h^{1}(t)+\beta_{j}(t)+d^{\prime} \alpha_{j}(t),
$$

where $h^{1}(t)$ is the harmonic part of $u(t)$ (independent of $j$ ) and $\beta_{j}(t)$ is orthogonal to the space of closed forms. Then we have

$$
\left\|h^{1}(t)\right\|_{X^{*}\left(i_{j}\right)} \leqq\|u(t)\|_{X^{*}\left(t_{j}\right)}
$$

and

$$
\left\|\beta_{j}(i)\right\|_{X^{*}\left(t_{j}\right)} \leqq\|u(i)\|_{X^{*}\left(i_{j}\right)}
$$

Note that (5.10.37) implies

$$
\left\|h^{1}(t)\right\|_{X^{*}(t)} \leqq \mathbb{C}\|u(t)\|_{X^{*}(t)} \quad \text { for } \quad t \in \mathbb{I}_{j}
$$

with a constant $C$ independent of $j$. Since $h^{1}(\hat{t})$ is a globally defined form it means

$$
\left\|h^{1}(t)\right\|_{X^{*}(t)} \leqq \mathbb{C}\|u(t)\|_{X^{*}(t)}
$$

with a constant $\mathbb{C}$ independent of $t$. It also follows from $(5.10 .36)$ that for any compactly supported $C^{\infty}$-function $\varphi(\hat{t})$ we find

$$
\begin{gathered}
\int\left(\varphi(t) u(t)+\left(t \frac{\partial}{\partial t} \varphi(t)\right) w_{j}(t)\right) \frac{d i}{t} \\
=\int \varphi(i) h^{1}(t) \frac{d t}{t}+\int\left(\varphi(t) \beta_{j}+\left(\hat{\imath} \frac{\partial}{\partial t} \varphi(\hat{t})\right) w_{j}(t)\right) \frac{d \hat{t}}{\hat{t}} \\
+\int \varphi(t) d^{\prime} \alpha_{j}(t) \frac{d t}{\imath}
\end{gathered}
$$

Since the left hand side together with the first and the third terms in the right side of (5.10.40) is closed, the deinnition of $\beta_{j}$ and the property (5.10.32) imply

$$
\beta_{j}=\hat{\imath} \frac{\partial w_{j}}{\partial t}
$$

Hence (5.10.38) implies

$$
\left\|\hat{i} \frac{\partial w_{j}}{\partial i}(t)\right\|_{X^{*}\left(t_{j}\right)} \leqq\|u(i)\|_{X^{*}\left(i_{j}\right)} .
$$


In view of the structure of the metrics and the condition (5.10.18) on the size of the interval $I_{j}$, we can find a constant $C$ independent of $j$ such that

$$
\left\|t \frac{\partial w_{j}}{\partial t}\right\|_{X^{*}(t)} \leqq C\|u\|_{X^{1}(t)} \quad \text { for } \quad t \text { in } I_{j}
$$

Let $w_{j k}$ denote $w_{j}-w_{k}$. Then it follows from the definition (5.10.29) that $w_{j k}$ is a closed form on $I_{j} \cap I_{k}$. Furthermore we have

$$
\left\|w_{j k}(t)\right\|_{X^{*}(t)} \leqq C\|v(t)\|_{X^{*}(t)}
$$

and

$$
\left\|t \frac{\partial w_{j_{k}}}{\partial t}(t)\right\|_{X^{*}(t)} \leqq C\|u(t)\|_{X^{*}(t)} \quad \text { for } \quad t \text { in } \mathbb{I}_{j} \cap \mathbb{I}_{k}
$$

Using the partition of unity $\left\{a_{j}\right\}$ introduced in Lemma 5.10.6, we define a closed form $\gamma_{j}$ on $I_{j}$ by

$$
\sum_{k} a_{k} w_{j k}
$$

Then we obtain from Lemma 5.10.6, (5.10.44) and (5.10.45) the following:

$$
\begin{gathered}
\left\|r_{j}(t)\right\|_{X^{*}(t)} \leqq C\|v(t)\|_{X^{*}(t)} \quad\left(t \in I_{j}\right) \\
\left\|t \frac{\partial \gamma_{j}}{\partial t}(t)\right\|_{X^{*}(t)}=\left\|\left(\sum_{k} t \frac{\partial a_{k}}{\partial t} w_{j k}+\sum_{k} a_{k} t \frac{\partial v_{j_{k}}}{\partial t}\right)\right\|_{X^{*}(t)} \\
\leqq C^{\prime}\left(\|v(t)\|_{X^{*}(t)}+\|u(t)\|_{X^{*}(t)}\right) \quad\left(t \in I_{j}\right)
\end{gathered}
$$

where $C^{\prime}$ is another constant independent of $j$.

On the other hand, it follows from the definition of $w_{j k}$ and $r_{j}$ that

$$
\begin{aligned}
\gamma_{j}-\gamma_{k} & =\sum_{l} a_{l}\left(w_{j}-w_{l}\right)-\sum_{l} a_{l}\left(w_{k}-w_{l}\right) \\
& =w_{j}-w_{k} \quad\left(t \in I_{j} \cap I_{k}\right) .
\end{aligned}
$$

Set $w=w_{j}-r_{j}$. Then (5.10.49) guarantees that $w$ is a globally defined form, and (5.10.31) and (5.10.43) imply

$$
\begin{gathered}
\|w(t)\|_{X^{*}(t)} \leqq C\|v(t)\|_{X^{*}(t)}, \\
\left\|t \frac{\partial w}{\partial t}\right\|_{X^{*}(t)} \leqq C\left(\|v(t)\|_{X^{*}(t)}+\|u(t)\|_{X^{*}(t)}\right)
\end{gathered}
$$

for some constant $C$ independent of $t$. Combining (5.10.36), (5.10.41) and the definition of $w$, we obtain

$$
u-t \frac{\partial w}{\partial t}=h^{1}+t \frac{\partial r_{k}}{\partial t}+d^{\prime} \alpha_{k}
$$


where $\gamma_{k}$ is a closed form on $\mathbb{I}_{k}$. Hence $u-t \frac{\partial w}{\partial t}$ is a closed form. By (5.10.51), (5.10.39), (5.10.27) and (5.10.28), it is a square-integrable form on $X^{*}$. Therefore, by the same reasoning as in the proof of Lemma 5.10.2, we find $\alpha(t)$ and a harmonic form $h^{2}(t)$ which satisfy

$$
\left\{\begin{array}{l}
u-t \frac{\partial w}{\partial t}=h^{2}+d^{\prime} \alpha \\
\left.\|\alpha(t)\|\right|_{X^{*}(t)} \leqq C\left\|u-t \frac{\partial w}{\partial t}\right\|_{X^{*}(t)} \leqq C^{\prime}\left(\|u\|_{X^{*}(t)}+\|v\|_{X^{*}(t)}\right) \\
\left\|h^{2}(t)\right\|_{X *(t)} \leqq C^{\prime}\left(\|u\|_{X^{*}(t)}+\|v\|_{X^{*}(t)}\right)
\end{array}\right.
$$

with some constants $C$ and $C^{\prime}$ which are independent of $t$. Hence (5.10.53) combined with (5.10.27) and (5.10.28) entails that $\alpha$ and $h^{2}$ are square-integrable form on $X^{*}$. Then it follows from the definition of $u, v$ and $w$ that

$$
\begin{aligned}
\omega & =\frac{d t}{t} u+v \\
& =\frac{d t}{t}\left(t \frac{\partial w}{\partial t}+h^{2}+d^{\prime} \alpha\right)+h+d^{\prime} w_{j} \\
& =d t \frac{\partial w}{\partial t}+\frac{d t}{t} h^{2}+d^{\prime}\left(\frac{d t}{t} \alpha\right)+h+d^{\prime} w \\
& =d_{X} *\left(w+\frac{d t}{t} \alpha\right)+\frac{d t}{t} h^{2}+h .
\end{aligned}
$$

Since $w+\frac{d t}{t} \alpha$ is square-integrable by (5.10.50) and (5.10.53), the relations (5.10.54) and (5.10.34) are exactly the same as what Proposition 5.10 .1 asserts. Thus we have completed the proof of Proposition 5.10.1 at long last.

The following proposition gives us a characterization of an $L^{2}$-coboundary form on $X^{*}$. Combining Proposition 5.10.1 and the following one, we have complete description of the $L^{2}$-cohomology groups in terms of $L^{2}$-harmonic forms.

Proposition 5.10.7. Let $h$ and $h^{1}$ be the harmonic forms given in Proposition 5.10.1. Then the following two statements are equivalent:

(5.10.55) $h+\frac{d t}{t} h^{1}$ is a coboundary of an $L^{2}$-form on $X^{*}$. 
(5.10.56) (i) $h=0$

and

(ii) There exists a harmonic form $H^{1}$ which is square-integrable on $X^{*}$ and satisfies $h^{1}=t \frac{\partial H^{1}}{\partial t}$.

Proof. It is clear that (5.10.56) implies (5.10.55). Let us show that (5.10.55) implies (5.10.56). Let $u$ and $v$ be forms free from $d t$ and suppose that they satisfy

$$
h+\frac{d t}{t} h^{1}=d_{X^{*}}\left(\frac{d t}{t} u+v\right) .
$$

Since

$$
d_{X^{*}}\left(\frac{d t}{t} u+v\right)=\frac{d t}{t}\left(-d^{\prime} u+t \frac{\partial v}{\partial t}\right)+d^{\prime} v
$$

we find

$$
h=d^{\prime} v
$$

and

$$
h^{1}=t \frac{\partial v}{\partial t}-d^{\prime} u
$$

Since $h$ is a harmonic form, (5.10.59) implies

$$
h=d^{\prime} v=0 \text {. }
$$

Hence, by the same reasoning as in the proof of Proposition 5.10.1, we can find a square-integrable harmonic form $H^{1}$ and a square-integrable form $w$ which satisfy

$$
v=H^{1}+d^{\prime} w .
$$

Then (5.10.60) and (5.10.62) imply

$$
h^{1}-t \frac{\partial H^{1}}{\partial t}=d^{\prime}\left(t \frac{\partial w}{\partial t}-u\right)
$$

Hence we conclude

$$
h^{1}=t \frac{\partial H^{1}}{\partial t}
$$

This completes the proof of Proposition 5.10.7.

§.11。 By the induction hypothesis, each cohomology class in $\mathbb{H}^{k}\left(X^{*}(1)\right.$; 
$\left.\left.{ }^{\pi} H\right|_{X^{*}(1)}\right)$ can be represented by a harmonic form $h$ on $\pi^{*}(1)$, while Lemma 5.10.3 guarantees that $A h$ is also harmonic. Thus the operator $A$ defines an endomorphism of $H^{k}\left(X^{*}(1) ;\left.{ }^{\pi} H\right|_{X^{*}(1)}\right)$. As we shall prove in a later subscction ( $\$ 5.13)$, the purity theorem gives a bound on the eigenvalues $\lambda$ of the operator $A$ acting on $H^{k}\left(X^{*}(1) ;\left.{ }^{\pi} H\right|_{X^{*}(1)}\right)$ as follows:

Propositiom 5.11.1. Let $\lambda$ be an eigenvalue of $A$ acting on the space of square-integrable harmonic $k$-forms on $\left.\mathbb{H}\right|_{X^{*}(1)}$. Then w'e find:

$$
\begin{cases}\lambda \leqq k, & \text { if } k<n \\ \lambda \geqq k+1, & \text { if } k \geqq n .\end{cases}
$$

§5.12. Admitting Proposition 5.11.1 for a while, let us show how Theorem 5.4.1 follows from it. We begin our discussion by recalling the following celebrated inequality due to Hardy $([\mathbb{H}-\mathbb{L}-\mathbb{P}]$, p. 245, 339):

Lemrma 5.12.1. Let $r$ be a real number different from 0 . Let $f(i)$ be a measurable function such that $\int_{0}^{\infty} t^{r}|f|^{2} \frac{d t}{t}$ is jinite. Then we can find $\mathbb{F}(t)$ which satisfies the following:

$$
\begin{gathered}
\hat{t} \frac{d F}{d t}=f_{0} \\
\int_{0}^{\infty} t^{r}|\mathbb{F}|^{2} \frac{d t}{t} \leqq\left(\frac{2}{r}\right)^{2} \int_{0}^{\infty} t^{r}\left|t^{r}\right|^{2} \frac{d_{t}^{t} t}{t} .
\end{gathered}
$$

Using this lemma, we claim that $h^{1}$ in Proposition 5.10 .1 may be supposed to be 0 . In fact, we may assume without loss of generality that $h^{1}$ is an eigenfunction of $A$ with the eigenvalue $\lambda$. By (5.3.12), we then find

$$
\left\|h^{1}\right\|_{X^{*}}^{2}=\int t^{n-\lambda}\left\|h^{1}\right\|_{X^{*}(1)}^{2} \frac{d t}{t} .
$$

Since Proposition 5.11.1 guarantees $n-\lambda \neq 0$, we can find by Lemma 5.12.1 a square-integrable harmonic form $H^{1}$ such that

$$
t \frac{\partial H^{1}}{\partial \hat{\imath}}=h^{1}
$$

Since $\mathbb{H}^{1}$ is harmonic, Lemma 5.10 .3 and $(5.12 .3)$ imply

$$
d_{X} H^{1}=\frac{d t}{t} h^{1}
$$

that is, $\frac{d t}{t} h^{1}$ is an exact $L^{2}$-form on $X^{*}$. Thus we may suppose fiom the 
first that $h^{1}=0$.

Next let us discuss $h$ in Proposition 5.10.1. Again we may suppose that $h$ is an eigenfunction of $A$ with the eigenvalue $\lambda$. Then $\|h\|_{X^{*}}^{2}=\int t^{n-\lambda}\|h\|_{X^{*}(1)}^{2} d t / t$, and hence (5.10.3) implies that $h$ should vanish if it is an $L^{2}$-form on $X^{*}$, in case $\lambda \geqq n$. Therefore Proposition 5.11.1 implies $h=0$ if $k \geqq n$. On the other hand, if $k<n$, then, again by Proposition 5.11.1, we find that the finiteness of $\|h\|_{X^{*}}$ is equivalent to the finiteness of $\|h\|_{X^{*}(1)}$.

Thus we have verified

$$
H^{k}\left(X ; \mathcal{L}^{\circ}(H)\right) \cong \begin{cases}0 & \text { if } k \geqq n \\ H^{k}\left(X \backslash\{0\} ;\left.\mathcal{L}^{\circ}(H)\right|_{X \backslash\{0\}}\right) & \text { if } k<n,\end{cases}
$$

and the induction proceeds.

$\S$ 5.13。 Now we shall prove Proposition 5.11.1. It will complete the proof of Theorem 5.4.1 at long last.

For a positive integer $q$, let us consider the diagram

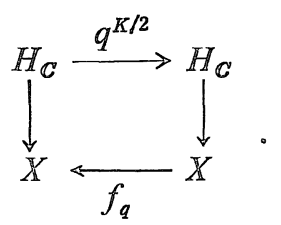

Here, $f_{q}$ is the morphism $\left(z_{1}, \cdots, z_{n}\right) \mapsto\left(z_{1}^{q}, \cdots, z_{n}^{q}\right)$. Then the morphism $q^{K / 2}$ : $H_{C} \rightarrow f_{*} H_{C}$ extends to

$$
r:{ }^{\pi} H_{\mathbb{C}} \rightarrow \mathbb{R} f_{*}{ }^{\pi} H_{C}
$$

Then, we obtain

$$
\begin{aligned}
\varphi_{q}^{(k)}: H^{k}\left(X \backslash\{0\} ;{ }^{\pi} H_{C}\right) & \stackrel{\gamma}{\simeq} H^{k}\left(X \backslash\{0\} ; \mathbb{R}^{\mathbb{R}} f_{*}{ }^{\pi} H_{C}\right) \\
& \simeq H^{k}\left(X \backslash\{0\} ;{ }^{\pi} H_{C}\right) .
\end{aligned}
$$

Since $H^{k}\left(X \backslash\{0\} ;{ }^{\pi} H_{C}\right) \simeq H^{k}\left(X(1) ;{ }^{\pi} H_{C}\right) \simeq H_{(2)}^{h}\left(X^{*}(1) ; H\right)$ and since this space is represented by the space $h^{k}$ of harmonic forms, $\varphi_{q}^{(k)}$ is represented by $q^{A / 2}$ on $h^{k}$. Therefore in order to show Proposition 5.11.1, it suffices to show that any eigenvalue $\mu$ of $\varphi_{q}^{(k)}$ satisfies

$$
\begin{array}{ll}
\mu \leqq q^{k / 2} & \text { if } \quad k<n \\
\mu \geqq q^{(k+1) / 2} & \text { if } \quad k \geqq n .
\end{array}
$$

Let us first consider the case where $k<n$. Then 


$$
H^{k}\left(X ;{ }^{\pi} H_{C}\right) \underset{\sim}{\rightarrow} H^{k}\left(X \backslash\{0\} ;{ }^{\pi} H_{C}\right)
$$

holds. Hence $\varphi_{q}^{(k)}$ defines an endomorphism of $H^{k}\left(X ;{ }^{\pi} H_{C}\right)$. We shall show the required bound on the eigenvalues of $\varphi_{q}^{(k)}$ follows from the purity theorem (Theorem 4.0.1.). For this purpose, we may, and do, enlarge $X$ to $\mathbb{C}^{n}$. Let $\mathscr{M}$ be the $\mathscr{D}_{X}$-module corresponding to ${ }^{\pi} H_{C}$, that is,

$$
\operatorname{DR}(\mathscr{M}) \simeq{ }^{\pi} H_{\mathbb{C}}
$$

Then the homomorphism $\gamma$ extends to

$$
r: \operatorname{DR}(\mathscr{M}) \rightarrow f_{*} \operatorname{DR}(\mathscr{M})
$$

given by $\omega \otimes e \mapsto f^{*} \omega \otimes q^{K / 2} e$ for $\omega \in \Omega(* Y)$ and $e \in V$. Now we can calculate $H^{k}\left(X ;{ }^{\pi} H_{C}\right)$ by $H^{k}\left(\Pi\left(N_{1}, \cdots, N_{n}\right)\right)$, because $\Gamma(X ; \mathrm{D} \mathbb{R}(\mathscr{M}))$ is quasi-isomorphic to the partial Koszul complex $\Pi\left(N_{1}, \cdots, N_{n}\right)$. Then, on $\Pi\left(N_{1}, \cdots, N_{n}\right)$, the homomorphism $r$ is given by

$$
r: \operatorname{Im} N_{j_{1}} \cdots N_{j_{\nu}} \ni e \mapsto q^{K / 2+v} e .
$$

If we endow $\Pi\left(N_{1}, \cdots, N_{n}\right)$ with the mixed $\mathbb{H}$ lodge structure, then $K$ acts on $\operatorname{Gr}_{\mu}^{W}\left(\Pi\left(N_{1}, \cdots, N_{n}\right)^{k}\right)$ by $\mu-w-2 k$, and hence $\gamma$ acts by $q^{\frac{1}{2}(\mu-w-2 k)+k}=q^{\frac{1}{2}(\mu-w)}$. Now the purity theorem says

$$
\operatorname{Gr}_{\mu}^{W}\left(H^{k}\left(\Pi\left(N_{1}, \cdots, N_{n}\right)\right)\right)=0 \quad \text { for } \quad \mu>w+k .
$$

Thus any eigenvalue $\mu$ of $\varphi_{q}^{(k)}$ satisfies

$$
\mu \leqq q^{\frac{1}{2}((w+k)-w)}=q^{\frac{1}{2} k}
$$

Let us now consider the case where $k \geqq n$. In view of the long exact sequence for local cohomology groups, we obtain

$$
H^{k}\left(X \backslash\{0\} ;{ }^{\pi} H\right) \cong H_{\{0\}}^{k+1}\left(X ;{ }^{\pi} H\right) \quad(k \geqq n) .
$$

On the other hand, the Poincaré-Verdier duality implies

$$
\left.H_{\{0\}}^{k+1}\left(X ;{ }^{\pi} H\right) \cong\left(H^{2 n-1-k}\left(X ;{ }^{\pi} H\right)^{*}\right)\right)^{\prime} .
$$

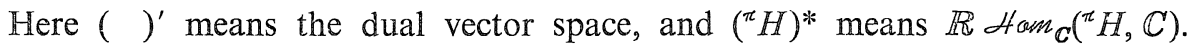
Since $f$ is a finite-covering map, $\left(f_{*}^{\pi} H^{*}\right)^{*} \cong f_{*}\left(\left({ }^{\pi} H\right)^{*}\right)$ holds. Let $\alpha^{*}$ denote the dual morphism of $\alpha:{ }^{\pi} H_{\mathbb{C}} \rightarrow \mathbb{R} f_{*}\left({ }^{\pi} H_{C}\right) \cong{ }^{\pi}\left(f_{*} H_{C}\right)$. If we denote by $\beta$ the map from ${ }^{\pi}\left(H_{C}^{*}\right)$ to $f_{*}{ }^{\pi}\left(H_{\mathbb{C}}^{*}\right)$ obtained in the same manner as in the definition of $\alpha$, we find

$$
\alpha^{*} \circ \beta=q^{n}, \quad \text { the number of } \int^{-1}(z) \text { for a generic } z \text {. }
$$


In fact, since $\operatorname{Hom}\left({ }^{\pi} H_{C},{ }^{\pi} H_{C}\right)=\operatorname{Hom}\left(\left.{ }^{\pi} H_{C}\right|_{X^{*}},\left.{ }^{\pi} H_{C}\right|_{X^{*}}\right)$, it is enough to prove (5.13.8) on $X^{*}$. This can be checked easily.

Passing to the level of the cohomology groups, we conclude the following claim (5.13.9) from (5.13.8).

$$
\begin{aligned}
& \text { The endomorphism } H^{2 n-k-1}\left(\alpha^{*}\right) \circ H^{2 n-k-1}(\beta) \text { of } \\
& H^{2 n-k-1}\left(X ;{ }^{\pi} H^{*}\right) \text { coincides with } q^{n} \text {. }
\end{aligned}
$$

As $k \geqq n$ in the present situation, we have $2 n-k-1<n$. Hence any eigenvalue of $H^{2 n-k-1}(\beta)$ should be equal to or smaller than $q^{(2 n-k-1) / 2}$. Therefore it follows from (5.13.9) that any eigenvalue of $H^{2 n-k-1}\left(\alpha^{*}\right)$ is equal to or bigger than $q^{(k+1) / 2}$. Since $H^{2 n-k-1}\left(\alpha^{*}\right)=H_{\{0\}}^{k}(\alpha)^{*}=\varphi_{q}^{(k) *}$, the eigenvalues of $\varphi_{q}^{(k)}$ enjoy the same property. Thus we have completed the proof of Proposition 5.11.1.

\section{§ 6. The Hodge Decomposition and the Hard Lefschetz Theorem}

$\S 6.0$. In the preceding sections, we showed that when $X$ is a compact complex manifold, the $L^{2}$-cohomology groups give the intermediate cohomology groups of the variation of polarized Hodge structure given on the complement of a normally crossing hypersurface in $X$.

Now we shall show that, when $X$ is a Kähler manifold, the decomposition theorem of Hodge, Weyl, Kodaira, ... for the constant case still hold for the variation of polarized Hodge structure.

Moreover, as observed in $[\mathbb{Z}]$, the results of Hodge (e.g. [W]) hold in this case also. Referring to $[\mathrm{Z}]$ and $[\mathrm{W}]$ for the proofs, we shall just state the facts that we need in our context.

$\S \overline{6}_{0} \mathrm{H}_{\text {. }}$ Let $X$ be a Kähler manifold with a Kähler metric $\omega$. Using a local coordinate $z_{j}$ of $X, \omega$ has the form

$$
\omega=\frac{\sqrt{-1}}{2} \sum_{\alpha, \beta} h_{\alpha \beta} d z_{\alpha} d \bar{z}_{\beta}
$$

with a positive definite Hermitian form $\left(h_{\alpha \beta}\right)_{\alpha, \beta}$. We confuse this $\mathbb{K}$ ähler form with a Riemannian metric $\sum h_{\alpha \beta} d z_{\alpha} \otimes d \bar{z}_{\beta}$. Let $H$ be a variation of polarized Hodge structure of weight $w$. If we denote by $\mathcal{E}$ the sheaf of $C^{\infty}$-functions, $\mathcal{E}(H)=\mathcal{E} \bigotimes_{C} H_{C}$ has also a Hodge decomposition by

$$
\mathcal{E}(H)=\bigoplus_{p, q=w} \mathcal{H}^{p, q}
$$

Here $\mathcal{H}^{p, q}=\underset{\mathcal{O}_{\bar{X}}}{\mathcal{E} F^{p}}(H) \cap \mathcal{E} \bigotimes_{\mathcal{O}_{\bar{X}}} \bar{F}^{q}(H)$. 
Denoting by $\mathcal{E}^{k}$ the sheaf of $\mathbb{C}^{\infty}-k$-forms and by $\mathcal{E}^{p, q}$ the sheaf of $\mathbb{C}^{\infty}-(p, q)$ forms, we define the filtration of $\mathcal{E}^{k}\left(H^{T}\right)=\mathcal{E}^{k} \otimes_{\mathcal{C}} H_{C}$ by

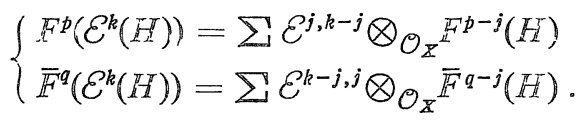

Then $\mathcal{E}^{k}(H)$ has also the Hodge decomposition.

$$
\mathcal{E}^{k}\left(H^{T}\right)=\bigoplus_{p+q=k+w} \mathscr{H}^{p, q}
$$

where $\mathcal{H}^{p, q}=\mathbb{F}^{p}\left(\mathcal{E}^{k}(H)\right) \cap{\overline{F^{q}}}^{q}\left(\mathcal{E}^{k}\left(\mathbb{H}^{i}\right)\right)$.

Now the exterior derivative $d$ of $\mathcal{E}^{\circ}$ extends to $\mathcal{E}^{\circ} \bigotimes_{\mathbb{C}} H_{\mathbb{C}}$ and satisfies

$$
\begin{aligned}
& d\left(F^{p}\left(\mathcal{E}^{k}(\mathbb{H})\right) \subset F^{p}\left(\mathcal{E}^{k+1}\left(\mathbb{F}^{I}\right)\right)\right. \\
& d\left(\bar{F}^{q}\left(\mathcal{E}^{k}\left(\mathbb{H}^{T}\right)\right)\right) \subset \bar{H}^{q}\left(\mathcal{E}^{k+1}(H)\right) \text {. }
\end{aligned}
$$

Therefore we have

$$
d \mathscr{H}^{p, q} \subset \mathscr{H}^{p+1, q} \oplus \mathscr{H}^{p, q+1} .
$$

We shall decompose $d: \mathcal{E}^{\circ}(H) \rightarrow \mathcal{E}^{\circ}(H)$ by $d=d^{\prime}+d^{\prime \prime}$ so that

$$
\begin{aligned}
& d^{\prime} \mathscr{H}^{p, q} \subset \mathscr{H}^{p+1, q} \\
& d^{\prime \prime} \mathscr{H}^{p, q} \subset \mathscr{H}^{p, q+1}
\end{aligned}
$$

Then we have

$$
\begin{aligned}
& d^{\prime} F^{p}\left(\mathcal{E}^{\circ}\left(H^{H}\right)\right) \subset \mathbb{F}^{p+1}\left(\mathcal{E}^{\circ}\left(H^{r}\right)\right), \\
& d^{\prime} \bar{F}^{q}\left(\mathcal{E}^{\circ}\left(H^{H}\right)\right) \subset \overline{\mathbb{F}}^{q}\left(\mathcal{E}^{\circ}(H)\right) \text {, } \\
& d^{\prime \prime} F^{p}\left(\mathcal{E}^{\circ}(H)\right) \subset \mathbb{R}^{p}\left(\mathcal{E}^{\circ}\left(H^{H}\right)\right) \text {, } \\
& d^{\prime \prime} \overline{\mathbb{F}}^{q}\left(\mathcal{E}^{\circ}\left(\mathscr{F}^{\top}\right)\right) \subset \overline{\mathbb{F}}^{q+1}\left(\mathcal{E}^{\circ}\left(H^{T}\right)\right) \text {. }
\end{aligned}
$$

Let us denote the Hermitian metric of the $\mathbb{C}^{\infty}$-vector bundle $\mathcal{E}^{\circ}(H)$ by $\langle\mid\rangle$. Then we can define for a linear differential operator $P$ acting on $\mathcal{E}^{\circ}(H)$ its formal adjoint $P^{*}$ by

$$
\int\left\langle P^{*} u \mid v\right\rangle \omega^{n}=\int\langle u \mid P v\rangle \omega^{n}
$$

for $u \in \mathscr{D} l^{\circ}(H)$ and $v \in \mathcal{E}^{\circ}(H)$ with compact support.

Following [N], let $L$ cienote the operator $u \mapsto \omega \wedge u$, and $\Lambda$ its formal adjoint. We denote by $d^{\prime *}, d^{\prime *}, d^{\prime \prime *}$ the formai adjoints of $d, d^{\prime}$ and $d^{\prime \prime}$ respectively. Then we have

$$
d^{*} \mathcal{H}^{p, q} \subset \mathcal{H}^{p-1, q} \oplus \mathcal{H}^{p, q-1}
$$




$$
\begin{aligned}
& d^{*} * \mathscr{H}^{p, q} \subset \mathscr{H}^{p-1, q}, \\
& d^{\prime \prime *} \mathcal{H}^{p, q} \subset \mathcal{H}^{p, q-1} .
\end{aligned}
$$

Then, as observed in $[\mathrm{W}]$ and $[\mathbb{Z}]$, we have

Proposition 6.1.1. (i) If we set $\Delta=d^{*} d+d d^{*}$, then $\Delta=2\left(d^{\prime *} d^{\prime}+d^{\prime} d^{\prime *}\right)$ $=2\left(d^{\prime \prime *} d^{\prime \prime}+d^{\prime \prime} d^{\prime \prime *}\right)$.

(ii) If $\alpha \in \mathcal{E}^{p}(H)(p \leqq n=\operatorname{dim} X)$ satisfies $L^{n-p+1} \alpha=0$, then $* \mathbb{C}_{0} \alpha=(-1)^{p(p-1) / 2} \times$ $\frac{1}{(n-p) !} L^{n-p} \alpha$. Here $*$ and $\mathbb{C}_{0}$ are the operators obtained by extending the *-operator and the Weil operator on $\mathcal{E}^{\circ}$ by tensoring $H_{C}$, respectively.

(iii) $d^{\prime} d^{\prime}=d^{\prime \prime} d^{\prime \prime}=0, \quad d^{\prime} d^{\prime \prime}+d^{\prime \prime} d^{\prime}=0, \quad d^{\prime *} d^{\prime \prime *}+d^{\prime \prime *} d^{\prime *}=0, d^{\prime *} d^{\prime \prime}+d^{\prime \prime} d^{\prime *}=0$, $d^{\prime \prime *} d^{\prime}+d^{\prime} d^{\prime \prime *}=0$.

(iv) $[L, d]=0, \quad\left[\Lambda, d^{*}\right]=0, \quad\left[L, d^{*}\right]=d^{\mathrm{C}}, \quad[\Lambda, d]=-d^{* \mathrm{C}}, \quad[L, \Delta]=0$.

Here $d^{\mathrm{C}}=C^{-1} d \mathrm{C}$ and $d^{* \mathrm{C}}=\mathbb{C}^{-1} d^{*} C$ and $\mathbb{C}$ is the Weil operator defined by $\left.\mathrm{C}\right|_{\mathscr{T}^{p, q}}=i^{p-q}$.

$\S 6.2$. Let $X$ be a compact $\mathbb{K a ̈ h l e r ~ m a n i f o l d ~ w i t h ~ a ~ K a ̈ h l e r ~ f o r m ~} \omega_{0}, Y$ a normally crossing hypersurface of $X$, and $H$ a variation of polarized Hodge structure on $X^{*}=X \backslash Y$. As in $[\mathbb{Z}]$, let us take a $C^{\infty}$-function $\varphi$ on $X^{*}$ with the following property: for any point $p$ of $Y$ take a local coordinate $\left(x_{1}, \cdots, x_{n}\right)$ such that $Y$ is given by $x_{1} \cdots x_{l}=0$. Then, for $\mathbb{C}^{\infty}$-functions $a_{j}$,

$$
\varphi=\sum_{j=1}^{l} \log \left(a_{j}-\log \left|z_{j}\right|^{2}\right)
$$

If we take $c>0$ sufficiently small, then $\omega=\omega_{0}+i c \partial \bar{\partial} \varphi$ is a Kähler metric with the desired behavior

$$
\omega \sim i \sum_{j \leqq l} \frac{d z_{j} d \bar{z}_{j}}{\left(\left|z_{j}\right| \log \left|z_{j}\right|\right)^{2}}+i \sum_{j>l} d z_{j} d \bar{z}_{j} .
$$

Therefore $\left(X^{*}, \omega\right)$ is a complete Riemannian manifold. As in $\S 5.3$, let us denote by $L_{(2)}^{k}\left(X^{*} ; H\right)$ the space of square integrable $k$-forms with value in $H_{C}$ with the norm $\langle\langle f \mid f\rangle\rangle=\int\langle f \mid f\rangle \omega^{n}$. Since $\mathcal{E}^{k} \otimes H_{\mathbb{C}}=\bigoplus_{p+q=k+w} \mathcal{H}^{p, q}$ is the orthogonal decomposition, $L_{(2)}^{k}\left(X^{*}, H\right)$ decomposes

$$
L_{(2)}^{k}\left(X^{*} ; H\right)=\bigoplus_{p+q=k+w} L_{(2)}^{p, q}\left(X^{*} ; H\right)
$$

Here $L_{(2)}^{p, q}\left(X^{*} ; H\right)$ is the space of square integrable sections of the bundle $\mathcal{H}^{p, q}$. The (6.2.1) is the decomposition of Hilbert spaces.

Set 


$$
F^{p}\left(L_{(2)}^{k}\left(X^{*} ; H\right)\right)=\bigoplus_{\substack{p^{\prime}+q^{\prime}=k+w \\ p^{\prime} \geqq p}} L_{(2)}^{p^{\prime} q^{\prime}}\left(X^{*} ; H\right)
$$

and define $\bar{F}^{p}\left(L_{(2)}^{k}\left(X^{*} ; H\right)\right)$ similarly. Then, with these filtrations, $L_{(2)}^{k}\left(X^{*} ; H\right)$ has a Hodge structure of weight $k+w$.

In what follows, we simply denote by $d$ and $d^{*}$ the weak closure of them, that is,

$D(d)=\left\{u \in L_{(2)}^{k}\left(X^{*} ; H\right) ; d u\right.$ in the distribution sense belongs to $\left.L_{(2)}^{k+1}\left(X^{*} ; H\right)\right\}$, $D\left(d^{*}\right)=\left\{u \in L_{(2)}^{k}\left(X^{*} ; H^{*}\right) ; d^{*} u\right.$ in the distribution sense belongs to $\left.L_{(2)}^{k-1}\left(X^{*} ; H\right)\right\}$ 。

Since $\omega$ is a complete metric, it is classically well-known that the strong closure of $d$ and the weak closure of $d$ coincide In particular, $d^{*}$ is the (operator-theoretic) adjoint of $d$. (See Appendix, Theorem A.4.1.)

Let us define $\Delta$ by specifying its domain as follows;

$$
D(\Delta)=\left\{u \in D(d) \cap D\left(d^{*}\right) ; d u \in D\left(d^{*}\right), d^{*} u \in D(d)\right\} .
$$

Then, again by the completeness of the metric, $\Delta$ thus defined coincides with the veak closure of $\Delta$, i.e., the operator whose domain is given by $\left\{u \in L_{(2)}^{k}\left(X^{k} ; H\right) ; \Delta u\right.$ in the distribution sense belongs to $\left.L_{(2)}^{k}\left(X^{*} ; H^{\prime}\right)\right\}$, and it is self-adjoint. ([Ch], see also Appendix, Theorem A.4.1.)

Since the strong closure of $\Delta$ preserves the Hodge decomposition, we have

$$
D(\Delta)=\oplus D(\Delta) \cap L_{(2)}^{p, q}\left(X^{*} ; H\right) .
$$

Now our main result (Theorem 5.4.1) asserts

$$
H^{\circ}\left(X ;{ }^{\pi} H\right)=\operatorname{Ker} d / R(d),
$$

and this is finite-dimensional. Therefore $d$ has closed range. Hence we can use the result in $[\mathrm{Ko}]$ (cf. Theorem A.2.1 in Appendix). Denoting by $h^{k}$ the space of square-integrable harmonic $k$-forms, i.e., $h^{k}=\left\{u \in \mathbb{L}_{(2)}^{k}\left(X^{*} ; H\right)\right.$; $\Delta u=0\}$, we find

$$
h^{k} \cong H^{k}\left(X ;{ }^{\pi} H\right)
$$

In what follows, we denote by $H$ the orthogonal projection from $L_{(2)}^{k}\left(X^{*} ; H_{\overparen{C}}\right)$ to $h^{k}$, and by $G$ the Green operator (cf. Definition A.2.2 in Appendix); thus we have

$$
u=H(u)+\Delta G(u) \quad \text { for } \quad u \in L_{(2)}^{\circ}\left(X^{*} ; H_{C}\right) .
$$

Let $h^{p, q}$ denote $h^{k} \cap L_{(2)}^{p, q}\left(X^{*} ; H_{C}\right)$, where $p+q=k+w$. Since $\Delta$ preserves 
the Hodge decomposition, we then have

$$
\begin{aligned}
& h^{k}=\bigoplus_{p+q=k+w} h^{p, q}, \\
& H\left(L_{(2)}^{p, q}\left(X^{*} ; H\right)\right) \subset h^{p, q}, \\
& G\left(L_{(2)}^{p, q}\left(X^{*} ; H\right)\right) \subset L_{(2)}^{p, q}\left(X^{*} ; H\right) .
\end{aligned}
$$

We set $F^{p}\left(h^{k}\right)=\underset{\substack{p^{\prime}+q^{\prime}=k+w \\ p^{\prime} \geqq p}}{\bigoplus} h^{p^{\prime}+q^{\prime}}$ and define $\overline{\mathbb{F}}\left(h_{k}\right)$ similarly.

Now, we denote by $d^{\prime}$ and $d^{\prime \prime}$ their weak closures and $d^{\prime *}$ and $d^{\prime \prime *}$ their adjoint operators in the operator theoretic sense. Therefore $d^{\prime *}$ and $d^{\prime \prime *}$ are the strong closures. Although we can prove that their strong closure coincides with their weak closure, we do not use this fact in this paper. However, we use the fact that

$$
D^{\prime}(\Delta)=\left\{u \in D\left(d^{\prime}\right) \cap D\left(d^{\prime *}\right) ; d^{\prime} u \in D\left(d^{\prime *}\right) d^{\prime *} u \in D\left(d^{\prime}\right)\right\}
$$

and $D(\Delta)$ coincide.

In fact, $\left.\Delta\right|_{D^{\prime}(\Delta)}$ is a closed operator, because $\left.\Delta\right|_{D^{\prime}(\Delta)}=2\left(d^{\prime} d^{\prime *}+d^{\prime *} d^{\prime}\right)$. Since the weak closure and the strong closure of $\Delta$ coincide, we have

$$
D(\Delta)=D^{\prime}(\Delta)
$$

We have

$$
\begin{aligned}
& \text { If } u \in D\left(d^{\prime}\right) \text { satisfies } d^{\prime} u=0 \text {, then } d^{\prime} G(u)=0 \\
& \text { and } u=H(u)+2 d^{\prime} d^{\prime} * G(u) \text {. }
\end{aligned}
$$

In fact, we have $u=H(u)+\Delta G(u)$. Since $H(u) \in D(\Delta) \subset D\left(d^{\prime}\right)$, we have $\Delta G(u) \in D\left(d^{\prime}\right)$ and $d^{\prime} \Delta G(u)=0$. Therefore $2\left\langle d^{\prime *} d^{\prime} G(u) \mid d^{\prime *} d^{\prime} G(u)\right\rangle=\langle\Delta G(u)|$ $\left.d^{\prime *} d^{\prime} G(u)\right\rangle=\left\langle d^{\prime} \Delta G(u) \mid d^{\prime} G(u)\right\rangle=0$, which implies $d^{\prime *} d^{\prime} G(u)=0$. Then $\left\langle d^{\prime} G(u)\right|$ $\left.d^{\prime} G(u)\right\rangle=\left\langle d^{\prime *} d^{\prime} G(u) \mid G(u)\right\rangle=0$ implies $d^{\prime} G(u)=0$.

\section{Lemingma 6.2.1。 $a^{\prime \prime *} D(\Delta) \subset D\left(d^{\prime}\right)$.}

Proof. For $u \in D(\Delta) \subset D\left(d^{\prime \prime *}\right)$ let us take compactly supported $C^{\infty}$ sections $u_{n}$ such that $u_{n} \rightarrow u, \Delta u_{n} \rightarrow \Delta u$. It is enough to show that $d^{\prime} d^{\prime \prime *} u_{n}$ converges. Setting $v=u_{n}-u_{m}$, we have

$$
\begin{aligned}
& \left\langle\left\langle d^{\prime} d^{\prime \prime *} v \mid d^{\prime} d^{\prime \prime *} v\right\rangle\right\rangle \\
& =-\left\langle\left\langle d^{\prime \prime *} d^{\prime} v \mid d^{\prime} d^{\prime \prime *} v\right\rangle\right\rangle \\
& =-\left\langle\left\langle d^{\prime} v \mid d^{\prime \prime} d^{\prime} d^{\prime \prime *} v\right\rangle\right\rangle \\
& =\left\langle\left\langle d^{\prime} v \mid d^{\prime} d^{\prime \prime} d^{\prime \prime *} v\right\rangle\right\rangle \\
& =\left\langle\left\langle d^{\prime *} d^{\prime} v \mid d^{\prime \prime} d^{\prime \prime *} v\right\rangle\right\rangle .
\end{aligned}
$$


On the other hand,

$$
\left\|d^{\prime *} d^{\prime} v\right\|^{2}+\left\|d^{\prime} d^{\prime *} v\right\|^{2}=\left\|\left(d^{\prime *} d^{\prime}+d^{\prime} d^{\prime *}\right) v\right\|^{2}
$$

implies

$$
\left\|d^{\prime *} d^{\prime} v\right\| \leqq\|\Delta v\|
$$

Similarly we have

$$
\left\|d^{\prime \prime} d^{\prime \prime *} v\right\| \leqq\|\Delta v\|
$$

Thus we obtain $\left\|d^{\prime} d^{\prime \prime * v \mid} \mid \leqq\right\| \Delta v \|$. Hence

$$
\left\|d^{\prime} d^{\prime \prime *} u_{n}-d^{\prime} d^{\prime \prime *} u_{m}\right\| \rightarrow 0 \quad \text { if } \quad n, m \rightarrow \infty .
$$

Therefore $d^{\prime} d^{\prime \prime *} u_{n}$ converges.

Let us define the filtrations $F$ and $\bar{F}$ of $\mathcal{L}^{\circ}(H)$ by

$F^{p}\left(\mathcal{L}^{\circ}(H)\right)=\left\{u \in \mathcal{L}^{\circ}(H) ; u\right.$ is a distribution valued sections of $\left.F^{b}(\mathcal{E}(H))\right\}$ and similarly

$$
\bar{F}^{p}\left(\mathcal{L}^{\circ}(H)\right)
$$

Since $F^{p}\left(\mathcal{L}^{\circ}(H)\right)$ is an $\mathcal{E}$-module, $F^{p}\left(\mathcal{L}^{\circ}(H)\right)$ is a complex of soft sheaves. Then we have the following

$\mathbb{P}_{\text {roposition 6.2.2。 }} F^{p}\left(h^{k}\right) \rightarrow H^{k}\left(X ; F^{p}\left(\mathcal{L}^{\circ}(H)\right)\right)$ is an isomorphism.

Proof. The injectivity is clear from the following diagram:

$$
\begin{gathered}
F^{p}\left(h^{k}\right) \rightarrow H^{k}\left(X ; F^{p}\left(\mathcal{L}^{\circ}(H)\right)\right) \\
\bigcap_{h^{k} \underset{\rightarrow}{\rightarrow} H^{k}\left(X ; \mathcal{L}^{\circ}(H)\right) .}
\end{gathered}
$$

Let us prove the surjectivity. Let $u$ be an element of $\Gamma\left(X ; F^{p}\left(\mathcal{L}^{k}(H)\right)\right) \subset$ $L_{(2)}^{k}\left(X^{*} ; H\right)$ with $d u=0$. Then we can write

$$
u=H(u)+\Delta G u=H(u)+\Delta g \quad \text { with } \quad g=G u .
$$

Since $H$ and $G$ preserve the Hodge decomposition, we find both $H(u)$ and $g$ are in $F^{b}\left(\mathbb{L}_{(2)}^{k}\left(X^{*} ; H\right)\right)$.

Now, $d u=0$ implies $d \xi=0$, and hence we obtain

$$
u=H(u)+d d^{*} g .
$$

Since $d^{*} g$ belongs to $F^{p-1}\left(L_{(2)}^{k-1}\left(X^{*} ; H\right)\right)$, we can write $d^{*} g=v_{0}+v_{1}$ with $v_{0} \in$ $L_{(2)}^{p-1, q}\left(X^{*} ; H\right)$ and $v_{1} \in F^{p}\left(L_{(2)}^{k-1}\left(X^{*} ; H\right)\right)$ with $q=k-p$. Therefore we have 


$$
u=H(u)+d v_{0}+d v_{1}=H(u)+d^{\prime} v_{0}+d v_{1}+d^{\prime \prime} v_{0}
$$

at least in the distribution sense. Looking at the $(p-1, q+1)$-part, we obtain $d^{\prime \prime} v_{0}=0$, and, in particular, $v_{0} \in D\left(d^{\prime \prime}\right)$. Hence, by (6.2.8). we find

$$
v_{0}=H\left(v_{0}\right)+2 d^{\prime \prime} d^{\prime \prime *} G\left(v_{0}\right) .
$$

Thus we obtain, in the distribution sense,

$$
\begin{aligned}
u & =H(u)+2 d d^{\prime \prime} d^{\prime \prime *} G\left(v_{0}\right)+d v_{1} \\
& =H(u)+d\left(-2 d^{\prime} d^{\prime \prime *} G\left(v_{0}\right)+v_{1}\right) .
\end{aligned}
$$

Since $G\left(v_{0}\right) \in D(\Delta), d^{\prime \prime *} G\left(v_{0}\right) \in D\left(d^{\prime}\right)$ by Lemma 6.2.1, that is, $d^{\prime} d^{\prime \prime *} G\left(v_{0}\right)$ is in $L^{2}$. Since $-d^{\prime} d^{\prime \prime *} G\left(v_{0}\right) \in L_{(2)}^{p, q-1}\left(X^{*} ; H\right), v_{1} \in F^{p}\left(L_{(2)}^{k-1}\left(X^{*} ; H\right)\right)$ and $H(u) \in F^{p}\left(h^{k}\right)$, we are done.

Thus we obtain

Theorem 6.2.3。 (i) $\alpha: \quad H^{k}\left(X ; F^{p}\left(\mathcal{L}^{\circ}(H)\right) \rightarrow H^{k}\left(X ; \mathcal{L}^{\circ}(H)\right)\right.$ and

$$
\beta: \quad H^{k}\left(X ; \bar{F}^{q}\left(\mathcal{L}^{\circ}(H)\right) \rightarrow H^{k}\left(X ; \mathcal{L}^{\circ}(H)\right)\right.
$$

are injective.

(ii) Let us denote by $F$ and $\bar{F}$ the filtrations of $H^{k}\left(X ; \mathcal{L}^{\circ}(H)\right) \cong H^{k}\left(X ;{ }^{\pi} H\right)$ given by $\alpha$ and $\beta$ in (i), respectively. Then $H^{k}\left(X ;{ }^{\pi} H\right)$ has a pure Hodge structure of weight $k+w$.

Note that $\mathcal{L}^{\circ}(H), F\left(\mathcal{L}^{\circ}(H)\right)$ and $\bar{F}\left(\mathcal{L}^{\circ}(H)\right)$ do not depend on the choice of a Kähler metric, therefore neither does the Hodge structure on $H^{k}\left(X ;{ }^{\pi} H\right)$.

$\S 6.3 . \quad$ Let us define

$$
\mu_{0}:\left(\mathcal{E}_{X}^{p} \otimes H_{C}\right) \otimes\left(\mathcal{E}_{X}^{p^{\prime}} \otimes \bar{H}_{C}\right) \rightarrow \mathcal{E}_{X}^{p+p^{\prime}}
$$

by

$$
\mu_{0}((\alpha \otimes e) \otimes(\beta \otimes f))=S(e, f) \alpha \wedge \beta
$$

Then we have the following

Lemma 6.3.1. The map $\mu_{0}$ naturally extends to a map

$$
\mu: \mathcal{L}^{p}(H) \otimes \mathcal{L}^{p \prime}(\bar{H}) \rightarrow \mathscr{D} k_{X}^{p+p \prime},
$$

and the map $\mu$ commutes with the exterior differentiation $d$.

Proof. Let $x$ be a point in $Y=X \backslash X^{*}$ and let $U$ be an open neighborhood of $x$ in $X$. Since the product of two square-integrable functions is integrable, 
the image of the map obtained by extending $\mu_{0}$ to $\Gamma\left(U ; \mathcal{L}^{p}(\bar{H})\right) \otimes \Gamma\left(U ; \mathcal{L}^{p^{\prime}}\left(\mathbb{H}^{F}\right)\right)$ is contained in the space of integrable $\left(p+p^{\prime}\right)$-forms on $X^{*}$ with respect to the metric form $\omega$. Since any compactly supported $C^{\infty}$-form on $X$ has a finite supremum with respect to $\omega$, the image of the map st thus obtained is contained in $\mathscr{D} b_{X}^{p+p \prime}$.

To verify that the map $\&$ commutes with $d$, it suffices to show

$$
\int d u \wedge v \wedge \varphi+(-1)^{p} \int u \wedge d v \wedge \varphi+(-1)^{p+p^{\prime}} \int u \wedge v \wedge d \varphi=0
$$

holds for any $u$ in $\Gamma\left(U ; \mathcal{L}^{p}(\mathbb{H})\right)$, any $v$ in $\Gamma\left(U ; \mathcal{L}^{p^{\prime}}(\bar{H})\right)$ and each compactly supported $\left(2 n-p-p^{\prime}-1\right)$-form $\varphi$ on $U$. Multiplying a compactiy supported $C^{\infty}$-function which is equal to 1 ori a neighborhood of supp $\varphi$, we may assume from the firsi that both $u$ and $v$ are compartly supported in $U$. Since the weak closure of $d$ coincides with its strong closure, there exist sequences $u_{n}$ and $v_{n}$ of compactiy supported $C^{\infty}$-forms on $X^{*}$ such that $u_{n} \rightarrow u_{9} v_{n} \rightarrow v_{9} d u_{n} \rightarrow d u$ and $d v_{n} \rightarrow d v$ with respect to the $L^{2}$-norm. Since $d$ and $\mu_{0}$ commute by the flatness of $S,(6.3 .1)$ holds for $u_{n}$ and $v_{n}$ in place of $u$ and $v$, respectively. Hence we obtain $(6.3 .1)$ by taking the limit.

Q.E.D.

Let $\mathbb{C}_{1}$ denote the trivial extension to $\mathcal{E}^{\circ} \otimes \mathbb{R}^{\circ}$ of the Weil operator $\mathbb{C}(H)$, i.e., $C_{3}=\mathrm{id} \mathcal{C}^{\circ} \otimes C(H)$. Since the image of $\mu$ is contained in the space of the integrable (2n)-forms on $X^{*}$, we find

(6.3.2) $\mu\left(\mathrm{C}_{1} u \wedge * \bar{u}\right)$ is a non-negative measure for any $u$ in $L_{(2)}^{n-k}\left(X^{*} ; \mathbb{H}\right)$. (Cf. [W].)

$\S \Phi_{0} \Lambda_{0}$ Let $L$ be the operator $L_{(2)}^{k}\left(X^{*} ; H\right) \rightarrow L_{(2)}^{k+2}\left(X^{*} ; \mathbb{H}^{\prime}\right)$ given by the exterior multiplication of $\omega$. Then, since $\omega$ has finite length, $L$ is a continuous operator. Let $\mathbb{A}$ be the adjoint operator of $L$. Then as in [W], we have

$$
\left.[\mathbb{1}, \mathbb{L}]\right|_{L_{(2)}^{k}\left(X^{*} ; H\right)}=n-k \text {. }
$$

Thus we can apply the usual technique to show that

$$
\mathbb{L}^{k}: \mathbb{L}_{(2)}^{n-k}\left(\mathbb{M} * ; \mathbb{H}^{*}\right) \rightarrow \mathbb{L}_{(2)}^{n+k}\left(X^{*} ; \mathbb{H}^{T}\right)
$$

is bijective.

Since $[\Delta, \mathbb{L}]=0$, we have

$$
L^{k}: h^{n-k} \rightarrow h^{n+k} \text { is bijective . }
$$

Now let $l \in \mathbb{H}^{2}(X ; \mathbb{R})$ denote the cohomology class of $\omega_{0}$. Then we have 
the following

Lemma 6.4.1.

$$
H^{k}\left(X ;{ }_{\uparrow}^{\pi} H\right) \stackrel{l}{\rightarrow} H^{k+2}\left(X ;{ }^{\pi} H\right)
$$

is a commutative diagram.

Proof. Since every $\mathbb{C}^{\infty}$-form on $X$ has a finite length, we have the homomorphism of complexes

$$
\mathcal{E}^{\circ} \otimes \mathcal{L}^{\circ}(H) \rightarrow \mathcal{L}^{\circ}(H)
$$

Passing to the cohomology groups, we have

$$
H^{2}\left(X ; \mathcal{E}^{\circ}\right) \otimes H^{k}\left(X ; \mathcal{L}^{\circ}(H)\right) \rightarrow H^{k+2}\left(X ; \mathcal{L}^{\circ}(H)\right) \text {. }
$$

Therefore, for $\alpha \in h^{k}, l \alpha$ is represented by the closed $L_{2}$-form $\omega_{0} \wedge \alpha$. Hence it is enough to show that $\omega \wedge \alpha-\omega_{0} \wedge \alpha=i c \partial \bar{\partial} \varphi \wedge \alpha$ belongs to the $L^{2}$-coboundary. This is clear, because $\partial \bar{\partial} \varphi \wedge \alpha=d(\bar{\partial} \varphi \wedge \alpha)$ and $\bar{\partial} \varphi$ has also finite length.

Q.E.D.

Now we have the following

Theorem 6.4.2. (i) (Hard Lefschetz Theorem)

$$
l^{k}: H^{n-k}\left(X ;{ }^{\pi} H\right) \rightarrow H^{n+k}\left(X ;{ }^{\pi} H\right)
$$

is an isomorphism (of Hodge structures).

(ii) Let $P_{k}$ denote the kernel of

$$
l^{k+1}: H^{n-k}\left(X ;{ }^{\pi} H\right) \rightarrow H^{n+k+2}\left(X ;{ }^{\pi} H\right) .
$$

Then

$$
(-1)^{(n-k)(n-k-1) / 2}\left(\alpha, l^{k} \beta\right)
$$

gives a polarization of the Hodge structure on $P_{k}$. Here $($,$) is the pairing$ given by

$$
S:{ }^{\pi} H_{C} \otimes{ }^{\pi} \bar{H}_{C} \rightarrow \mathbb{C}_{X}
$$

and

$$
\begin{aligned}
& H^{n-k}\left(X ;{ }^{\pi} H_{C}\right) \otimes H^{n+k}\left(X ;{ }^{\pi} \bar{H}_{C}\right) \\
\rightarrow & H^{2 n}\left(X ;{ }^{\pi} H_{C} \otimes{ }^{\pi} \bar{H}_{C}\right) \stackrel{H^{2 n}(S)}{\longrightarrow} H^{2 n}(X ; \mathbb{C}) \rightarrow \mathbb{C} .
\end{aligned}
$$

Proof. (i) This is an immediate consequence of (6.4.2) and Lemma 6.4.1. (ii) For $\alpha$ and $\beta$ in $P_{k}$, let us choose harmonic representatives $f$ and $g$ of $\alpha$ 
and $\beta$, respectively. Let $\mathbb{C}_{0}$ denote the trivial extension to $\mathcal{E}^{\circ} \otimes \mathbb{H}_{\mathbb{C}}$ of the Weil operator on $\mathcal{E}^{\circ}$. (ci. Proposition 6.1.1. (i)) Then it follows from (6.2.4) that $\left.\mathbb{C}_{0} \mathbb{C}_{1}\right|_{n^{n-k}}$ coincides with $\mathbb{C}\left(\mathbb{H}^{n-k}\right)$, the vilu operator on $\mathbb{H}^{n-k}\left(\mathbb{X} ;{ }^{n z} \mathbb{H}\right)$. Hence we have

$$
\left(\mathbb{C}\left(\mathbb{H}^{n-k}\right) \alpha, l^{k} \bar{\beta}\right)=\int \mu\left(\mathbb{C}_{0} \mathbb{C}_{1} \rho \wedge L^{k} \bar{g}\right)
$$

On the other hand, Proposition 6.1.1 (i) tells us

$$
* \mathbb{C}_{0} f=(-1)^{(n-k)(n-k-1) / 2} \frac{1}{k !} \mathbb{L}^{k} f \text {, }
$$

if $L^{k+1} f=0$. Therefore, if $\alpha^{\prime}=\beta$ (and hence we choose $f=g$ ), we find

$$
\left(\mathbb{C}\left(\mathbb{H}^{n-k}\right) \alpha^{\prime} l^{k} \bar{\alpha}\right)=k !(-1)^{(n-k)(n-k-1) / 2} \int \mu\left(\mathbb{C}_{2} \mathbb{C}_{0} f \wedge * \mathbb{C}_{0} \bar{j}\right)
$$

Then the proof is completed by $(6.3 .2)$.

\section{Aupperadin: Review om Fumationall fundlysis}

In order to fix the notions and notations we hist up basic facts concerning Hillbert spaces and operators upon them. We follow [Ch], [H], [Ko] and [Y] in our presentation.

$\S \mathbb{A}_{0} \mathbb{1}_{\text {. Let }} H_{1}$ and $H_{2}$ be two Hilberi spaces. An operator $T$ from $Z_{1}$ to $I_{2}$ is, by definition, a $\mathbb{C}$-linear homomorphism from a. linear subspace $\mathbb{D}(\mathbb{T})$ of $H_{1}$ to $H_{2}$. We sometimes use the symbol $T: H_{1} \rightarrow H_{2}$ to denote an operator $T$ from $\mathbb{H}_{1}$ to $\mathbb{I}_{2}$, although $D(T)$ may not be $\mathbb{E}_{1}$. The linear space $\mathbb{D}(T)$ is called the domain of $T$. If $D(T)$ is dense in $H_{1}$, we say $T$ is densely defined. The graph $G(T)$ of an operator $T$ is, by definition, $\left\{(x, T x) \in \mathbb{H}_{1} \times H_{2} ; x \in \mathbb{D}(\mathbb{T})\right\}$. We denote by $G^{a}(\mathbb{T})$ the space $\left\{(-\mathbb{T} x, x) \in H_{2} \times \mathbb{H}_{1} ; x \in \mathbb{D}(\mathbb{T})\right\}$. If $G(\mathbb{T})$ is closed, we call $T$ a closed operator. Note that, even for a densely defined closed operator $\mathbb{I}$, there may exist another closed operator $F_{i}^{\prime \prime}$ such that $\mathbb{D}\left(\mathbb{T}^{\prime}\right)$ $\supsetneq \mathbb{D}(T)$ and $T x^{\prime}=\mathbb{T} x$ for $x \in \mathbb{D}(T)$, if $D(T) \neq \mathbb{H}_{I}$. In what follows, $R(\mathbb{T})$ denotes the range of $\mathbb{T}$, i.e., $\{\mathbb{T} x ; x \in D(\mathbb{T})\}$ and $\mathbb{K}$ er $\mathbb{T}$ denotes $\{x \in D(T) ; \mathbb{T} x=0\}$. [As we frequently use the symbol $N$ in this paper, we avoid the more common notation $N(T)$.] For a densely defined operator $T$, we define its adjoint operator $T^{*}$ from $H_{2}$ to $I_{1}$ by $G\left(T^{*}\right)=G^{a}(T)^{\perp}$, the orthogonal complement of $G^{a}(T)$; in other words,

$$
G\left(\mathbb{T}^{*}\right)=\left\{(x, y) \in H_{2} \times H_{1} ;(x, T z)_{H_{2}}=(y, z)_{H_{1}} \text { for any } z \text { in } D(T)\right\} .
$$


Then $T^{*}$ is a closed operator, and, if $T$ is a densely defined closed operator, then $T^{*}$ is also densely defined and $T^{* *}=T$ holds.

The pre-Hilbert structure of $D(T)$ induced by $D(T) \rightarrow G(T) \subset H_{1} \oplus H_{2}$ is called the graph norm. If $T$ is a closed operator, then $D(T)$ is a Hilbert space with the graph norm.

Lemman $\mathbb{A}_{0} \mathbb{1}_{0}$. Let $T$ be a densely defined closed operator and $E$ a dense subset of $D(T)$ (in the norm of $H_{1}$ ). If $\left(\left.T\right|_{E}\right)^{*}=T^{*}$, then $E$ is dense in $D(T)$ with respect to the graph norm. Here $\left.T\right|_{E}$ denotes the restriction of $T$ to $E$ with $D\left(\left.T\right|_{E}\right)=E$.

In fact, $\left(\left.T\right|_{E}\right)^{*}=\mathbb{T}^{*}$ means $G\left(\left.T\right|_{E}{ }^{\perp}\right)=G\left(T^{\perp}\right)$. Hence $G\left(\left.T\right|_{E}\right)$ is dense in $G(T)$.

The following proposition is usually referreci to as the closed range theorem (of Banach).

Proposition A.1.2. Let $T$ be a densely defined closed operctor. If $R(T)$ is closed, so is $R\left(T^{*}\right)$ and $R(T)=\left(\operatorname{Ker} T^{*}\right)^{\perp}$ holds.

See e.g. [Y], p. 205 for the proof.

The importance of a closed operator with closed range lies in the following

Proposicion A.1.3. (The open mapping theorem) If $T: \mathrm{H}_{1} \rightarrow \mathrm{H}_{2}$ is a closed operator with closed range, then $T$ is an open mapping and $\left(T \mid(\operatorname{Ker} T)^{\perp} \cap D(T)\right)^{-1}$ is a bounded operator from $\mathbb{R}(T)$ to $(\operatorname{Ker} T)^{\perp}$.

See e.g. [Y], p. 79 for the proof.

$\S \mathbb{A}_{0}$ 2。 Let $H_{1}, H_{2}$ and $H_{3}$ be three Hilbert spaces. Let $T: H_{1} \rightarrow H_{2}$ and $S: H_{2} \rightarrow H_{3}$ be densely defined closed operators. We assume

$$
R(T) \subset D(S)
$$

and

$$
S T x=0 \quad \text { for } \quad x \in D(T) .
$$

Then $S^{*}: H_{3} \rightarrow H_{2}$ and $T^{*}: H_{2} \rightarrow H_{1}$ satisfy the same conditions, namely,

$$
R\left(S^{*}\right) \subset D\left(T^{*}\right)
$$

and

$$
T^{*} S^{*} x=0 \quad \text { for } \quad x \in D\left(S^{*}\right)
$$


We define $\Delta: H_{2} \rightarrow H_{2}$ by

$$
\begin{aligned}
& D(\Delta)=\left\{x \in D(S) \cap D\left(T^{*}\right) ; T^{*} x \in D(T), S x \in D\left(S^{*}\right)\right\} \\
& \Delta x=S^{*} S x+T T^{*} x \quad \text { for } \quad x \in D(\Delta) .
\end{aligned}
$$

\section{Lemmma $\mathbb{A}_{0} 2_{0} \mathbb{H}_{\circ} \Delta$ is a closed operator.}

Proof. Assume that a sequence $x_{n} \in D(\Delta)$ converges to $x$ and $\Delta x_{n}$ converges to $y$. We shall show $x \in D(\Delta)$ and $\Delta x=y$. Setting $z=x_{n}-x_{m}$, we have

$$
(\Delta z, z)_{H_{2}}=\left(T^{*} z, T^{*} z\right)_{H_{1}}+(\mathbb{S} z, S z)_{H_{2}} \text {. }
$$

Therefore $T^{*}\left(x_{n}-x_{m}\right)$ and $S\left(x_{n}-x_{m}\right)$ tends to zero and hence $\mathbb{T}^{*} x_{n}$ and $S x_{n}$ converge. This implies $x \in D(S) \cap D\left(T^{*}\right)$ and $T^{*} x_{n}$ (resp., $S x_{n 2}$ ) converges to $\mathbb{T}^{*} x$ (resp., Sy). Since $(\Delta z, \Delta z)_{H_{2}}=\left(S^{*} S z, S^{*} S z\right)_{H_{2}}+\left(\mathbb{T} T^{*} z, \mathbb{T} T^{*} z\right)_{H_{2}}, \mathbb{S}^{*} \mathbb{S} x_{n}$ and $T^{*} T x_{n}$ converge. Thus we find $S x \in D\left(S^{*}\right), \mathbb{T} x \in \mathbb{D}\left(T^{*}\right)$, and $\mathbb{S}^{*} S x_{n}$ (resp., $T^{*} T x_{n}$ ) converges to $S^{*} S x$ (resp., $T^{*} T x$ ). Hence $x \in D(\Delta)$ and $\Delta x=y$.

Now we have the following abstract version of the Hodge-Kodaira decomposition due to $\mathbb{K}$ omatsu [Ko].

Theorem A.2.2。 Let $S, T$ and $\Delta$ be as above, and suppose that both $\mathbb{R}(T)$ and $R(S)$ are closed. Denoting $\operatorname{Ker} \Delta$ by h, we find ihe following:

(i) $\Delta$ is self-adjoint, that is, $\Delta=\Delta^{*}$ holds.

(ii) $h=\mathbb{K} \operatorname{er}\left(T^{*}\right) \cap \operatorname{Ker} S$ and $h^{\perp}=R(\Delta)$.

(iii) Denoting by $p_{h}$ the projection operaior: $H_{2} \rightarrow h$, we find that the operator $\left(\left.\Delta\right|_{h^{\perp}}\right)^{-1}\left(1-p_{h}\right)$ is well-defined and bounded.

Defumition A.2.3. The operator $\left(\left.\Delta\right|_{h \perp}\right)^{-1}\left(1-p_{h}\right)$ is called the Green operator.

As the reference [ $\left.\mathbb{K o}_{\mathrm{o}}\right]$ is written in Japanese, we give the proof of the theorem for the convenience of the reader. It is exactly the same as [Ko] p. 124 p. 125.

Let us first verify (ii). Since $h=\mathbb{K} \operatorname{er}\left(\mathbb{T}^{*}\right) \cap \mathbb{K}$ er $\mathcal{S}$ is clear, it suffices to verify

$$
\left(\operatorname{Ker}\left(\mathbb{T}^{*}\right) \cap \mathbb{K e r} S\right)^{\perp}=\mathbb{R}(\Delta)
$$

Since $S$ is supposed to be a closed operator with closed range, $\mathbb{R}\left(S^{*}\right)=$ $(\operatorname{Ker} S)^{\perp}$ holds. Hence we find

$$
h \oplus \mathbb{R}\left(S^{*}\right)=\mathbb{K} \operatorname{er}\left(T^{*}\right)
$$


Here and in what follows, the symbol $A \oplus B$ means that $A$ and $B$ are closed subspaces which are mutually orthogonal. Since $T$ is supposed to be of closed range, we find

$$
H_{2}=\mathbb{K e r}\left(T^{*}\right) \oplus \mathbb{R}(T)
$$

Combining (A.2.8) and (A.2.9), we find

$$
H_{2}=R(T) \oplus h \oplus R\left(S^{*}\right)
$$

In particular,

$$
R(\Delta) \subset h^{\perp} \text {. }
$$

Let us now verify $h^{\perp} \subset \mathbb{R}(\Delta)$. Let $\widetilde{\mathbb{T}}$ denote $\left.T\right|_{(\operatorname{Ker} T)^{\perp}}{ }_{n D(T)}$, i.e. $\widetilde{\mathbb{T}}$ is a map from $(\operatorname{Ker} T)^{\perp} \cap D(T)$ to $R(T)$. It then follows from the open mapping theorem that $\widetilde{T}$ has a bounded inverse on $R(T)$. Since $R\left(T^{*}\right)$ is also closed by the closed range theorem, $\widetilde{T}^{*}=T^{*}\left(\operatorname{Ker} T^{*}\right)^{\perp} \cap D\left(T^{*}\right)$ has also bounded inverse on $R\left(T^{*}\right)=(\operatorname{Ker} T)^{\perp}$. Hence, for $y$ in $R(T), z=\left(\widetilde{T}^{*}\right)^{-1}(\widetilde{T})^{-1} y=G_{\text {def }} y$ is welldefined, and $G_{1}$ is a bounded operator. We have $T T^{*} G_{1} y=y$ for $y \in R(T)$ such that $\mathbb{S}^{*} S G_{2} y=y$ for $y \in R\left(S^{*}\right)$. Similarly, as $R(S)$ and $R\left(S^{*}\right)$ are also closed by the assumption, we can find a bounded operator $G_{2}: R(S) \rightarrow R(S)$. Since $h^{\perp}=R(T) \oplus R\left(S^{*}\right)$ follows from (A.2.10), and since $R(T) \subset \mathbb{K e r} S$ and $R\left(S^{*}\right) \subset \mathbb{K} e r T^{*}$ hold by the assumption, for each $y$ in $\iota^{\perp}$, we can find $z_{1} \in R(T)$ and $z_{2} \in \mathbb{R}\left(\mathbb{S}^{*}\right)$ so that

$$
y=T T^{*} z_{1}+S^{*} S z_{2}=\left(\mathbb{T} \mathbb{T}^{*}+S^{*} S\right)\left(z_{1}+z_{2}\right)
$$

holds. Thus we have verified $h^{\perp}=R(\Delta)$. The above reasoning also implies the boundedness of the operator $\left(\left.\Delta\right|_{h^{\perp}}\right)^{-1}\left(1-p_{h}\right)$. This completes the proof of (ii) and (iii).

If we set $\tilde{\Delta}=\left(\left.\Delta\right|_{h^{\perp}}\right)$, then $\tilde{\Delta}$ is clearly symmetric (i.e. $(\tilde{\Delta} u, v)=(u, \tilde{\Delta} v)$ for every $u, v \in \mathbb{D}(\tilde{\Delta})$ ). Furthermore it is invertible on $h^{\perp}$ as we have verified. Therefore it is self-adjoint on $h^{\perp}$. (See e.g. [Y], p. 199.) Since

$$
\Delta=\tilde{\Delta} p_{h^{\perp}}=p_{h^{\perp}} \tilde{\Delta} p_{h^{\perp}}
$$

hold, $\Delta$ is also self-adjoint. This completes the proof of (i).

Q.E.D.

$\S$ A.3. Let $M$ be a $C^{\infty}$-manifold with volume element $g$, and $V$ a $C^{\infty}$-vector bundle equipped with Hermitian form. Let us denote by $\mathbb{C}^{\infty}(M ; V)$ the space of $C^{\infty}$-sections of $V$, and by $C_{0}^{\infty}(M ; V)$ the space of compactly supported 
$\mathbb{C}^{\infty}$-sections of $\mathbb{V}$. Let us denote by $L_{(2)}(M ; V)$ the space of square integrable sections of $V$. Now, let $V_{1}$ and $V_{2}$ be two $C^{\infty}$-vector bundles and $P: C^{\infty}\left(M ; V_{1}\right)$ $\rightarrow C^{\infty}\left(M ; V_{2}\right)$ be a linear differential operator. Let $P^{*}: C^{\infty}\left(M ; V_{2}\right) \rightarrow C^{\infty}\left(M ; V_{1}\right)$ be its formal adjoint; i.e., the differential operator satisfying

$$
\begin{aligned}
& \left\langle P^{*} u \mid v\right\rangle=\left\langle\langle u \mid P v\rangle \quad \text { for } u \in \mathbb{C}^{\infty}\left(M ; V_{2}\right)\right. \text { and } \\
& v \in \mathbb{C}_{0}^{\infty}\left(M ; V_{1}\right) \text {. }
\end{aligned}
$$

The strong extension $P_{s}$ of $P$ is a linear operator: $L_{(2)}\left(M ; V_{1}\right) \rightarrow L_{(2)}\left(M ; V_{2}\right)$ such that $G\left(\mathbb{P}_{s}\right)$ is the closure of $G\left(\left.\mathbb{P}\right|_{C_{0}^{\infty}\left(M ; V_{3}\right)}\right)$. The weak extension $\mathbb{P}_{w}$ of $\mathbb{P}$ is, by definition, a linear operator given by

$$
P_{w}=\left(\left.P^{*}\right|_{C_{0}^{\infty}\left(M ; V_{2}\right.}\right) *,
$$

that is,

$$
D\left(P_{w}\right)=\left\{u \in L_{(2)}\left(M ; V_{1}\right) ; \text { the distribution } P u\right.
$$$$
\text { belongs to } \left.L_{(2)}\left(M ; \mathbb{V}_{2}\right)\right\} \text {. }
$$

Hence we find

$$
P_{w}=\left(\left(P^{*}\right)_{s}\right)^{*}, \quad P_{s}=\left(\left(P^{*}\right)_{w}\right)^{*} \text { 。 }
$$

This fact combined with Lemma A.1.1 entails the following

Lenmma A.3.1. The following conditions are mutually equivalent:

(i) $\quad \mathbb{P}_{s}=\mathbb{P}_{w}$

(ii) $C_{0}^{\infty}\left(M ; V_{1}\right)$ is dense in $D\left(P_{w}\right)$ with respect to the graph norm.

(iii) $\left(P^{*}\right)_{s}=\left(P^{*}\right)_{w}$

(iv) For $u \in D\left(P_{w}\right)$ and $v \in D\left(P^{*}\right)_{w}$, we have

$$
\left\langle\left\langle P_{w} u \mid v\right\rangle\right\rangle=\left\langle\left\langle u \mid\left(\mathbb{P}^{*}\right)_{w} v\right\rangle\right\rangle .
$$

Proof. (i) $\Leftrightarrow$ (ii) is obvious.

(ii) $\Leftrightarrow$ (iii) follows from Lemma A.1.1, because $\left(P^{*}\right)_{s}=\left(P_{w}\right)^{*}$ and $\left(P^{*}\right)_{w}=$

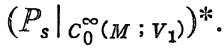

(i) $\Leftrightarrow$ (iv) is obvious.

$\S$ A.4. Let $M$ be a Riemannian manifold and let $H$ be a local system on $M$. Assume that the $C^{\infty}$-vector bundle associated with $H$ has a Hermitian metric. Let $L_{(2)}^{p}(M ; H)$ be the space of square integrable $H$-valued $p$-forms and let $C_{0}^{b}\left(M ; H^{T}\right)$ be the space of compactly supported $H$-valued $\mathbb{C}^{\infty}$-p -forms, and $C^{p}(M ; H)$ the space of $H$-valued $C^{\infty}$-p-forms. Then we can define the exterior derivative 


$$
d: C^{p}(M ; H) \rightarrow \mathbb{C}^{p+1}(M ; H)
$$

and its formal adjoint

$$
d^{*}: C^{p}(M ; H) \rightarrow C^{p-1}(M ; H)
$$

with respect to the inner product given by the Hermitian metric on $H$ and the Riemannian metric. The $p$-th $L_{2}$-cohomology group $H_{(2)}^{p}(M ; H)$ is also defined as the following quotient space:

(A.4.1) $\left\{u \in L_{(2)}^{p}(M ; H) ; d u=0\right.$ in the distribution sense $\}$ modulo

$\left\{d u ; u \in L_{(2)}^{p-1}(M ; H)\right.$ and the distribution $d u$, belongs to $\left.L_{(2)}^{p}(M ; H)\right\}$.

In order to compute the $L_{2}$-cohomology groups in terms of harmonic forms, i.e., forms annihilated by $\Delta=d d^{*}+d^{*} d$, we need to make precise the domain of the operators in question. Fortunately we have the following result due to Chernoff $[\mathrm{Ch}]$ :

Theorem A.4.1. Let $M$ be a complete Riemannian manifold, and let $H$ be a Hermitian vector bundle over $M$. Then $d_{s},\left(d^{*}\right)_{s},\left(d^{\prime}+d^{*}\right)_{s}$ and their powers are all essentially self-adjoint, i.e., their (operator-theoretic) adjoint is self-adjoint. In particular, $d_{s}=d_{w},\left(d^{*}\right)_{s}=\left(d^{*}\right)_{w},\left(d+d^{*}\right)_{s}=\left(d+d^{*}\right)_{w}$ and $\Delta_{s}=\Delta_{w}$ hold. Furthermore we find the following:

(A.4.2) $\left(d^{*}\right)_{w}$ is the (operator-theoretic) adjoint of $d_{w}$.

(A.4.3) $D\left(\Delta_{w}\right)=\left\{u \in D\left(d_{w}\right) \cap D\left(\left(d^{*}\right)_{w}\right) ; d_{w} u \in D\left(\left(d^{*}\right)_{w}\right),\left(d^{*}\right)_{w} w \in D\left(d_{w}\right)\right\}$.

Proof. Although [Ch], p. 410 discussed the case where $H$ is trivial, the argument there goes well without any change. In fact, since the symbol $\sigma(\xi)$ of $d$ is given by $\xi \wedge$, tensoring $H$ with the exterior bundle of cotangent bundle of $M$ has nothing to do with verifying the boundedness of $\| \sigma(\xi)|| /|\xi|$, which is needed there. The coincidence of the strong closure and the weak closure immediately follows from Lemma A.3.1 and the essential self-adjointness. Hence what remains to be proved is (A.4.3). It follows from the fact that the right hand side of (A.4.3) is closed with respect to the graph norm and the fact that $C_{0}(M ; H)$ is dense in $D\left(\Delta_{s}\right)=D\left(\Delta_{w}\right)$.

\section{Referemees}

[C-K, 1] Cattani, E.H. and Kaplan, A.G., The monodromy weight filtration for a several variables degeneration of Hodge structures of weight two, Inventiones math., 52 (1979), 131-142. 
[C-K, 2] — Polarized mixed Hodge structures and the local monodromy of a variation of Hodge structure, Inventiones math., 67 (1982), 101-115.

[C-K-S, 1] Cattani, E., Kaplan, A. and Schmid, W., Degeneration of Hodge structures, Ann. of Math., 123 (1986), 457-535.

$[\mathrm{C}-\mathrm{K}-\mathrm{S}, 2] \longrightarrow, L_{2}$ and iniersecton cohomoloies for a polarizable variaticn , $f$ Hodge structure, Invent. math., 87 (1987), 217-252.

[Ch] Chernoff, P.R., Essential self-adjointness of powers of generators of hyperbolic equations, J. Functional Analysis, 12 (1973), 4.01-414.

[D] Deligne, P., Théorie de Hodge, I, Actes, Congrès intern Math., $\mathbb{1}$, pp. 425430, Gauthier-Villars, Paris, 1971; II and III, Publ. Math. IHES, \&0 (1971), 5-57 and $\Delta 4$ (1974), 5-78.

[G] Godement, R.. Theórie des faisceaux, Fermann, Paris, 1964.

[G-M] Goresky, M. and MacPherson. R., Iniersection homology II, Inventiones math., 72 (1983), 77-129.

[G-S] Griffichs, P. and Schmid, W., Locally homogeneous complex manifolds, Acta Math., 123 (1969), 253-302.

[H-L-P] Hardy, G.H.. Littlewood, J.E. and Pólya, G., Inequalities, Cambridge Univ. Press, Cambridge, 1934.

[H] Hörmander, L., $L^{2}$-estimates and existence theorems for the $\bar{\partial}$ operator, Acta Math., 113 (1965), 89-152.

[K] Kashiwara, M., The asymptotic behavior of a variation of polarized Hodge structure, Pucl. RIMS, Kyoto Univ., $2 \mathbb{1}$ (1985), 853-875.

[K-K, 1] Kashiwara, M. and Kawai, T., The Poincaré lemma for a variation of polarized Hodge structure, Prcc. Japan Acad., 61 (1985), 164-167.

[K-K, 2] Hodge structure and holonomic systems, Proc. Japan Acad., 62 (1986), 1-4.

[Ko] Komatsu, H., The Theory of Hyperfunctions and Linear Partial Differenial Equations, Univ. of Tokyo, Tokyo. 1968, in Japanese.

[S] Schmid, W., Variation of Hodge structure: the singularities of the period mapping, Inventioes math., 22 (1973), 211-319.

[Se] Serre, J.-P., Géométrie algébrique et géométrie analytique, Ann. Inst. Fourier, 6 (1956), 1-42.

[W] Weil, A., Introduction à l'éiude des variétés kähıoriennes, Hermann, Paris, 1958.

[Y] Yosida, K., Funrtional Analysis, Springer, Berlin-Göttingen-Heidelberg, 1965.

[Z] Zucker, S., Hcdge theory with degenerating coefficients: $L_{2}$ cohomology in the Poincaré metric, Ann. of Math., 109 (1979), 415-476. 
